\title{
Personalization of Wearable-Based Exergames with Continuous Player Modeling
}

by

Zhao Zhao

A thesis submitted to the Faculty of Graduate and Postdoctoral Affairs in partial fulfillment of the requirements for the degree of

\author{
Doctor of Philosophy \\ in
}

Electrical and Computer Engineering

Carleton University

Ottawa, Ontario

(C) 2019, Zhao Zhao 


\begin{abstract}
In recent years, sedentary behavior has been recognized as a major lifestyle-related health risk. Meanwhile, exergames and gamification of physical activities are effective tools to motivate behavior change, particularly to promote daily physical activities. Research has shown that persuasive technologies and gamification can be utilized to increase physical activity. On the other hand, studies have suggested that a "one-size-fitsall" approach does not work well for persuasive game design. At the same time, player modeling and recommender systems are increasingly used for personalizing contents and services.
\end{abstract}

However, there is limited existing work on how to build comprehensive player models for personalizing gamified systems and recommending daily physical activities, and on the long-term effectiveness of gamified exercise-promoting systems. To fill these gaps, a new approach for gamified 24/7 fitness recommendation systems is introduced in this research. It uses wearable activity trackers combined with continuous player modeling to provide personalized activity recommendations and generates gamified content targeted to each user.

Preliminary results show the feasibility of using wearable activity trackers for gamification of physical activities, and the effectiveness of using player modeling for generating personalized exercise recommendations. We show that personalizing recommendations using player modeling and gamification with wearables will improve users' engagement and motivation towards fitness activities over time. 


\section{Acknowledgements}

I would like to express my special appreciation and thanks to my advisor Professor Dr. Ali Arya, you have been a tremendous mentor for me. Your advice on both research as well as on my career have been invaluable.

I am grateful to all of those with whom I have had the pleasure to work during this and other related projects. My sincere gratitude to Dr. Ali Etemad, Dr. Rita Orji and Gerry Chan, for their willingness to help me with my research during all these years.

I would like to offer special thanks to Dr. Anthony Whitehead, although no longer with us, his decency, sincerity, and kind soul will stay with us forever.

I would like to thank my family for all their love and encouragement. For my parents who supported me in all my pursuits. And for my loving, supportive, encouraging, and patient husband Howard whose faithful support during all stages of this Ph.D. is so appreciated. Thank you.

I also dedicate this thesis to my lovely daughter Raven, without whom this thesis would have been completed a year earlier. You are the pride and joy of my life. I love you more than anything. 


\section{Table of Contents}

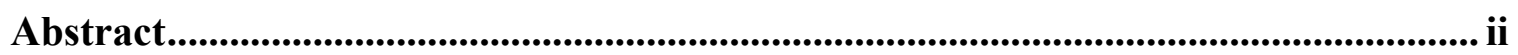

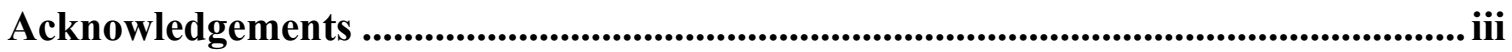

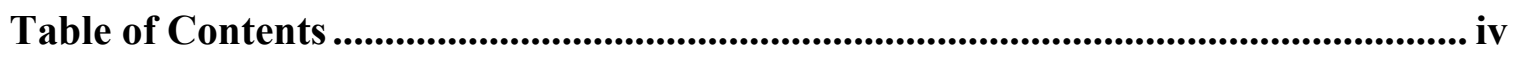

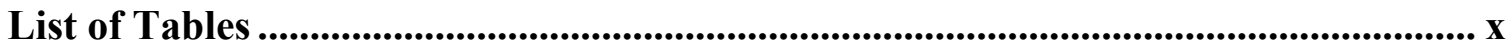

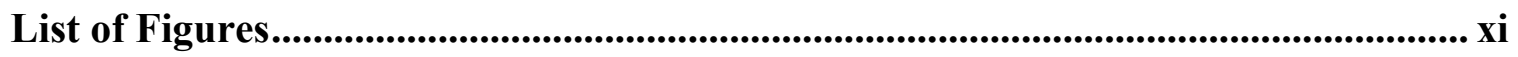

List of Appendices.................................................................................................................... xiv

List of Abbreviation ................................................................................................................. Xv

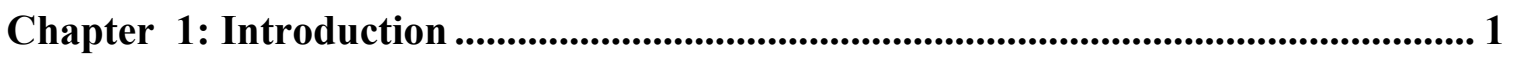

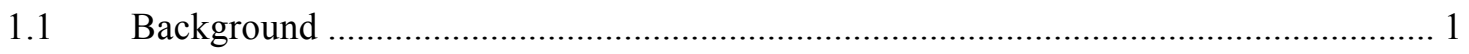

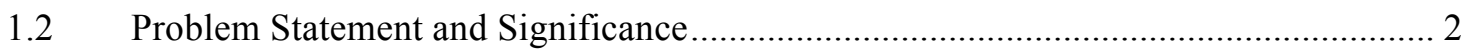

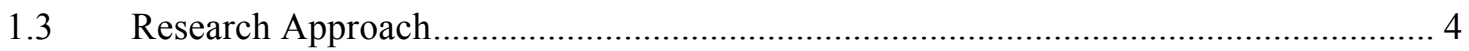

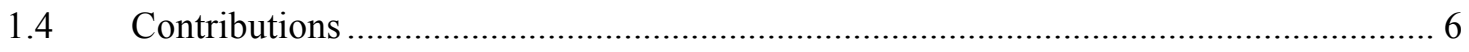

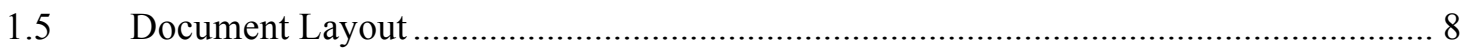

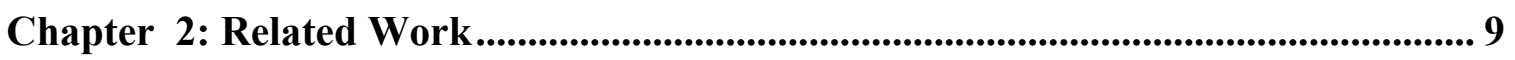

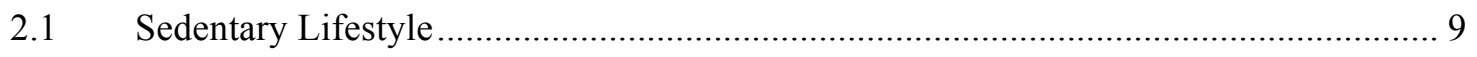

2.2 Exergames and Gamified Physical Activities ...................................................... 10

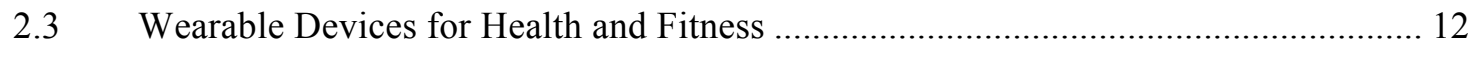


2.4 Player Models

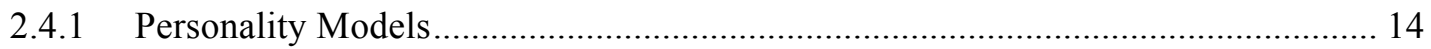

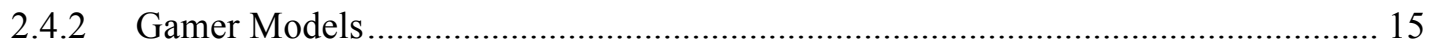

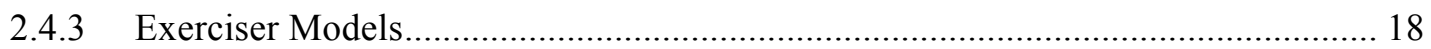

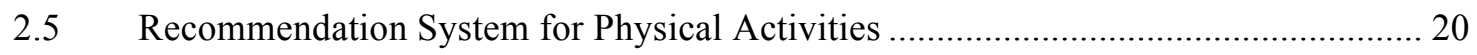

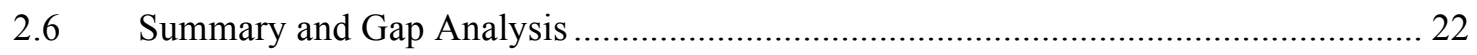

Chapter 3: Proposed Theoretical Model, Solution, and Research .......................... 25

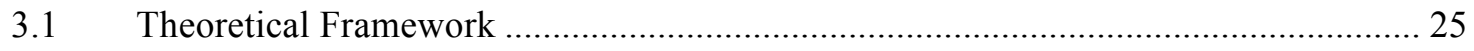

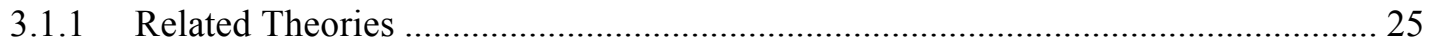

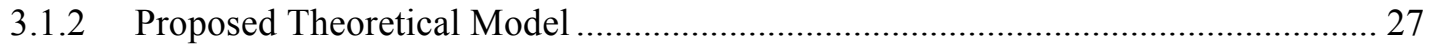

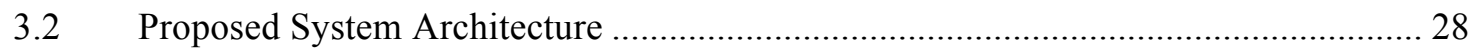

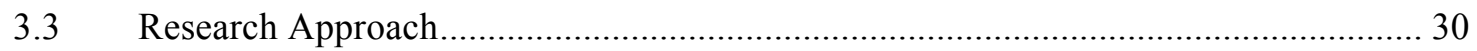

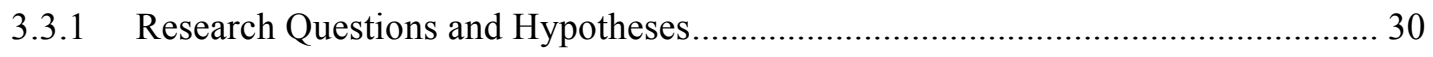

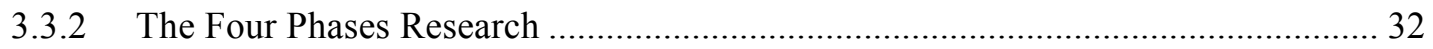

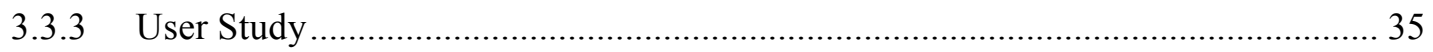

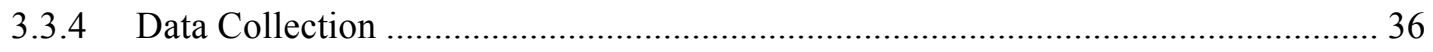

Chapter 4: Wearable-based Exergame (Research Phases 1 and 2) ......................... 37

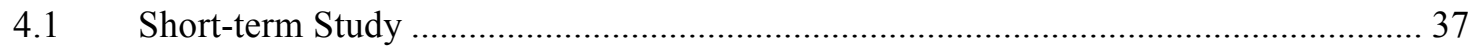


4.1.1 Overview

4.1.2 Application Design and Implementation .....

4.1.3 User Study Design

4.1.4 Result and Analysis.......

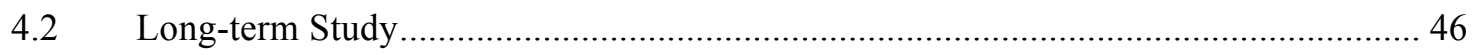

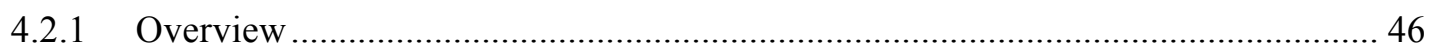

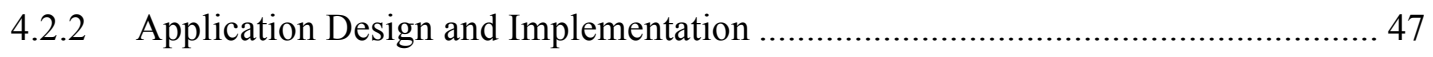

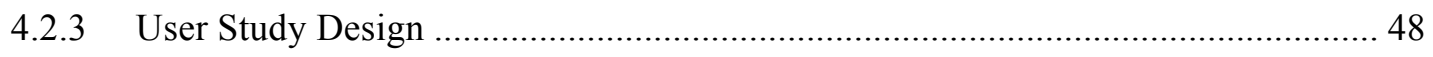

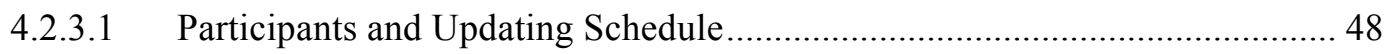

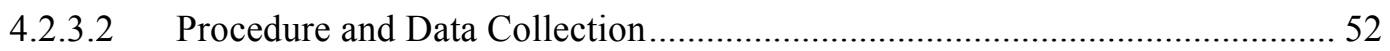

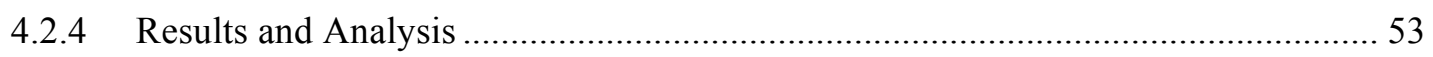

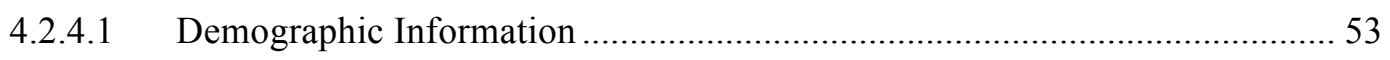

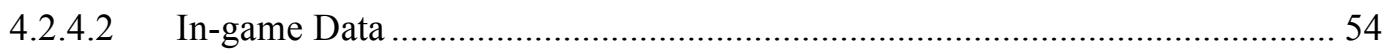

4.2.4.3 Overall Motivation, Satisfaction and Preference ......................................... 54

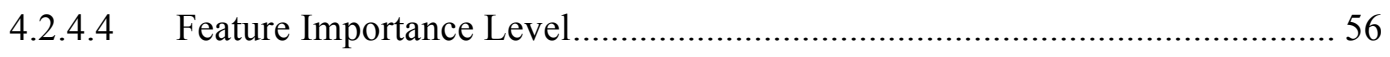

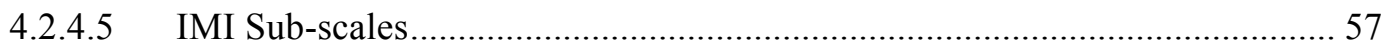

4.2.4.6 Daily Active Users and Workout Sessions................................................ 58 
5.1.1 Overview 64

5.1.2 Methodology 67

5.1.2.1 System Design 67

5.1.2.2 Application Design and Implementation 69

5.1.3 User Study Design 72

5.1.3.1 Participants and Group 72

5.1.3.2 Procedure and Data Collection. 75

5.1.4 Result and Analysis 76

5.1.4.1 General Information 76

5.1.4.2 Case Studies 77

5.1.4.3 Overall Motivation and Satisfaction

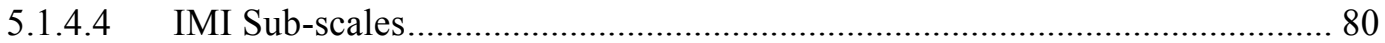

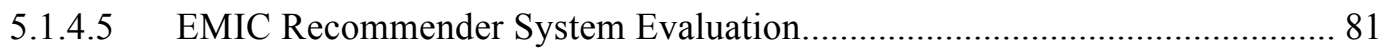

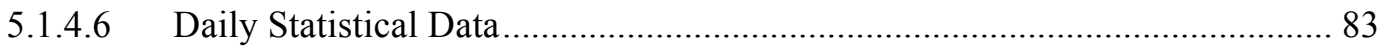

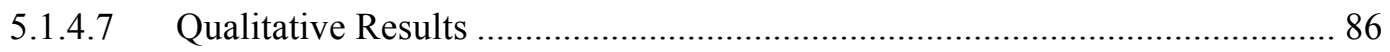


5.2.1 Overview

5.2.2 Survey

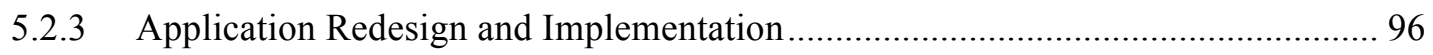

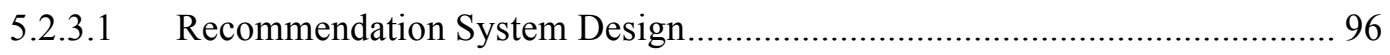

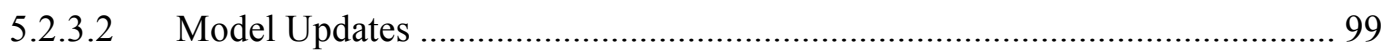

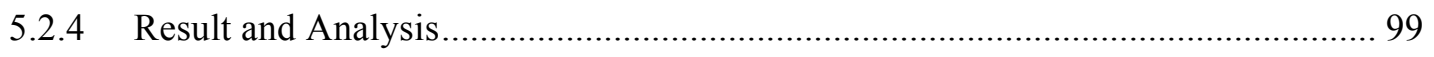

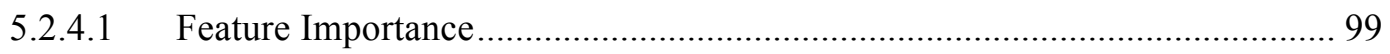

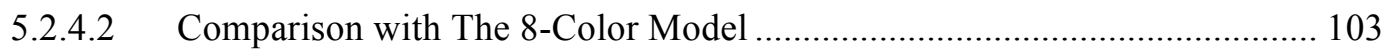

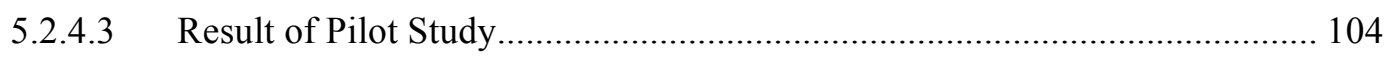

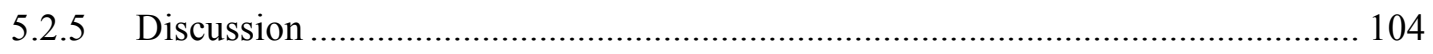

Chapter 6: Conclusion ......................................................................................................... 107

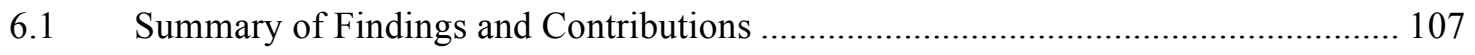

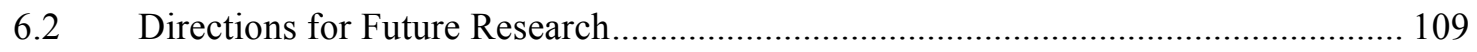

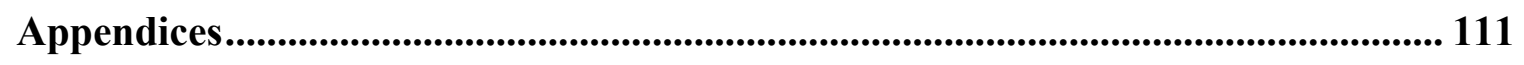

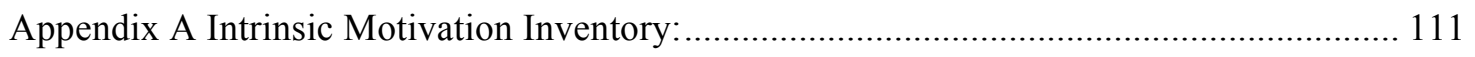


Appendix B EMIC (European Microsoft Innovation Center) recommender system evaluation measurement:

Appendix $\mathrm{C}$ The 8 color of fitness activity suggestions

Appendix D Survey items for research phase 4

References ....................................................................................................................... 122 


\section{List of Tables}

Table 3.1: Mapping of Research Questions to Research Phases .................................... 33

Table 4.1: Combination of Exercises and Wearable Devices...................................... 40

Table 4.2: List of Features Added in the New Application ......................................... 48

Table 4.3: List of Different Features.......................................................................... 51

Table 4.4: Automatic Updates Schedule used for the Updating Group........................... 51

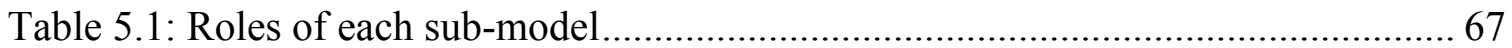

Table 5.2: Different game elements added for each type of player ............................. 71

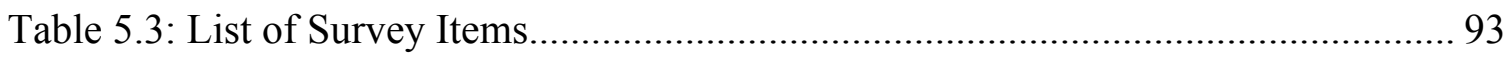

Table 5.4: Accuracy for the Binary Classification Prediction Model............................ 100

Table 5.5: Average feature importance score for all model parameters ....................... 101

Table 5.6: Example Comparison of Predictive Results between the 8 Color Model and our Prediction Model for User \#97 .......................................................................... 103 


\section{List of Figures}

Figure 3.1: Theoretical Model 28

Figure 3.2: Proposed System Architecture 29

Figure 3.3 Proposed Research Questions 31

Figure 4.1: Screenshots from Our Application. From left to right: choice of exercises, game view, and result view

Figure 4.2: Three Types of Exercises in Our User Study: running, cycling, and rope skipping 40

Figure 4.3: User Enjoyment for Different Modes of the Game 42

Figure 4.4: Amount of Fatigue for Different Modes of the Game 42

Figure 4.5: Sense of Accomplishment for Different Modes of the Games 43

Figure 4.6: Motivation for More Exercise based on Different Modes of the Games 44

Figure 4.7: Usefulness of the Application based on Different Modes of the Games 44

Figure 4.8: Overall Satisfaction of the Different Modes 46

Figure 4.9: Preference Scores for Different Combinations of Exercise and Device 46

Figure 4.10: Screenshots from Our Original Application. From left to right, row one: choice of player mode, choice of exercises, result screen, achievement screen; row two: landing screen, level 1 view, level 2 view, and level 3 view. 50 
Figure 4.11: Screenshots from Our Updated Application. From left to right: customized bird color for level1, customized background color for level 2, color picker panel view. 50

Figure 4.12: The Wearable Device used in Our Study (TI Sensortag) ........................... 51

Figure 4.13: Choice of Exercise (from in-game data monitoring)................................ 55

Figure 4.14: Level of Encouragement (asked after each completed workout session) .... 55

Figure 4.15: Result for Post-study Question 1, 2 and 3. From left to right: overall motivation, overall satisfaction, and overall preference over regular exercise. 56

Figure 4.16: Impact of Different Features for the Full and the Updating Groups ............ 57

Figure 4.17: Average and Standard Deviation of IMI: Interest/Enjoyment, Perceived Competence, Effort/Importance, Pressure/Tension, and Value/Usefulness. .................... 58

Figure 5.1: System architecture

Figure 5.2: Example of application interface and icon. Left: Android UI, right: Wear OS UI 70

Figure 5.3: Example of game elements. From left to right: profile in daily view (including points and challenges), profile in weekly view (including points and challenges), connect to Facebook view, hacker mode view, theme color customization view. 73

Figure 5.4: Example of recommendations for different groups. From left to right: Full, Personalized, Gamified, Control 73 
Figure 5.5: Results for post-study questions 1, 2 and 3. From left to right: overall motivation, overall satisfaction, and overall preference over regular exercise.

Figure 5.6: Average and Standard deviation of IMI: Interest/Enjoyment, Perceived Competence, Effort/Importance, Pressure/Tension and Value/Usefulness. 83

Figure 5.7: Average and Standard deviation of EMIC: perceived recommendation quality, perceived system effectiveness, general trust in technology, system-specific privacy concern.

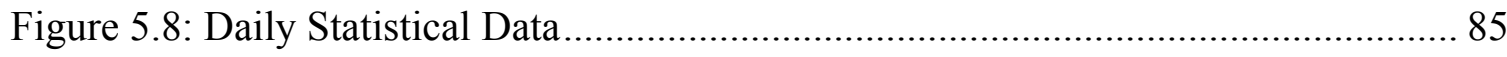

Figure 5.9: Number of Main Suggestions Received from Open-ended Questions 86

Figure 5.9: Overall Workflow of the Feature Importance Experiment in Azure. 94

Figure 5.10: Example of Feature Permutation Importance Scores (Basketball) 96

Figure 5.11: Overall Recommendation Flowchart 98

Figure 5.12: Average feature importance score for all model parameters (in in descending order) 102 


\section{List of Appendices}

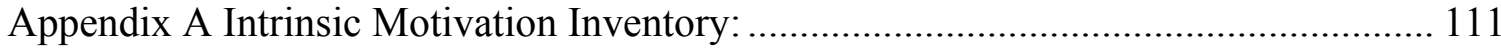

Appendix B EMIC (European Microsoft Innovation Center) recommender system

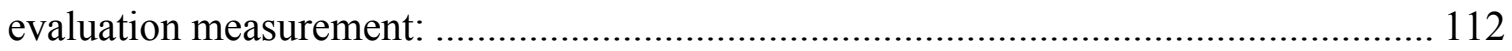

Appendix C The 8 color of fitness activity suggestions ............................................. 113

Appendix D Survey items for research phase 4..................................................... 114 


\section{List of Abbreviation}

\begin{tabular}{|c|c|}
\hline ANOVA & Analysis of Variances \\
\hline API & Application Programming Interface \\
\hline BMI & Body Mass Index \\
\hline CPAG & Canadian Physical Activity Guidelines \\
\hline DGD & Demographic Game Design model \\
\hline DGDB & Dynamic game difficulty balancing \\
\hline EMIC & European Microsoft Innovation Center \\
\hline GPS & Global Positioning System \\
\hline GRPAH & $\begin{array}{l}\text { Global Recommendations on Physical } \\
\text { Activity for Health }\end{array}$ \\
\hline HAR & Human Activity Recognition \\
\hline HSC & Health Status of Canadians \\
\hline IMI & Intrinsic Motivation Inventory \\
\hline MBTI & Myers-Briggs Type Indicator \\
\hline MUDs & Multi-User Dungeons \\
\hline OS & Operating System \\
\hline $\mathrm{PA}$ & Physical Activity \\
\hline RQ & Research Question \\
\hline RR & Relative Risk \\
\hline SVM & Support Vector Machine \\
\hline $\mathrm{TN}$ & True Negative \\
\hline TP & True Positive \\
\hline UI & User Interface \\
\hline VR & Virtual Reality \\
\hline WHO & World Health Organization \\
\hline WSS & Within-cluster Sum of Square \\
\hline
\end{tabular}




\section{Chapter 1: Introduction}

\subsection{Background}

A sedentary lifestyle is defined as a type of lifestyle where an individual does not receive regular amounts of physical activity (Lakka, 2003), and is becoming a significant public health issue (Wilmot, 2012). Nowadays, people are spending more and more time doing sedentary activities (Biddle, 2017). During leisure time, people are often sitting: while using a computer or other device, watching TV, or playing video games. Many of our jobs have become more sedentary, with long days sitting at a desk. Recent research is starting to confirm the health risks associated with a sedentary lifestyle (Tremblay, 2010).

Various solutions have been considered to encourage a more active lifestyle. For instance, a wide range of policies and programs were developed to promote more active lifestyles, such as to measure physical activity as a vital sign, or to create safer and healthier environments to walk or bike (Saelens, 2003). There were also communitywide campaigns focusing on using community resources to promote physical activity (Bauman, 2006). On the other hand, behavioral interventions have been shown to significantly increase physical activity resulting in improved control of diabetes and body mass index (Jakicic, 2005).

While combining exercise with gameplay has long been an effective approach for motivating more active lifestyles, the popularity of computer games has made it a more attractive option to combine digital games with exercise, introducing the new category of games called exergames (Consolvo, 2009). These games are strongly related to the notion 
of gamification (McGonial, 2011; Nicholson, 2015, Nacke and Deterding, 2017), which is the use of game concepts and methods in traditionally non-game applications ${ }^{1}$. Exergames have the potential of helping users achieve their fitness goals and increase engagement and pleasure by adding game features such as interactivity, multimedia content, various paces and challenges, and networking to physical activity. Considerable research has been devoted to the study of exergames from different perspectives, such as design, activity tracking, and gameplay mechanics (Sinclair, 2007). This research has resulted in significant progress in the design and development of exergames, but there are still many open research questions and problems. Some of these problems include the balance between enjoyment and intensity (Sinclair, 2007), the lack of standards in guiding the proper amount of exercise in-game (Perron, 2011), and how to use other technologies such as wearables and Virtual Reality (VR) to promote exergame experience (Tregillus, 2016), etc. Most of all, exergames suffer from retention problems, which has been an important and long-running investigation in the gaming industry (Whitehead, 2010).

\subsection{Problem Statement and Significance}

In order to have people maintain a constant habit or routine, applications should be designed with not only desirable usability, but also retention in mind, to provide users with sustainable motivation and engagement over time (Campbell, 2008). In digital games, user retention is a key metric for evaluating success. When it comes to exergames,

\footnotetext{
${ }^{1}$ Since exercise, in general, is not a game, exergames can be considered a type of gamification. On the other hand, we may limit gamification to cases such as using a leaderboard in a course where the activity is not a game.
} 
it is even more important to consider game sustainability since it takes time to form a habit of exercising.

Motivational affordances and gamification elements are deployed in exergaming in order to sustain behavior change and promote physical activity (Matallaoui, 2017). However, research also shows that existing approaches are not enough to sustain long-term motivation for exergames (Caro, 2018). Applications such as Pokémon Go, one of the most salient examples of an exergame, has reportedly reached tens of millions of worldwide. It was considered an effective means for motivating daily physical activity but still suffers from retention problems (Althoff, 2016).

Moreover, there were products bringing together wearable activity trackers and exergames or adding gamified elements to traditional activity tracking applications, such as point system, leaderboard, badges, and challenges, etc. Many of these approaches were found effective in promoting motivation (Middelweerd, 2014).

This thesis addresses the problem of player retention in exergames. In particular, and considering the growing popularity of wearable technologies, we focus on exergames that are designed to use wearable activity trackers. This is important due to the potential advantages of these devices in providing information about user activities, and also the observed difficulties they have with regards to retention. More effective and sustainable approaches to activity tracker-based exergames will allow users to take advantage of this technology while forming longer-term healthy habits. Among different approaches, it is suggested that personalized persuasive strategies were effective in increasing intrinsic 
motivation by encouraging activities that are of interest to each particular user (Orji, 2018).

\subsection{Research Approach}

This research aims to answer the following question: How can we keep exergame users motivated, and engaged with the system for longer periods?

We investigated this general question from different perspectives. Firstly, we were interested to determine whether the idea of using wearables in exergames was feasible and motivating. Secondly, we were interested to see if different feature releasing system could have an impact on users' motivation and retention. Thirdly, we aimed to find out if it was feasible to use player modeling in exergames for generating personalized recommendations, and if so, how to build such a player model. Therefore, our research question can be broken down to seven sub-questions below:

RQ1: Is it feasible to use wearable activity trackers for gamification of exercise?

RQ2: Whether game features and their release system can improve engagement over time or not?

RQ3: Is it feasible to provide users with personalized activity recommendations by using continuous player modeling?

RQ4: Can player modeling and gamification techniques improve user engagement/experience towards fitness activities over time?

RQ5: What parameters should exist in the player model? 
RQ6: Which existing models and approaches are effective to recommend specific activities to different users?

RQ7: How the player model could be used to recommend activity?

Four-phase research was carried out to investigate the research questions above. Phase one targeted RQ1, which aimed at evaluating the feasibility of using wearable activity trackers for gamification of exercise and fitness in a short-term study. An exergame with wearable input was developed in research phase one. It was evaluated with an in-lab user tests of 20 participants; Phase two looked at RQ2, which focused on investigating the motivational effects of using a wearable-based gamified system for promoting and sustaining exercise engagement in the long-term, especially on the impact of different ways of releasing game features to users Research phase two extended the application with more features and conducted a 70-day user study of 36 participants.; Phase three covered RQ3 and RQ4, which investigated if using player modeling for personalization of exergaming is feasible in long-term. In research phase three, a new conversation-based, gamified exercise recommendation system was developed, followed by a 60 -day longterm study with 48 participants.; Phase four targeted RQ5, RQ6 and RQ7. to investigate the ways of improving the player model and the recommendation system. There was no formal user study conducted in phase four. Instead, we invited five of our participants from the previous study (from phase 3 ) to try the new version of the system, run a casual pilot study, and provide feedback. 


\subsection{Contributions}

The primary contributions of the work done within this thesis can be summarized as follows:

1. A new theoretical model that describes the effect of various parameters (such as dynamic game features and personal characteristics on engagement and sustainability of the exergames

2. A new architecture for automated personalized activity recommendation for exergames is proposed.

3. A player model for activity recommendation is developed and evaluated through a long-term study for system architecture and a one-time user study to investigate specific activity choices. The result shows the weaknesses of existing methods and the feasibility of applying player modeling and recommendation system to exergames, and how machine learning could be utilized to optimize the player model and the recommendation system.

4. A novel insight into the effect of game features and their release process on retention is described. The result of a long-term user study shows how different ways of releasing game features to users can impact engagement and retention.

5. The feasibility analysis of using commercial activity trackers for exergames is performed. The results show the feasibility and positive motivational effects of using wearable devices in exergames,

6. Two exergames were developed during this research.

7. Through the development and evaluation of the two applications, a number of design recommendations were established. The work within this thesis extends 
the literature on designing exergames (especially on personalized exergames) by further validating previously outlined recommendations as well as adding new recommendations.

8. A further contribution of this thesis is the concise literature reviews of health and fitness and exergame literature presented within Chapter Two, which serve to highlight not only the potential of personalized exergames for promoting physical activity but also the gaps in the literature which this thesis aims to close.

9. Four published and a submitted paper were formed during this research, as described in section 1.5 below.

The research presented in this thesis has been the subject of the following papers:

- (Zhao, 2019): Zhao, Z., Arya, A., Orji, R., "Design of a Activity Recommender System for Personalzied Fitness," to be submitted to ACM Transaction on Computers for Health.

- (Zhao, 2019): Zhao, Z., Arya, A., Orji, R., Chan, G., "Long-term Study of a Personalized Fitness Recommender System using Gamification and Continuous Player Modeling," under revision for ACM Transaction on CHI.

- $\quad$ (Zhao, 2017): Zhao, Z., Arya, A., Whitehead, A., Chan, G., Etemad, S. A., "Keeping Users Engaged through Feature Updates: A long-term study of using wearable-based exergames," ACM Conference on Human Factors in Computing Systems (CHI), 2017.

- (Zhao, 2016b): Zhao Z., Etemad, S. A., Arya, A., Whitehead, A., "Usability and Motivational Effects of a Gamified Health and Fitness System based on Wearable Devices", Lecture Notes in Computer Science (LNCS), Springer, Vol. 9747, pp. 
333-344, (Proceedings of the 18th International Conference on Human-Computer Interaction International (HCII'16), Toronto, Canada), 2016.

- (Zhao, 2016a): Zhao Z., Etemad, S. A., Arya, A., Whitehead, A., "Motivational Impacts and Sustainability Analysis of a Wearable-based Gamified Exercise and Fitness System", Proceedings of ACM CHI Play, EA, pp. 359-365, 2016.

- (Zhao, 2015): Zhao Z., Etemad, S. A., Arya, A., "Gamification of Exercise and Fitness using Wearable Activity Trackers", Advances in Intelligent Systems and Computing, vol. 392, pp.233-240, 2015 [also in: 10th International Symposium on Computer Science in Sport (ISCSS 2015)].

\subsection{Document Layout}

This thesis is organized as follows. First, Chapter 2 introduces the background and state of the art of related areas and reviews of relevant research studies. Chapter 3 presents the proposed solution and the theoretical model of this research, followed by summarizes the overall methodology of the 4-phase research. Chapter 4 describes the study phases 1 and 2 , in which motivational effects of using a wearable-based exergame system (both shortterm and long-term, respectively) for promoting and sustaining exercise engagement were investigated. Chapter 5 introduces the study phase 3 and phase 4 which aims to look at the long-term effectiveness of a personalized gamified fitness assistant system using player modeling, as well as using machine learning for optimizing the player model and generating activity recommendation. Conclusions are drawn in Chapter 6 to summarize the findings of this research. 


\section{Chapter 2: Related Work}

\subsection{Sedentary Lifestyle}

Sedentary lifestyle refers to the type of lifestyle where people engage in very little physical activity or exercise. Typical sedentary activities include sitting, lying down, watching TV, playing video games, and using the computer. The review of sedentary lifestyle studies (Biddle, 2017) shows that sedentary time is linked with an increased risk of diabetes, cardiovascular disease, and all-cause mortality. Specifically, higher levels of sedentary behavior are associated with a $112 \%$ increase in the Relative Risk (RR) of diabetes, $147 \%$ increase in cardiovascular disease, $90 \%$ increase in cardiovascular mortality, and 49\% increase in all-cause mortality (Wilmot, 2012).

According to the Canadian Physical Activity Guidelines (CPAG, 2018), adults should accumulate at least 150 minutes of moderate- to vigorous-intensity aerobic physical activity throughout the week, in bouts of 10 minutes or more. However, the Health Status of Canadians (HSC, 2016) shows the majority of Canadians do not meet recommended levels of physical activity. Just over 2 in 10 adults met the Canadian Physical Activity Guidelines. The low participation rate in physical activities has many causes. Toscos (2011) indicated some potential causes including lack of time, lack of motivation, injury, and physical exertion.

Among different possible solutions, exergames (or gamified exercises) have been popularized as a motivational tool to encourage sedentary or inactive individuals to become more physically active (Biddiss, 2010). 


\subsection{Exergames and Gamified Physical Activities}

Gamification, defined as utilizing game thinking and game mechanics in a non-game context (Deterding, 2011), has been applied to a variety of fields (education, marketing, etc.) to motivate participation and engagement. Especially, it has emerged over the past years as a tool to promote health and wellness (Lister, 2014) (Johnson, 2016) (Edwards, 2016). It helps users to achieve certain fitness goals and increase engagement by adding game features to physical activity.

The effects of gamification have been widely studied by researchers. It has been shown that common game elements such as badges, rewards, leader boards, and avatars are commonly and successfully used to motivate players (Hamari, 2017) (Johnson, 2016) (Mekler, 2017) (Koivisto, 2019). On the other hand, researchers have argued for various areas of improvement in current gamification research and applications, including diversity in themes and context, study duration, and sample size (Koivisto, 2019) (Nacke, 2017) increasing motivation by relying on more intrinsic factors (Nicholson-2012) (Mekler, 2017) and personalization (Nacke, 2017).

Nicholson $(2012 ; 2015)$ discusses the idea of meaningful gamification (or playification) which focuses on playfulness and activities that make sense to each player and rely on intrinsic motivations as opposed to following specific rules to win. Altarriba (2019) build on this idea and propose Situational Play Design (SPD) which is a framework for designing context-based and personalized games.

Studies have shown that gamification in the area of health and fitness could motivate users to become more active (Mueller, 2011). The advantage of gamified exercise over 
traditional forms of exercise is that it is not just a physical activity, but also a form of entertainment. For example, exergames such as Just Dance (and other Wii or Kinect based exergames) offer participants a much higher level of engagement compared to regular exercise, which could reduce the level of perceived exertion. As a result, the level of motivation to stick with the game is also much higher than with traditional exercise (Aloha, 2013). Exergames can not only make exercise seem more enjoyable (Yim, 2007) but can also help improve the overall well-being of older adults (Jung, 2009).

The smartphone game Zombies Run (https://zombiesrungame.com) was a successful example of gamified exercise where headphones were used to interact with users during running. This approach proved to have a positive and motivational effect (Positivegaming, 2016) in promoting people to be more active.

There is also other research focusing on promoting exergame motivation with different approaches. For example, Gobel (2010) proposed a set of personalized exergames that combine the gameplay, and results show that it enhances users' motivation toward the gameplay. Campbell (2008) discussed the concept of everyday fitness games and suggested that for applications that people frequently use in their everyday lives, designs need to be fun and sustainable, as well as to adapt to behavioral changes. Wylie (2010) indicated that with the use of smart-phones, gamified components such as a leaderboard, achievements, and challenge amongst friends are effective ways of motivating and encouraging users to reach their personal goals and track their physical fitness activities. Hamari (2013) investigated how social factors affect users' intention to continue using the gamified system, and their results also indicated that social factors are strong predictors for game motivation. Schwenk (2014) implemented a Virtual Reality (VR) 
game-based system prototype for balance rehabilitation, in which wearable sensors and interactive user interfaces were involved for real-time visual feedback. Findings support that real-time feedback is an important factor that could affect the user's in-game experience.

The approaches above showed the potential of using different technologies, such as wearables, VR, as well as personalization, in promoting exergame motivation. However, none of them considered the long-term effectiveness of those proposed systems.

On the other hand, (Smeddinck, 2015) conducted a five-week study comparing exergames with traditional therapy. Results showed that a significant increase in the measure of functional reach when training with exergames. Macvean (2013) reported a seven-week study on user's physical activity, motivation and behavioral patterns on using exergames, and suggested that longitudinal studies are necessary for evaluating motivational effects since it ensures that the intensity of a user's behavior is appropriate and sustained. These two studies aimed to look at long-term exergame motivation, but the game used in their studies was relatively simple and did not show the effectiveness of different technologies in designing exergame.

\subsection{Wearable Devices for Health and Fitness}

In recent years, rapid advances have occurred in relatively low-cost wearable devices that help users to monitor their physical activity and become more active (Piwek, 2016). Devices such as Fitbit, Apple Watch, and Android Wear watches can measure a variety of activity-related outcomes including steps, distance, heart rate, active minutes, calories, and sleep. Additionally, users can access the app and web interface to socialize with 
friends and complete group challenges. Many devices have shown high validity and reliability (Evenson, 2015) and a growing amount of research has successfully incorporated different devices into technology-oriented lifestyle interventions to increase physical activity (Coughlin, 2016). Users can track minutes of physical activity, steps per day, and floors climbed per day, enabling them to receive feedback on their activity.

Research has indicated that it is feasible to use consumer wearable devices to promote physical activity. For instance, Washington (2014) conducted a study of a Fitbit-based physical activity intervention among 11 healthy college students, and found participants increased overall step counts $23 \%$ overall. Wang (2015) conducted a randomized controlled trial of a 6-week Fitbit-based physical activity intervention. A significant within-group increase of 4.3 minutes per week of moderate to vigorous-intensity physical activity was observed in the comparison group (with Fitbit).

Ashe (2015) conducted a randomized controlled trial of a 6-month Fitbit-based physical activity intervention. There was an average between-group differences in a weight loss of $4.3 \mathrm{~kg}$ in favor of the intervention. Chung (2016) conducted a randomized controlled trial comparing the use of Fitbit and smartphone app vs. Fitbit alone to promote physical activity. One-day challenges were found successful in increasing daily steps.

The research above indicated the potential of using wearables in physical activity intervention to promote healthier daily life. However, most of the studies used default applications of those activity trackers, very few research has considered using those trackers in developing exergames with more game features. 
Moreover, in recent years, the use of wearable sensors in HAR (Human Activity

Recognition) is becoming popular (Lara, 2013), in which most of the measured attributes are related to the user's movement (e.g., using accelerometers or GPS), environmental variables (e.g., temperature and humidity), or physiological signals (e.g., heart rate or electrocardiogram). These data are naturally indexed over the time dimension, consistent, and convenient to access, which could be utilized in modeling and predicting a user's daily activity patterns.

\subsection{Player Models}

\subsubsection{Personality Models}

Personality traits typically are understood to be stable behavior patterns present since young adulthood (Wiggins, 1992). Developing an understanding of personality typology is a useful way to improve the knowledge of motivation and behaviour of self and others (Judge, 2002). There are many different personality models and theories, and each one offers a different perspective. For example, the Big Five personality characteristics (John, 1991) represent the most widely used model for generally describing how a person engages with the world.

The Myers-Briggs Type Indicator (MBTI) is one of the most popular psychological assessments, which is based on 16 personality types. The MBTI test is widely used in business settings and retreats for team building, as well as in communication and educational (Coe, 1992). Moreover, studies showed that people's personality type and their gaming preferences were highly linked (Tondello, 2016). 


\subsubsection{Gamer Models}

To personalize games, gamer type models are frequently discussed and applied paradigm in the gaming community (Ferro, 2013). Gamer types characterize gamers according to their preferences for general game mechanics. These models suggest that certain gamer types are receptive to specific game mechanics. Therefore, games that contain game mechanics that fit the players' type are hypothesized to lead to a better player experience.

The general concept of gamer type models shares similarities with personality trait models, such as the Big Five model (Mccrae, 1992): Both aim at capturing stable individual differences, which can be used for describing humans and explaining differences in behavior and underlying attitudes. In that sense, personality trait models can be seen as a higher-level conceptualization of individual differences (not directed at certain areas or behaviors), whereas gamer type models are more specific and focused at explaining differences in player behavior, experience, and emotions. Gamer type models could be used for planning new or adapting existing games to target audiences for improving the player experience and facilitating long-term engagement with the games (Busch, 2016).

One of the oldest and most frequently used player type models is the one by Bartle (1996), which identified four player types, analyzing players of Multi-User Dungeons (MUDs) regarding what they wanted out of a MUD. Despite its high prevalence, Bartle's player type model has some shortcomings: He proposed that each player has some overall preference for one of the types (mutually exclusive) and did not empirically test the model on the independence of the types or on psychometric quality criteria. 
Yee (2006) used a factor analytic approach of questions based on Bartle's player types. His analysis identified three main components (with ten subcomponents) of player motivation with weak correlations. Like Bartle's model, Yee's components have a limited focus on only MMORPGs. Therefore it is probably not suitable for a broad range of game genres. Furthermore, the predictive validity of this model has not been investigated.

A wider perspective regarding game types is included in the first Demographic Game Design model (DGD1) (Bateman, 2005), which is an adoption of the Myers-Briggs type indicator (MBTI) (Myers, 1977) to games. The four-player styles Conqueror, Manager, Wanderer, and Participant are proposed in the DGD1. This model is qualitatively supported by a series of case studies. Besides player styles, the studies also investigate hardcore and casual players, which are present in all four-player styles. In the second Demographic Game Design model (DGD2) (Bateman, 2011) the hardcore/casual dimension, as well as different skill sets and the preference for single and multiplayer is explored in more detail.

Emerging from an empirical evaluation of a health game for younger adults, (Xu, 2012) developed five player types which include both, motivational and behavioral factors. A user type model related to gamified systems was proposed by Marczewski (2015). He proposes Disruptors, Free Spirits, Achievers, Players, Socializers, and Philanthropists. These types differ in the degree to which they can be motivated by either intrinsic (e.g., self-realization) or extrinsic (e.g., rewards) motivational factors. Additional work that used player modeling is Drachen (2009), where tools were used to extract gameplay information (implicit implementation) from the game Tomb Raider: Underworld and 
processed these data with neural networks to obtain playing styles (Pacifist, Runner, Veteran, and Solver). During this process, the authors extracted some information to make this classification, like the cause of death, completion time, and the number of deaths. This information is applicable to the optimization of game design features.

Another example that studied game design improvement based on adaptive analysis is (Pedersen, 2010). They looked for correlations between player's satisfaction and level characteristics of the game Infinite Mario Bros, such as the presence of gaps, blocks, and enemies. This allowed the authors to maximize player satisfaction.

The BrainHex model (Nacke, 2011) is a relatively new model but has been validated with large numbers of participants. It is a promising approach, supplementing existing research with a more diverse array of player types and it has been used in several recent studies. In BrainHex, player types were not mutually exclusive. The corresponding BrainHex questionnaire assesses the players' dominant player type (primary type) and subtypes. In other words, instead of putting each player into a single gamer category, the BrainHex model provides each user with detailed scores of each individual gamer type, which allowed us to adjust the weight of different game elements according to scores, rather than simply using one type. It also connected player types to the game elements.

Moreover, the Hexad model (Tondello, 2016), which is of particular interest in our work, is a gamification player types model created for mapping user personality onto gamified design elements. We considered using Hexad in our player model because it targeted gamified systems in particular. It proposes six player types, and the player types of individuals are correlated with their preferences for different game design elements. 
Design guidelines for tailoring persuasive gameful systems to each gamification player type have also been studied (Orji, 2018).

\subsubsection{Exerciser Models}

Similar to gamer types, personality type also plays an important role in determining people's fitness tastes. Some people may prefer swimming laps solo while others enjoy attending a rowdy group-cycling class. These preferences have less to do with people's physical characteristics and are affected more by personalities. Matching activities to personality type has been shown to have real-world relevance (Courneya, 1998).

Research suggests that people who engage in personality-appropriate activities will stick with the activities longer, enjoy their workout more and have a better overall fitness experience (Rhodes, 2006).

Brue (Brue, 2008) created a system, which is a personality centered approach to exercise grounded in the personality type framework popularized by the Myers-Briggs Type Indicator (MBTI) instrument. She took the MBTIs - Introversion (I) or Extraversion (E), Intuition $(\mathrm{N})$ or Sensing $(\mathrm{S})$, Thinking $(\mathrm{T})$ or Feeling $(\mathrm{F})$, and Judging $(\mathrm{J})$ or Perceiving $(\mathrm{P})$ - and reworked them into an easily maneuverable color-coded fitness personality model, which aims to help people discover the best approach to exercise based on personality type. Brue indicated that knowing more about the personality and people's likes and dislikes can make it easier to plan and, more important, be satisfied with exercise, which makes it more likely people will continue to engage in it over the long term.

The 8-Colors is based on 8 preference pairs each corresponding to a Color. By understanding Fitness Personality, people gain an understanding of their motivational 
patterns, preferred interactions, and environments, and can more effectively choose specific forms of physical activity that are best for them and they will stick with. For instance, some people are traditional and conservative in their approach to exercise while others seek variety and cutting-edge information. Some enjoy solitude and consider exercise a moving meditation while others prefer a fast-paced class with energetic music.

In the 8-color system, eight types of fitness colors were introduced in which reds are quick responders in the physical world. Whites like to plan and are visionary types who like calm spaces and don't like to be rushed. Greens are nature lovers who like to be outdoors. Golds are traditional, conservative types. Saffrons value individual expression. Blues are safety oriented and are good at creating their own space in a gym. Purples are routine-oriented. Silvers like exercise to be disguised as fun, or at least a fun way to meet others. Overall, people with different fitness color were motivated by different factors, as well as were linked to some suggested activities.

Therefore, the 8 Colors of Fitness model was initially used in our proposed system for suggesting different types of activities. This model is one of the few that use personality type as the basis of activity recommendation and is suggested by other researchers and practitioners (Taylor, 2017) (Bayne, 2012). The detailed model description is outlined in the Appendix.

However, because the 8-color-of-fitness system was used initially as a model to suggest activity types, the recommendations of activity types that our system generated were mostly based on personalities. This was due to the fact that the research group did not find any other alternatives and needed to rely on a fairly acceptable method. Therefore, 
the method of mapping player model to the right type of activities for recommendation can be further investigated.

\subsection{Recommendation System for Physical Activities}

As for building recommendation systems, various methods exist. Bruke (2002)

distinguishes four different categories of recommendation techniques based on knowledge source:

- Collaborative: The system generates recommendations using only information about rating profiles for different users.

- Content-based: The system generates recommendations from two sources: the features associated with products and the ratings that a user has given them.

- Demographic: A demographic recommender provides recommendations based on a demographic profile of the user.

- Knowledge-based: A knowledge-based recommender suggests products based on inferences about a user's needs and preferences.

Hybrid recommender systems, another type, are basically a combination of multiple recommendation systems to overcome some of the shortcomings of each.

When designing a recommender system for exercise and fitness activities, there were some practical constraints which make it difficult to directly use any of the wellestablished recommender system algorithms. For instance, most types of recommendation systems work based on ratings on items. In the field of physical activity, 
no ratings exist. This is due to the feeling of individuals concerning a specific exercise activity is hard to measure.

Therefore, in our search of finding closely related research, we come across very limited literature that discusses the use of recommender systems in sports and exercises. However, the idea of Personalization in physical activity has been studied by many researchers. For example, Guo (2017) proposed a system that recognizes different types of exercises and interprets fitness data (motion strength and speed, etc.) to an easy-tounderstand exercise review score, which aims to provide a workout performance evaluation and recommendation. Though it achieved $90 \%$ accuracy for workout analysis, it focuses only on recognizing fitness activities and not personalizing or gamifying them.

He (2014) introduced a system designed to be context-aware for physical activity recommendation. It focuses on selecting suitable exercises for individualized recommendations. A smartphone application was developed that could generate individualized physical activity recommendations based on their database of physical activity. The focus of their work is to recommend different types of activities but does not take into account personal details such as proper time, location, and intensity, or any gamified elements.

Broekhuizen (2010) proposed a framework called PRO-fit, which is another example that employs machine learning and recommendation algorithms to track and identify user's activity by collecting accelerometer data, synchronizes with the user's calendar, and recommends personalized workout sessions based on the user's and similar users' past activities, their preferences, as well as their physical state and availability. They 
highlighted that many applications nowadays are more focused on tracking user's activities, but do not provide a recommendation system that would help users choose from activities based on their interests and accomplishment of goals. Therefore, they were motivated to design the personalized fitness assistant framework that acts as a motivator and organizer for fitness activities, making it easier for users to create and follow their workout plan and schedule the sessions according to their availability and preference. However, their system was based on prefixed recommendations which do not involve a player model.

Rabbi (2015) introduced a smartphone application "MyBehavior" that generates personalized health feedback from physical activity and food log data. A 14-week study showed improvement in physical activity and a decreases in food calorie consumption when using the application compared to a control condition. This work was a novel approach that provides personalized suggestions by learning the user's behaviors, which was close to our proposed idea. However, MyBehavior was not an exergame. It did not look at the impact of gamification elements or any feature release method. Their approach in generating recommendation was different from player modeling.

\subsection{Summary and Gap Analysis}

Overall, existing research shows the feasibility of using gamification technology for promoting physical activities. Off-the-shelf wearable activity trackers have considerable potential to be utilized in the gamification of exercises. Player modeling techniques and personalized activity recommendations have drawn significant research attention. 
However, existing work on gamified and persuasive games in health and wellness are mostly limited due to the one-size-fits-all approach, in which the same fixed content is generated for all target users. Initial attempts at personalization are mostly limited to a small set of static parameters about the user (simple demographic information such as age, gender, and occupation, determined before the study and does not change) which makes their "recommendations" static, less effective and personalized.

Moreover, as we can see from existing work, the effectiveness at promoting the desired behavior was mostly evaluated based on a single point of use and feedback (short-term). However, when it comes to exergaming or other physical activity-related interventions, it is important to consider the long-term effectiveness and sustainability of the system since it takes time to form a habit of exercising.

Finally, due to those practical constraints which make it difficult to design a recommender system for exercise and fitness activities, very limited research has been conducted in this area.

To fill these theoretical and technical gaps, our research aims to answer the following major question:

How can we keep exergame users motivated, as well as engaged with the system for longer periods?

This question can be further broken down into a series of more specific questions:

RQ1: Is it feasible to use wearable activity trackers for gamification of exercise? 
RQ2: Whether game features and their release system can improve engagement overtime or not?

RQ3: Is it feasible to provide users with personalized activity recommendations by using continuous player modeling?

RQ4: Can player modeling and gamification techniques improve user engagement/experience towards fitness activities over time?

RQ5: What parameters should exist in the player model?

RQ6: Which existing models and approaches are effective to recommend specific activities to different users?

RQ7: How the player model could be used to recommend activity?

To answer these questions, we designed and developed a system based on a comprehensive player model for personalization, recommending 24/7 personalized activities, using gamified elements and wearable devices. To the best of our knowledge, none of the existing studies has conducted a long-term user study to evaluate their systems. 


\section{Chapter 3: Proposed Theoretical Model, Solution, and Research}

\subsection{Theoretical Framework}

\subsubsection{Related Theories}

As we discussed in the previous chapter, our research brings together multiple areas of study, including exergames, wearable technologies, adaptive and dynamic games, and player modeling.

The basis of this research and our theoretical framework is the notion of exergames and gamification of exercise activities, particularly using wearable technologies. Despite the growing interest in persuasive games and gamified systems for motivating health-related behaviors (Orji, 2018; Orji, 2017), the majority of persuasive games employ a one-sizefits-all approach to their design (Busch, 2015). To the best of our knowledge, very little research has been done to evaluate player preferences and motivations for personalizing the experience, specifically in the context of exergames.

Adaptation can be defined as the ability to make appropriate responses to changed or changing circumstances (Charles, 2004). Adaptivity within games can be implemented by dynamic difficulty technologies (Miller, 2004), but there are several other ways in which adaptivity can work. For example, in helping players avoid getting stuck, adapting the gameplay to the player's preference, or generating personalized game content to target individual users.

Dynamic game difficulty balancing (DGDB) (Hunicke, 2005), as the most widely used example, is the process of automatically changing parameters, scenarios, and behaviors in 
a video game in real-time, based on the player's ability, in order to avoid making the player bored (if the game is too easy) or frustrated (if it is too hard).

There are many practical examples of the use of adaptive technologies in current commercial games and game research (Vaughan, 2016), which demonstrates the considerable potential of this technology - particularly research on Intelligent Interfaces (Sullivan, 1994) and Intelligent Tutoring Systems (Anderson, 1985), which were very focused on user adaptation. However, the number of studies focusing on using adaptive technologies in exergaming is limited.

Player modeling is the study of computational models of players in games (Yannakakis, 2013). This includes the detection, modeling, prediction, and expression of human player characteristics which are manifested through cognitive, affective, and behavioral patterns. Different from player profiling, player modeling is aimed at modeling dynamic phenomena during gameplay, while player profiling focused on the categorization of players based on just static information.

Furthermore, player modeling is built on dynamic information obtained during gameplayer interaction, but they could also rely on state player profiling information. (personality, cultural background, gender, age, etc.) (Begis, 2000).

The primary goal of creating player models is for the game to recognize and adjust its content (gameplay, difficulty, storyline, etc.) to target each individual player. A player model contains a set of attributes relevant to the personalization, which in general includes a player's profile, game preferences, and personality data. The physical characteristics of the player are essential for exergames personalization. 


\subsubsection{Proposed Theoretical Model}

While the existing studies have addressed many aspects of these diverse fields, as we discussed earlier, they have not properly integrated to develop engaging and sustainable exergames. For example, the effect of various game features and adapting the game to player needs and interests have not been investigated in the context of exergames.

In this chapter, we describe our proposed system architecture and the related research method. This proposal is based on our new theoretical model developed after reviewing the related work, consisting of the following principles:

- Advances in wearable technologies allow game designers to use commercially available activity tracking sensors and mobile devices as a major element of exergames.

- A game with a static design, no matter how interesting, will lose its attraction after a while. As such, it is important to "add new features" over time to keep players engaged.

- While different methods exist for adding dynamic features to games, designers have limited ability to provide new features constantly, and there is no guarantee that they will be attractive to users. An alternative (or complementary) approach is to dynamically modify the game by "adapting to the player."

Our theoretical model is demonstrated in Figure 3.1 below. 


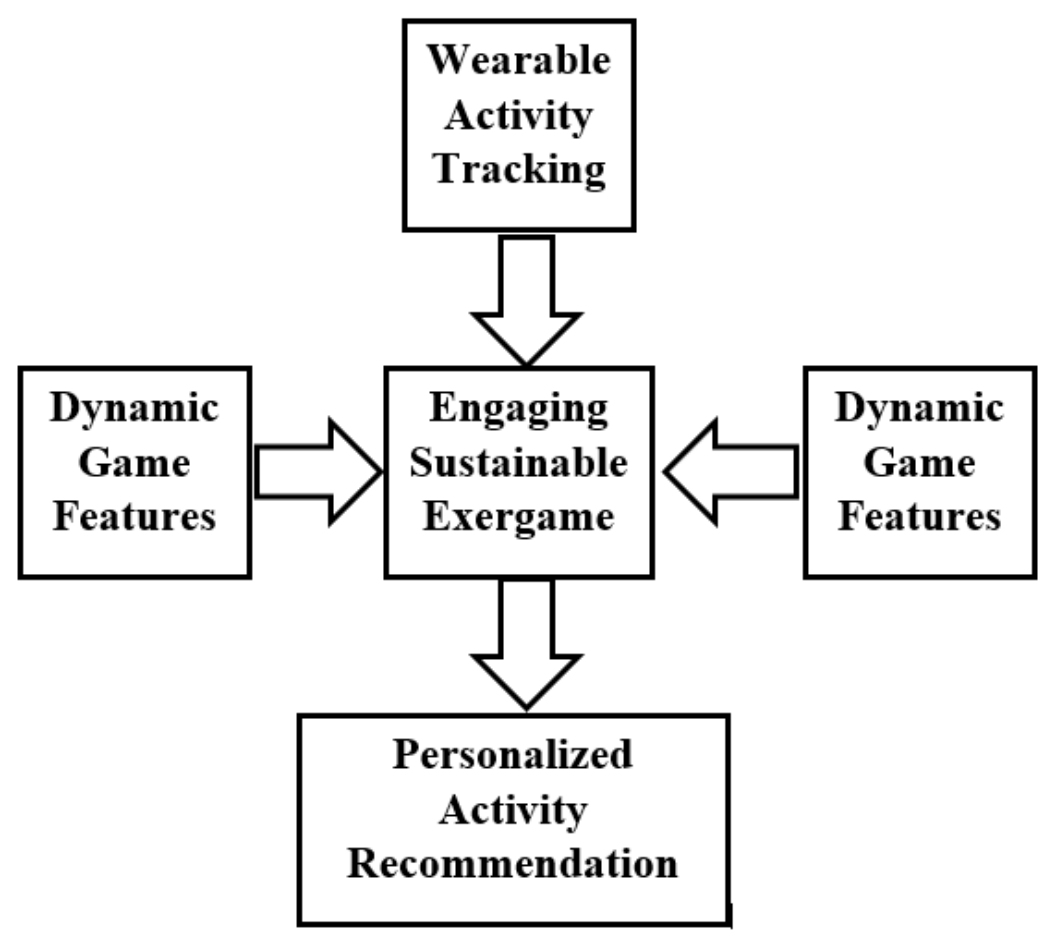

Figure 3.1: Theoretical Model

\subsection{Proposed System Architecture}

As mentioned in previous sections, bringing wearable activity trackers or smartwatches into exergames, as well as the personalization of exergames, is being proposed as a solution to the research problem. Therefore, a wearable-based exergame with a comprehensive player model for personalization, recommending customized activities, is proposed as a potential system for further investigation. Figure 3.2 below shows the overall architecture of the proposed system.

The proposed system contains mainly three components: a player model, a recommendation engine, and a game generator. The player model takes different types of user data and predicts user preference for physical activities, and finds proper time and 
location for recommending activity sessions. It consists of several sub-models that covered the user's general, personality, and daily activity data. The recommendation engine utilized the output of the player model and generates customized Physical Activity (PA) session recommendations for individual users (which includes the proper time, location, intensity, and the potential type of physical activity). The game generator adds customized game elements to the recommendation and generates the final game content that users can interact with.

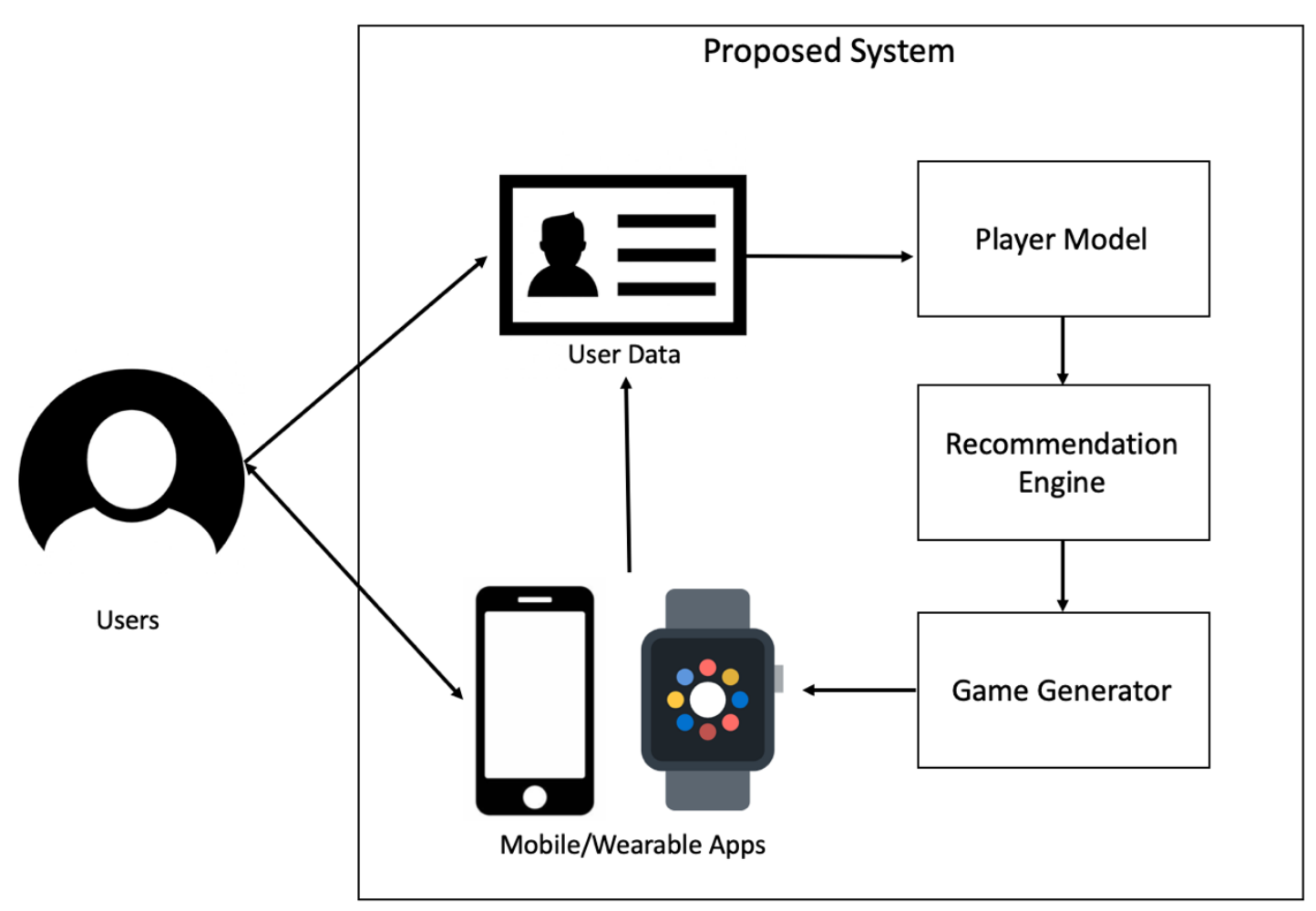

Figure 3.2: Proposed System Architecture

Wearable activity trackers or smartwatches are used in the system for tracking user's activity, and for introducing diverse interactions. The combined use of the mobile and the 
wearable applications will allow users to interact with the system with different modes. The detailed design and development of the system are introduced in Chapter 5.

Overall, a wearable-based exergame system, with a comprehensive player model for physical activity recommendation, and game customization is proposed as a solution to the exergame retention problem.

\subsection{Research Approach}

\subsubsection{Research Questions and Hypotheses}

As described above, the overall focus of the research is to look at exergame user motivation and long-term engagement. Based on the proposed system described in the previous section, the primary research question could be investigated from several perspectives. Such as, how wearable activity trackers could be used in exergames and how it could affect the user's motivation and engagement (RQ1); How game features and their release system could have an impact on long-term engagement (RQ2); Whether it is feasible to provide users with personalized activity recommendations by using player modeling (RQ3); If so, can this personalization actually improve long-term engagement or not (RQ4); Afterwards, if the idea of player modeling is feasible, what features should exist in the player model to make it better? (RQ5); and how we can compare our model to existing works? (RQ6); Finally, how the player model could be used to recommend activity? (RQ7).

Figure 3.3 below summarizes the research questions we aimed to investigate. Our overall hypothesis can be summarized as: 
Adding wearable activity trackers, gradually releasing game features, as well as personalization of exergames could increase user motivation, as well as have a positive impact on user's long-term engagement in exergame system.

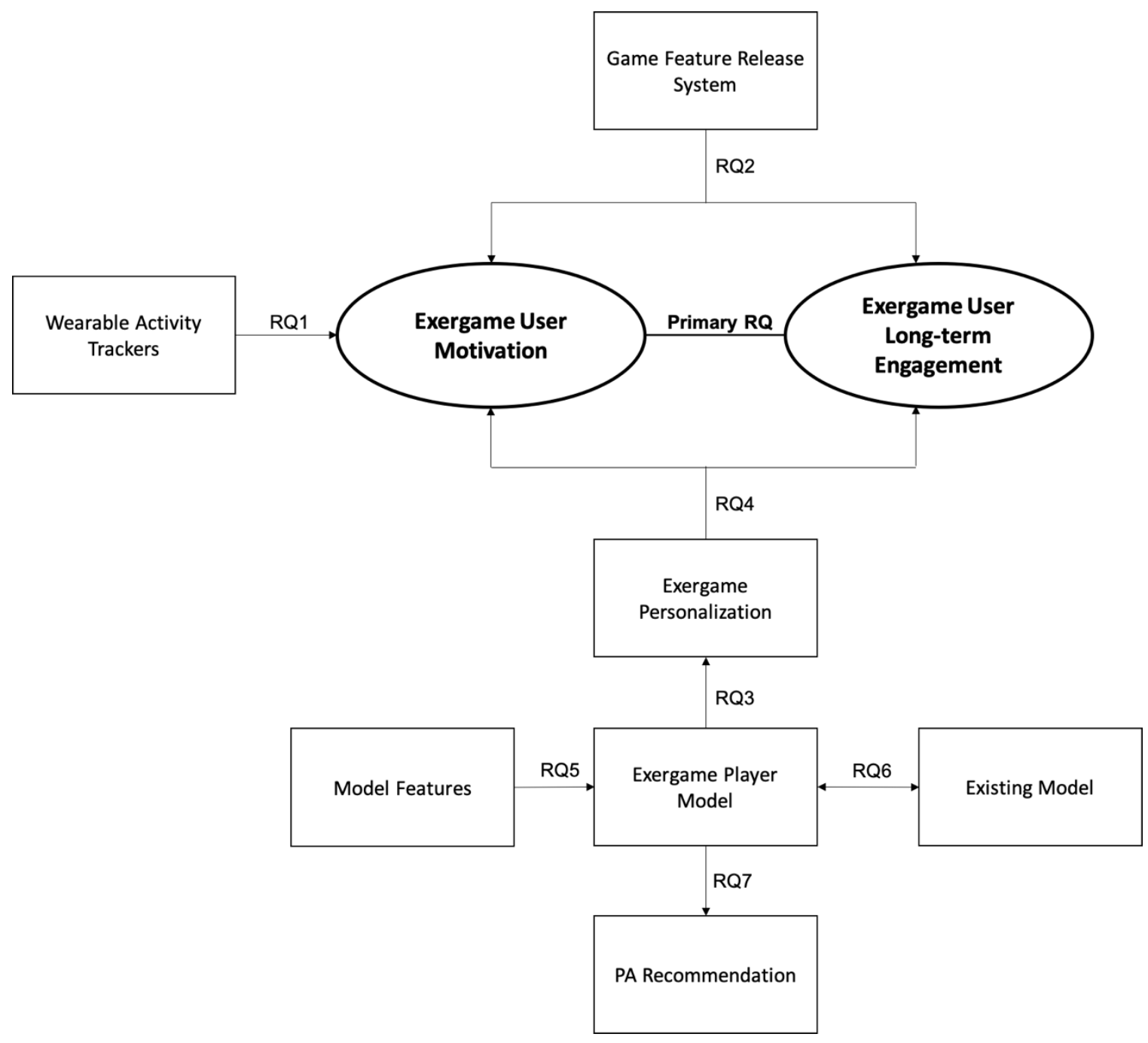

Figure 3.3 Proposed Research Questions

Therefore, the independent variables we aimed to look at in this research including wearable activity trackers, game feature release system, and personalization. Among them, wearables and game feature release systems are relatively uncomplicated variables 
that can be evaluated with direct user research. Personalization, on the other hand, is a more general idea and required more detailed design and investigation. To be specific, the personalization technique we considered in this research is player modeling. Therefore, all the research questions related to personalization were built around the player model, which including model feature selections, compared to the existing model, and how to use the model for PA recommendation.

In this research, we chose to design and develop our own exergame system instead of using existing commercial games. This was due to the fact that existing games can introduce multiple variables such as aesthetics and gameplay features that are out of our control but can affect the results. The game features and the UI of the experiment system were relatively simple so we could focus on variables that we were investigating, i.e. personality model, feature change methods, and gamification.

\subsubsection{The Four Phases Research}

As discussed in previous sections, wearable activity trackers, and the game feature release system are relatively uncomplicated independent variables that can be evaluated with direct user research. Conversely, personalization is a more general idea and in this research, we focused on using a comprehensive player model to personalize the user's exergame experience. Therefore, the proposed research approach is divided into four phases of studies to gradually design, develop, and optimize the system, as well as to investigate the research questions proposed above, as summarized in the table below: 
Table 3.1: Mapping of Research Questions to Research Phases

\begin{tabular}{|c|c|}
\hline Research Phase & Research Questions \\
\hline 1 & RQ1 \\
\hline 2 & RQ2 \\
\hline 3 & RQ3, RQ4 \\
\hline 4 & RQ5, RQ6, RQ7 \\
\hline
\end{tabular}

Phase 1: To evaluate the feasibility of using wearable activity trackers for gamification of exercise and fitness in a short-term study (RQ1).

Phase 2: To investigate the motivational effects of using a wearable-based gamified system for promoting and sustaining exercise engagement in the long-term. Especially to look at the impact of different ways of releasing game features to users. (RQ2).

Phase 3: To investigate if using player modeling for personalization of exergames is feasible in the long-term (RQ3 and RQ4).

Phase 4: If the result of Phase 3 is positive, to investigate the means of improving the player model and the recommendation system (RQ5, RQ6, and RQ7).

To be specific, for research phase 1 , in order to investigate the feasibility of using wearable activity trackers in exergames, a smartphone game application is designed and developed which uses wearable devices as an input system. The game supports multiple wearable devices that are commercially available to end-users, and multiple types of activities were included in the gamified experience. These activities are performed as inputs to the mobile game. User studies were conducted to evaluate the effectiveness of the combined use of games and wearable devices in promoting exercise. The usability of 
the proposed approach and the effects of different factors within the system were also evaluated.

In research phase 2 , the design and findings of a study on the motivational effects of using wearables-based games to promote daily exercise in the long-term are presented. Furthermore, we have investigated user behaviors, usage patterns, engagement, and parameters that affect them. An updated version of exergame (based on Phase 1) was developed with an accompanying wearable device, for which different variations of application updates were pushed out periodically over a 70-day period.

Phase 3 introduced a gamified 24/7 personal fitness assistant system which 1) tracks, recognizes and predicts user daily activity patterns dynamically, 2) provides personalized recommendations on certain types of exercise (taking into consideration the proper time, location, the type of exercise and intensity, etc.,) and 3) generates personalized gamified experience for an individual user. A 60-day long-term study was conducted to investigate the feasibility of the proposed system, and the long-term effects. Specifically, the results show the feasibility of generating personalized exercise recommendations using player modeling.

Based on the positive results from research phase 3 , research phase 4 focused on the optimization of the proposed system. Firstly, a survey is conducted to gather data regarding people's exercise preferences. Then based on the survey results, we conducted a feature importance experiment to determine what parameters were more important in predicting user preferences for physical activities. Afterward, we re-designed and implemented the recommendation system based on the prediction model and added a 
self-update feature to the system to make it dynamically adjustable. We compared the proposed model to an existing model on the accuracy in predicting user's PA type preference

\subsubsection{User Study}

In order to gradually investigate the research questions, four user studies were carried out within the four phases of research:

- For research phase 1, in-lab user tests of 20 participants were conducted to evaluate the effectiveness of the combined use of games and wearable devices in promoting exercise, and to investigate the usability of the proposed approach and the effects of different factors within the system.

- In research phase 2, a 70-day user study of 36 participants was designed to verify the hypothesis that adding different game features and gradually releasing them can positively affect user engagement and retention.

- In research phase 3, a 60-day long-term study with 48 participants was conducted to show the feasibility and effectiveness of using player modeling technique in the personalization of exergame.

- Due to time constraints, there was no formal user study conducted in phase 4 . Instead, we invited five of our participants from the previous study (from phase 3) to try the new version of the system, and participate in a casual pilot study and provide feedback. 


\subsubsection{Data Collection}

In general, the experiment data for this research is obtained from either the questionnaire/surveys or from in-app behavior tracking.

Typically, we asked participants to complete a pre-study questionnaire before the in-lab session (short-term) or before providing them with the application and devices (longterm), and a post-study questionnaire at the end of the sessions (short-term) or when returning the devices (long-term). For research phase 4, the survey was conducted online because no associated user study was performed.

For in-game data collection, we used Google Analytics API to track participants' comprehensive in-app behavior data, including screen views and tapped events with an associated timestamp. We used iOS HealthKit API or Google Fit API (depend on the application platforms) to track user daily activity data. There were also some pop-up questions when immediate feedback after an activity session was required, etc.

To ensure consistency, all the questions we asked in the user studies were based on 7point Likert scales (from $1=$ strongly disagree to 7 = strongly agree). One-way betweengroups analysis of variances (ANOVA) and post-hoc Tukey-Kramer HSD tests was conducted to analyze the main effects between groups for all studies. The alpha was set at 0.05 for all statistical tests.

Qualitative data were collected from either open-ended questions in the surveys or from casual interviews with participants. 


\section{Chapter 4: Wearable-based Exergame (Research Phases 1 and 2)}

\subsection{Short-term Study}

The first phase of our research included a short-term study for feasibility of using commercial wearble devices in exergames.

\subsubsection{Overview}

Gamification of exercise has become a popular topic due to its motivational and engagement effects, which intends to result in increased exercise, activity, health, and fitness for everyday users. At the same time, wearable technologies have become a fastleader board growing industry, providing consumers the ability to conveniently track their state of health and fitness efforts. Consequently, we propose that off-the-shelf commercial wearable technologies have great potential to be applied in gamification of fitness and exercise. In this study, we utilize this concept through the design and implementation of a smartphone game application that uses wearable devices as input systems. The game supports two different wearable devices that are commercially available to end-users, and three types of activities were included in the gamified experience. These activities are performed as inputs to our mobile game. User tests evaluate the effectiveness of the combined use of games and wearable devices in promoting exercise. The usability of our proposed approach and the effects of different factors within the system are also evaluated. 


\subsubsection{Application Design and Implementation}

The iOS application StrayBird was implemented for this study. The application communicates with wearable activity trackers in real-time and uses data received from these devices as inputs for the game. The game requires that the player engages in running, cycling, or rope skipping motion to control the movements and actions of the principal character, which is a bird that has fallen behind his travelling companions. The objective is to regroup with the flock before time runs out and before the group reaches its intended destination. There are also physical and environmental parameters that may affect the movement behaviour of the bird and flock. The game features a mission-based structure and a point-based system. It is based on a linear progression structure and consists of unlockable levels that increase with slight difficulty and differ to a certain degree in gameplay. For game feature selection in this application, we included some common game elements such as badges, rewards, leader boards, and avatars which were commonly and successfully used to motivate players (Hamari, 2017) (Johnson, 2016) (Mekler, 2017) (Koivisto, 2019). Figure 4.1 presents some screenshots from different sections of the application, namely the choice of exercises, game view, and result view.

We have connected two devices to this application: Apple Watch and TI SensorTag. The former is an example of a high-end off-the-shelf product, while the latter is an example of a simple do-it-yourself system that can be adjusted for different applications. The data from the Apple Watch can be directly transferred to iOS HealthKit via Bluetooth and the app will be able to obtain data from HealthKit, though there are approximately 10 seconds of uncontrollable delay during the data transmission. For the TI SensorTag, we 
could directly access the raw data from its accelerometer, so we implemented an algorithm to calculate the speed from raw acceleration data.
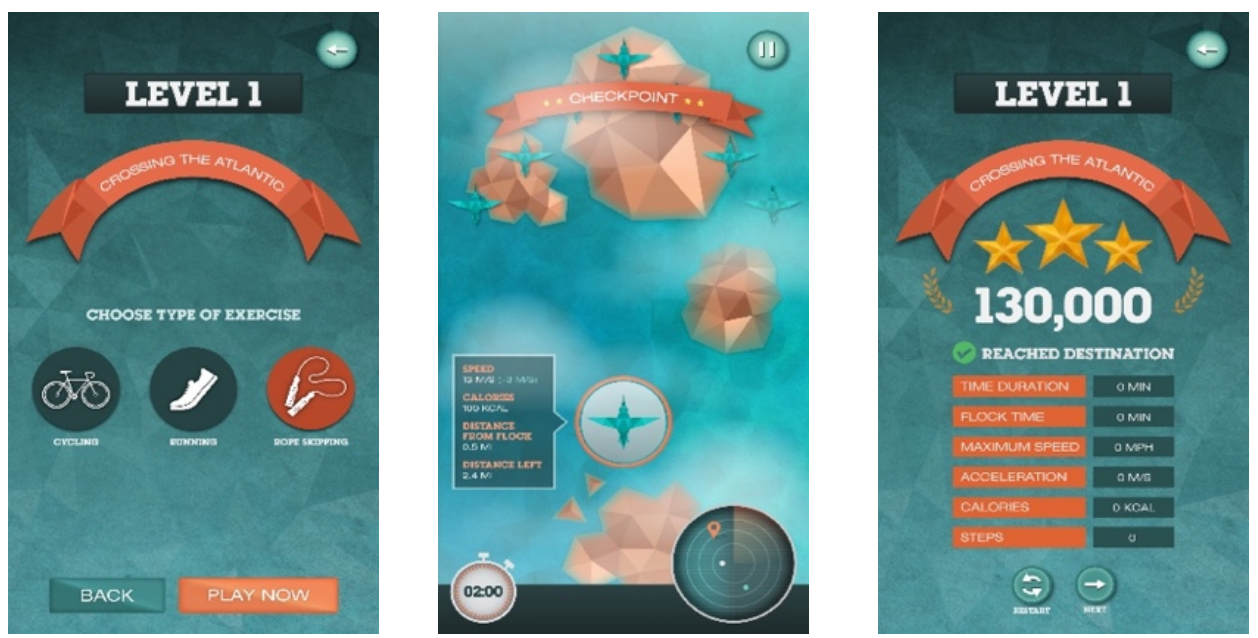

Figure 4.1: Screenshots from Our Application. From left to right: choice of exercises, game view, and result view.

\subsubsection{User Study Design}

20 participants participated in our study, each of whom performed two sessions of exercises. The two wearable products and three different games (running, cycling, and rope-skipping) were randomly combined and assigned to participants. The Apple Watch was not used with cycling because it can only be worn on the wrists. Subjects took part in a maximum of two different trails. Each trial took 10-15 minutes followed by a 10minute rest and recovery period. Each exercise mode prompts the user to perform a specific exercise (run, cycle, or skip ropes). Figure 4.2 shows our three exercise modes. During each game, metrics such as speed, traveled distance, and calorie expenditure are recorded from the user. Once an exercise was completed, during the rest period, participants were asked to fill a questionnaire for each game that they played. This included questions on the level of enjoyment, fatigue, sense of accomplishment, motivation for more exercise, and usefulness of the application. 

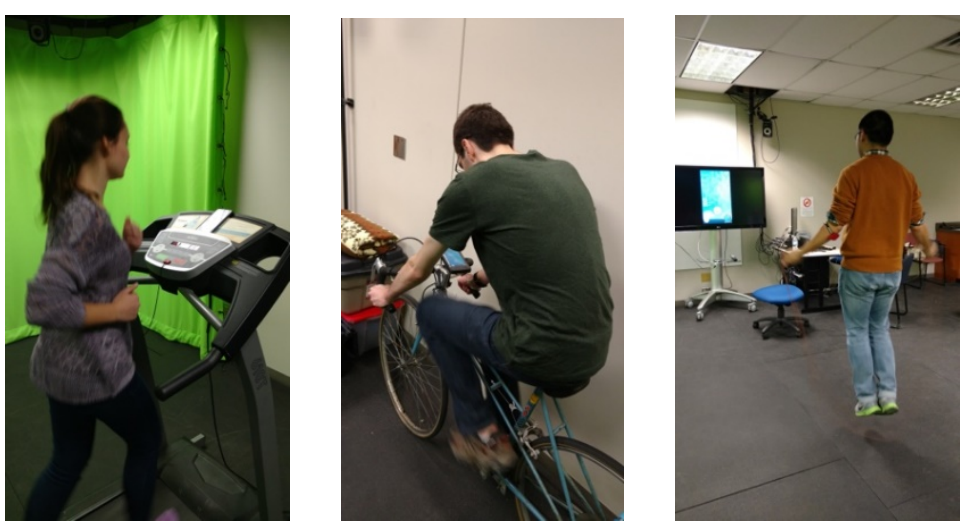

Figure 4.2: Three Types of Exercises in Our User Study: running, cycling, and rope skipping.

\subsubsection{Result and Analysis}

Two-way between-groups analysis of variances (ANOVA) was conducted to analyze the main effects of different game modes (exercise and device) and different game-play parameters (perceived by users). The investigated parameters are enjoyment, fatigue, accomplishment, motivation, usefulness, satisfaction, and preference. A 7-point Likert scale was used in this study. Note that for all the Likert scale values we used in this series of study, the detailed presentation of the questions were listed in the Appendix. We used $\mathrm{R}$ to run the analysis. The different combinations of exercise and wearable devices are presented and labeled for future reference (Table 4.1).

Table 4.1: Combination of Exercises and Wearable Devices

\begin{tabular}{|c||c|c|}
\hline & Apple Watch & TI SensorTag \\
\hline \hline Running & A & B \\
\hline Cycling & - & C \\
\hline Rope Skipping & D & E \\
\hline
\end{tabular}

20 volunteers participated in this study. 10 were males and 10 were females. Their average age was 25.25 with a standard deviation of 4.27 . The average weight was 62.35 
$\mathrm{kg}$ with a standard deviation of 11.9 . The average height was $170.65 \mathrm{~cm}$ with a standard deviation of 6.80. Their average hours of exercise per week was $4.53 \mathrm{hr}$ with a standard deviation of 2.20. Their average hours of playing computer/mobile games per week were 4.95 hours and the standard deviation was 4.95. Eight of the participants (40\%) previously (or currently) owned an activity tracking wearable device.

Participants were asked whether they had fun and enjoyed their experience playing the games. Figure 4.3 shows the average and standard deviations of the scores for this parameter. ANOVA shows no significant effect for either factor $(F(2,29)=0.07$ with $p=$ 0.93 for exercise, $F(1,29)=1.95$ with $p=0.17$ for device, and $F(1,29)=0.49$ with $p=$ 0.49 for the interaction between them). This outcome can point to the fact that the sense of fun and enjoyment is mostly derived from the actual game content itself (digital aspect) rather than the interaction and communication medium, or even the exercise.

When asked to rate the level of fatigue felt after each game, participants rated rope skipping as the most fatiguing activity among the three exercises. Figure 4.4 illustrates the results ANOVA indicates that the type of exercise has a significant effect at the $p<$ 0.001 level on the feeling of fatigue, with $F(2,29)=10.74$ and $p=0.0003$. The results show that skipping was significantly more fatiguing than running and cycling. There is no significant effect for device $(F(1,29)=0.01, p=0.91)$ and the interaction of exercise and device $(F(1,29)=0.63, p=0.43)$. In general, rope skipping is known to be a highintensity exercise, which can significantly impact the amount of fatigue. The results confirm this notion. As a result, this mode of the game can be used as the difficult level. The wearable devices are both light in terms of weight and do not impact the users' 
performance. It is therefore fair to say that non-intrusive devices such as these are potentially the right choice of device for gamified exercise.

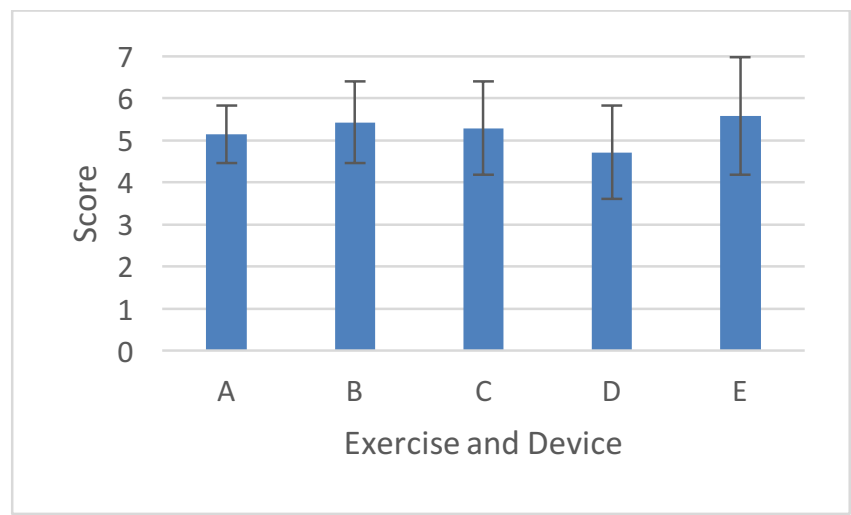

Figure 4.3: User Enjoyment for Different Modes of the Game

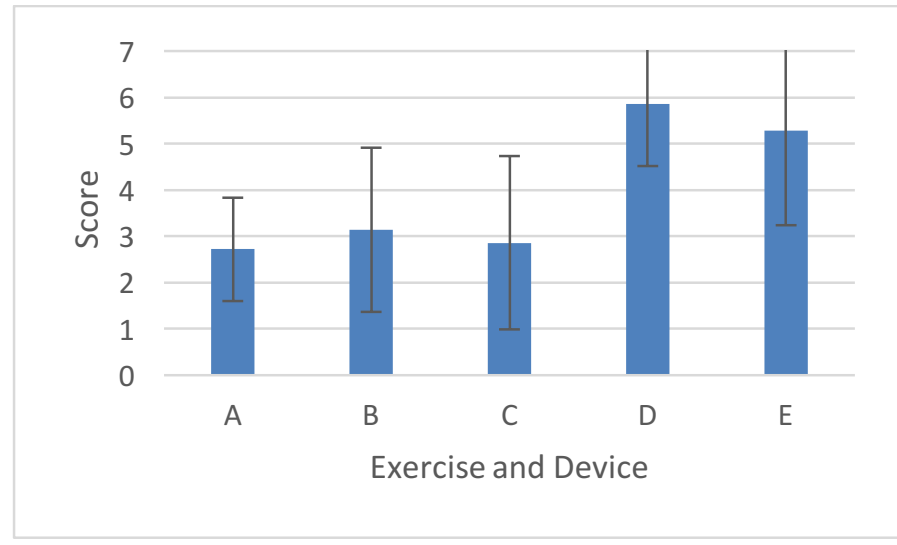

Figure 4.4: Amount of Fatigue for Different Modes of the Game

Following the game session, participants were asked if they felt as if they had exercised/worked out successfully throughout the game. The results are shown in Figure 4.5. There was a significant effect for different devices at the $p<0.05$ level $(F(1,29)=$ $5.57, p=0.02$ ), with the mean score for TI SensorTag being significantly higher than Apple Watch. While the significance of this difference is not particularly strong as per the ANOVA result, we believe that the delay in the Watch could have caused participants 
to perceive a lack of correlation between their exercise and corresponding accomplishments in the game. This could have caused them to believe that it was not them that in fact accomplished the exercise/game goals. In addition, there is no significant effect for exercise $(F(1,29)=0.06$ with $p=0.94)$ and the interaction of exercise and device $(F(1,29)=1.82$ with $p=0.19)$.

When asked whether they found this kind of application motivating for exercise, promising scores were achieved. As shown in Figure 4.6, There was a significant effect for different devices at the $p<0.05$ level $(F(1,29)=5.14, p=0.03)$, with the mean score for TI SensorTag being significantly higher than Apple Watch. This was due to the implementation constraints of the Apple Watch which caused some delay, and the participants were aware of the delay when they were using the system. In addition, there is no significant effect for exercise $(F(1,29)=0.43$ with $p=0.65)$ and the interaction of exercise and device $(F(1,29)=0.82$ with $p=0.37)$.

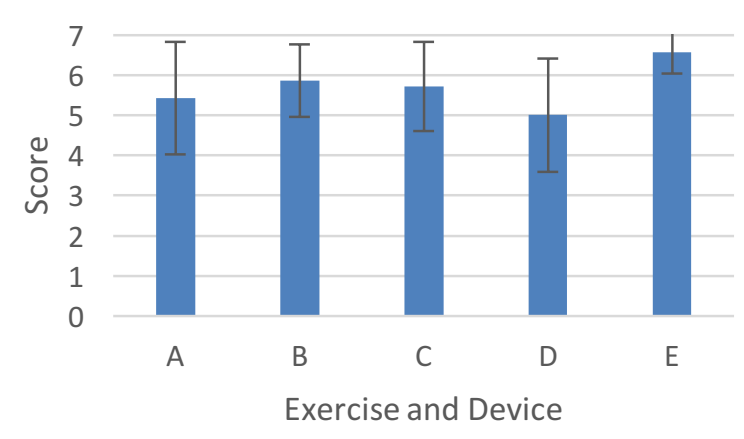

Figure 4.5: Sense of Accomplishment for Different Modes of the Games 


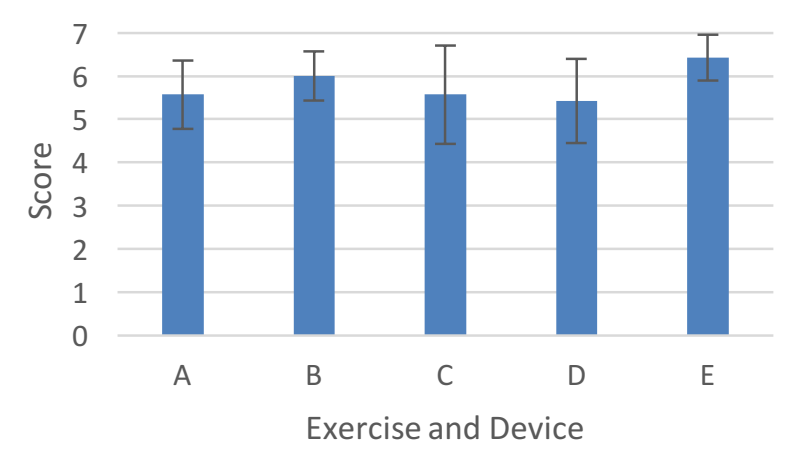

Figure 4.6: Motivation for More Exercise based on Different Modes of the Games

Participants were asked whether they would like to use this application again. The results are presented in Figure 4.7. No significant effect is observed $(F(2,29)=0.19$ and $p=0.83$ for exercise, $F(1,29)=2.22$ with $p=0.15$ for device, and $F(1,29)=0.03$ with $p=0.87$ for the interaction between the two). Similar to the motivational aspects of the application, the results are promising, and users found it useful.

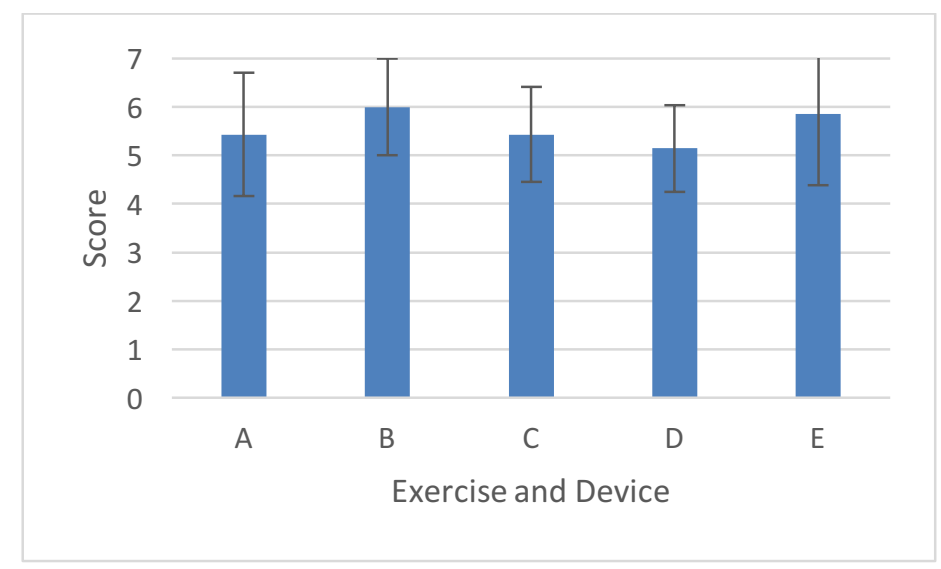

Figure 4.7: Usefulness of the Application based on Different Modes of the Games

Finally, the overall satisfaction and preference of participants was asked. For satisfaction, no significant effect was observed for device $(F(1,29)=2.98, p=0.10)$, exercise $(F(1,29)$ 
$=0.35, p=0.71)$ and the interaction of the two factors $(F(1,29)=0.04, p=0.85)$. The scores for preference were shown in Figure 4.9. The effect of device was significant at the $p<0.05$ level $(F(1,29)=6.45, p=0.02)$ with the SensorTag being higher than the Watch. In addition, there is no other significant effect for exercise $(F(1,29)=0.39, p=$ $0.68)$ and interaction between device and exercise $(F(1,29)=0.13, p=0.72)$.

The absence of a significant effect in satisfaction can be due to the fact that the concept of gamified exercise, especially through wearable devices, is quite novel and attractive for participants. As a result, relatively similar satisfaction scores were observed. Despite this similarity, preference scores were significantly higher for the SensorTag. While this significance was weak, the underlying reasons can be the lag in the Watch, which can make the experience subpar in real-time interactive applications. Software updates that enable access to the inertial measurement unit (IMU) data in real-time can alleviate this issue and increase satisfaction scores.

When asked which device they believed represented their movements more accurately, only $13.55 \%$ of participants chose the Watch, while $86.45 \%$ picked the SensorTag, mentioning the real-time performance as the key reason for their choice. This notion highlights the importance and impact of real-time interaction. While some game designs could be better suited for non-realtime applications (for example games that are based on post-workout leaderboards), we believe realtime systems are more engaging and attractive for gamified exercise. It is therefore important to utilize wearable products that provide real-time access to IMU or other sensor data through their API. 


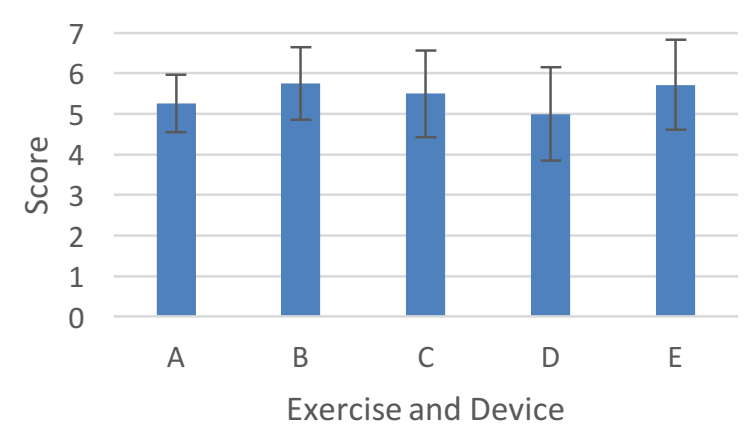

Figure 4.8: Overall Satisfaction of the Different Modes

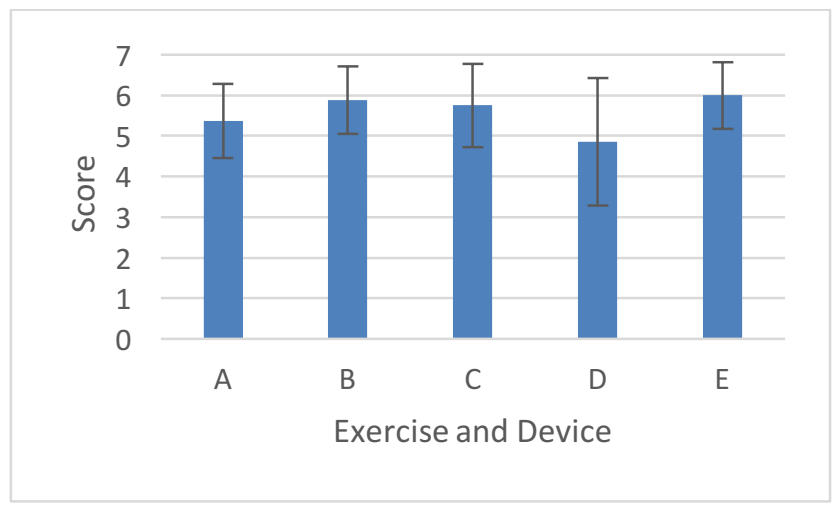

Figure 4.9: Preference Scores for Different Combinations of Exercise and Device

\subsection{Long-term Study}

The second phase of our research included a long-term study of wearable-based exergames with focus on the dynamics of game features and their release as a way to keep players engaged.

\subsubsection{Overview}

Gamification and exergames, in particular have been broadly employed in health and fitness as an attempt to promote exercise and more active lifestyles. Motivated by the popularity and availability of wearable activity trackers, we present the design and 
findings of a study on the motivational effects of using activity tracker-based games to promote daily exercise. Furthermore, we have investigated user behaviors, usage patterns, engagement, and parameters that affect them. An exergame was developed with an accompanying wearable device, for which different variations of application updates were pushed out periodically over a 70-day period. The results of this long-term study show that the usage of wearable activity trackers during exercise, even when gamified for increased entertainment, sees a consistent decline over time. This decline, however, is observed to be reversible with periodic updates to the game. This work, we believe, can make a significant contribution to solving the user retention problem of wearable-based exergames.

\subsubsection{Application Design and Implementation}

StrayBird, the iOS application from our first phase (Zhao, 2016), was used as the basis for this second study for evaluating the long-term effectiveness of the system. The application communicates with wearable activity trackers in real-time and uses data received from these devices as inputs for the game.

All interactions of the new version of the application were same as the one we used in Phase 1. However, a series of new features were added to the original single-user singlelevel game, as shown in Table 4.2. These added game features were designed to provide users with choices of customization, to enhance the real-time game experience, as well as to incorporate social factors. 


\section{Table 4.2: List of Features Added in the New Application}

\begin{tabular}{|c|c|}
\hline $\begin{array}{c}\text { Customization } \\
\text { features }\end{array}$ & Customized bird color \\
\hline Real-time & Customized background color \\
game features & Bird nets (in level 2) \\
\hline \multirow{2}{*}{ Social features } & Thunderstorm (in level 3) \\
\cline { 2 - 2 } & Leaderboard \\
\cline { 2 - 2 } & Achievement \\
\hline
\end{tabular}

The game features a mission-based structure and a point-based system. Higher levels can be unlocked when missions have been achieved. The achievement system is also designed to encourage players during the gameplay. Multiplayer mode brings in leaderboard and challenge boxes that allow players to compete with each other. Figure 4.10 presents several screens from the application. Additionally, the ability to customize the character and background colors were implemented in the application (see Figure 4.11). HockeyApp (Hockeyapp, 2016) was used to distribute different versions of our application to participants, and to receive user feedback.

\subsubsection{User Study Design}

\subsubsection{Participants and Updating Schedule}

The user study was designed to verify the hypothesis that adding different game features and gradually releasing them can positively affect user engagement and retention. 36 participants were recruited from Carleton University by posters for our study. 30 were recruited as main subjects and six as backups who would replace any of the original 30 participants if they quit the study during the 70-day duration of the experiment. The backup participants started and participated in the experiments as others. Each participant 
was provided with a TI Sensortag wearable device (see Figure 4.12) to take home. The wearable could be worn on the wrist or ankle (depending on the exercise they chose; for example, for cycling the sensor would be worn on the ankle). The wearable then connects to the game application that we distributed to the users. No particular set of instructions was provided, and users were allowed to explore the system on their own.

The participants were then randomly divided into three equal groups of 12 (10 as main and two as backup). To users in the first group (referred to as Basic Group), only very basic features of the game such as a single level (level 1) version of the game, as well as single-player mode were provided. Users in the second group (referred to as Full Group) were provided the full version of the application, including the ability to customize colors, as well as all 3 levels of the game, and multiplayer mode with leaderboard and challenge box, etc. Users in the third group (referred to as Updating Group) started with the same application as those in the Basic Group, but periodically received updates (once every 10 days), until after 70 days they ended up with an application that was identical to those in the Full Group. Users were told in the Updating group about receiving updates every 10 days. On each update, notifications were pushed to let them know about the new feature. The different features each group had received are summarized in Table 4.3, and the update schedule for the Updating Group is shown in Table 4.4. 

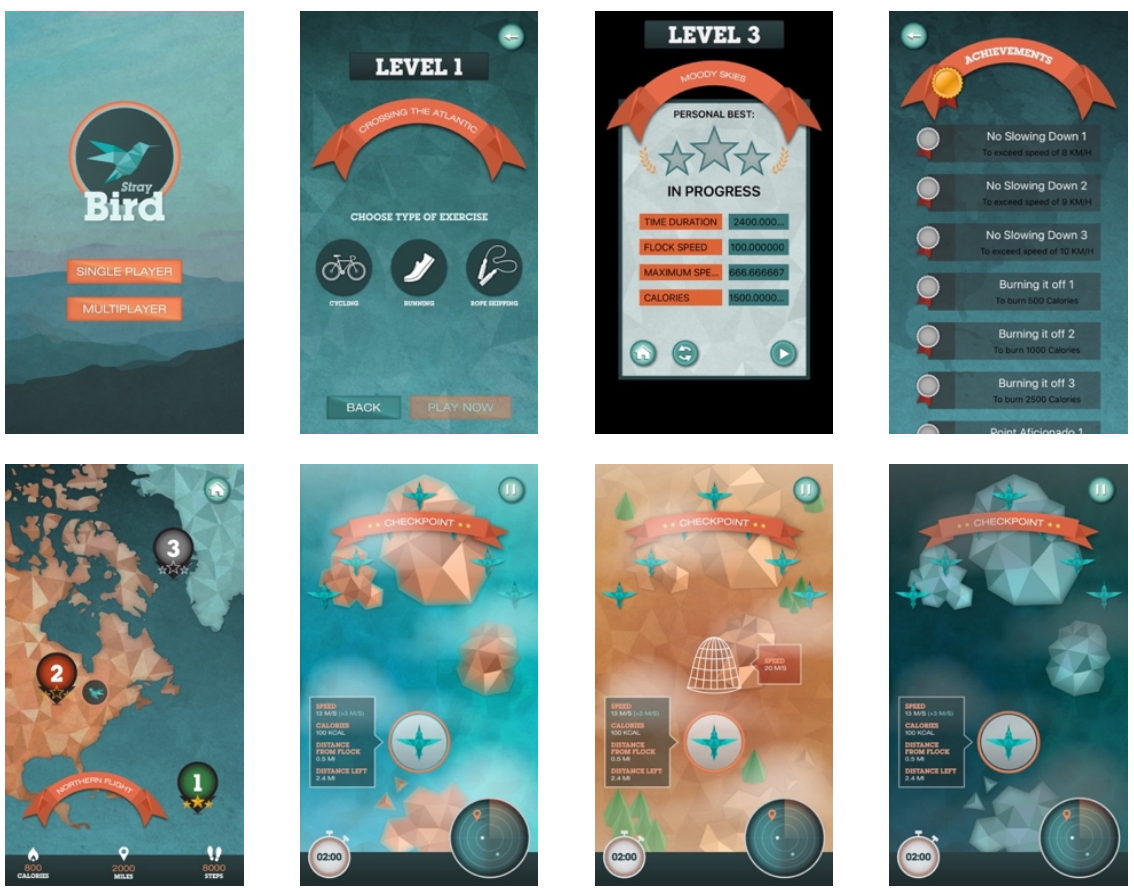

Figure 4.10: Screenshots from Our Original Application. From left to right, row one: choice of player mode, choice of exercises, result screen, achievement screen; row two: landing screen, level 1 view, level 2 view, and level 3 view.
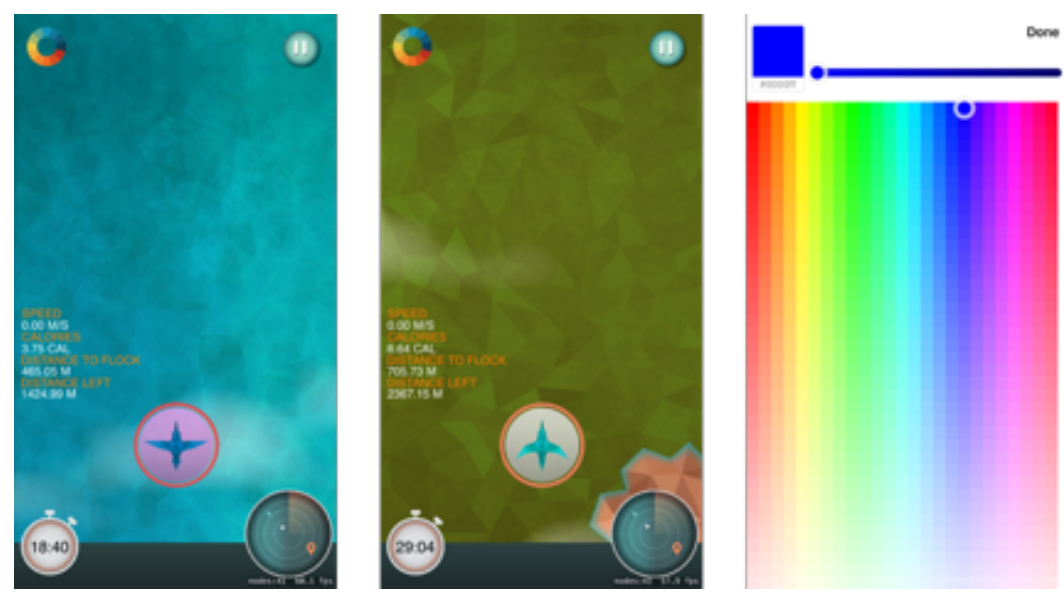

Figure 4.11: Screenshots from Our Updated Application. From left to right: customized bird color for level1, customized background color for level 2 , color picker panel view. 


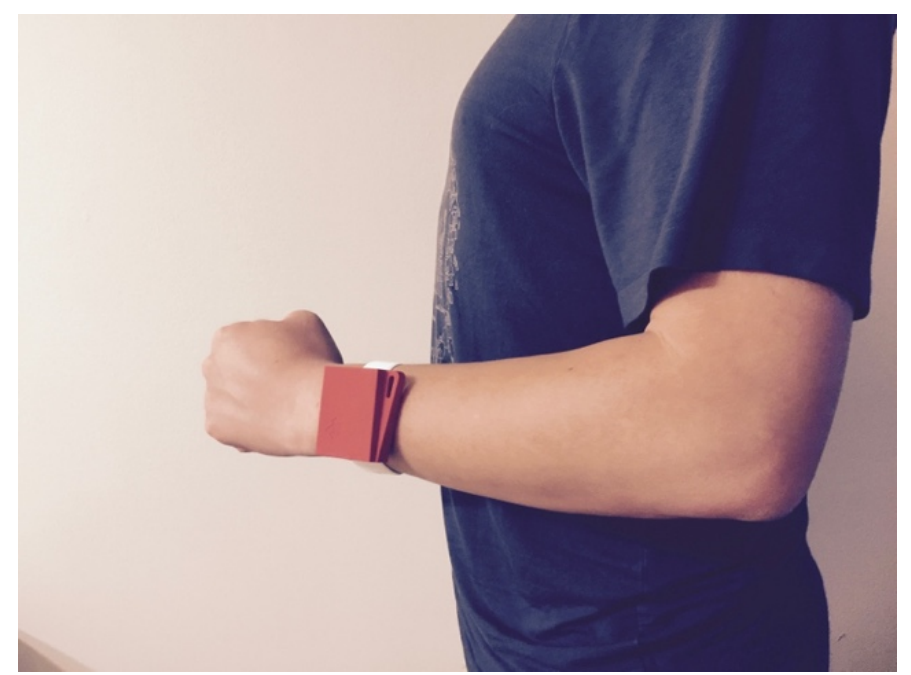

Figure 4.12: The Wearable Device used in Our Study (TI Sensortag)

Table 4.3: List of Different Features

\begin{tabular}{|c|c|}
\hline Basic Group & $\begin{array}{c}\text { Basic features: single level, single } \\
\text { player. }\end{array}$ \\
\hline Full Group & $\begin{array}{c}\text { Basic feature plus all the features } \\
\text { introduced in Table } 1 .\end{array}$ \\
\hline Updating Group & $\begin{array}{c}\text { Start with basic features (same as } \\
\text { the Basic Group), with other } \\
\text { features gradually added (as } \\
\text { described in Table 3). }\end{array}$ \\
\hline
\end{tabular}

Table 4.4: Automatic Updates Schedule used for the Updating Group

\begin{tabular}{|c|c|}
\hline Day & Updates \\
\hline Day 1 & Single level, single player \\
\hline Day 11 & Add customized bird color \\
\hline Day 21 & $\begin{array}{l}\text { Add multiplayer leaderboard and } \\
\text { challenge-box }\end{array}$ \\
\hline Day 31 & Add level 2 (with bird net) \\
\hline Day 41 & Add customized background color \\
\hline Day 51 & Add achievements \\
\hline Day 61 & Add level 3 (with thunderstorm) \\
\hline
\end{tabular}




\subsubsection{Procedure and Data Collection}

Pre-study

Participants were asked to complete questionnaires upon receiving and returning the wearable device. The pre-study questionnaire asked demographic questions including age, gender, height, weight, the hours they spend per week in exercising, any wearables owned, and the types and duration of video games (PC, console, and mobile) played.

\section{In-game data}

For in-game data collection, the Google Analytics API (Analytics, 2016) was used to track users' comprehensive behavior data, including screen views and tapped events with the associated timestamp, exercise sessions, performance, scores, and feedback and comments. Users were also asked about their level of encouragement through a 5-point Likert question (from $1=$ highly discouraged to $5=$ highly encouraged) after each completed session they have exercised.

\section{Post-study}

A post-study questionnaire was conducted to comprehensively evaluate participants' experience during the study. Firstly, participants were asked three general questions to measure their overall motivation, satisfaction, and preference based on their experience with the game:

- I find this kind of application motivation for exercise;

- I was overall satisfied with this application; 
- I prefer using this type of application for exercise over regular exercise.

Parts of Intrinsic Motivation Inventory (IMI) (Imi, 2016) measurement was included to assess participants' subjective experience related to the game experience. The detail questions are attached in the Appendix for reference.

In the post-study questionnaire, participants used a 7-point Likert scale (from $1=$ strongly disagree to $7=$ strongly agree) to rate the game-play experience, followed by some openended questions. Some open-ended questions were also included regarding their preferred exercise and game mode, suggestions on both software and hardware of the system, as well as their opinion on how the game could be more engaging based on their game experience.

Ethics approval was received from the Carleton University Ethics committee. Participants received a $\$ 10$ honorarium gift card to thank them for their participation in the study. During the 70 days of the user study, 4 out of the 30 participants withdrew for different reasons, and 4 participants from the back-ups of the same study group were randomly selected to replace the original participants.

\subsubsection{Results and Analysis}

\subsubsection{Demographic Information}

Out of the 30 volunteers who participated in this study, 19 were males and 11 were females. Their average age was 24.71 years with a standard deviation of 4.23 years. Their self-reported average hours of exercise per week was 3.81 hours with the a standard deviation of 2.83 hours, while the self-reported average hours per week spent on playing 
games was 6.33 for pc/console games (with a standard deviation of 9.51) along with a 4.73 hour per week spent on playing smart-phone games (with a standard deviation of 6.33). 11 out of 30 participants (36.7\%) previously (or currently) owned an activity tracking wearable device.

\subsubsection{In-game Data}

Figure 4.13 shows the participants' choice of exercise among the three classes enabled during our study (from in-game data monitoring), where running is the most popular, followed by cycling. This result matches the subjective results we collected from the post-study questionnaire, where the vast majority preferred running as well. Figure 4.14 shows the feedback that we collected during the study (after each completed session) using a 5-point Likert scale (from $1=$ highly discouraged to $5=$ highly encouraged) for regarding the level of encouragement by the system towards more exercise. A total of 322 answers were collected, 47 from the Basic Group, 105 from the Full Group and 170

from the Updating Group. We can see that most of the users felt positively regarding this gamified exercise experience. The Full Group felt most encouraged (with $82 \%$ of participants choosing options higher than "no difference", followed closely by the Updating (80\%) and Basic Groups (70\%).

\subsubsection{Overall Motivation, Satisfaction and Preference}

A one-way between-groups analysis of variances (ANOVA) and Post-Hoc TukeyKramer HSD test (Jaccard, 1984) was conducted to analyze the main effects between the three groups. The alpha was set at 0.05 for all statistical tests. 


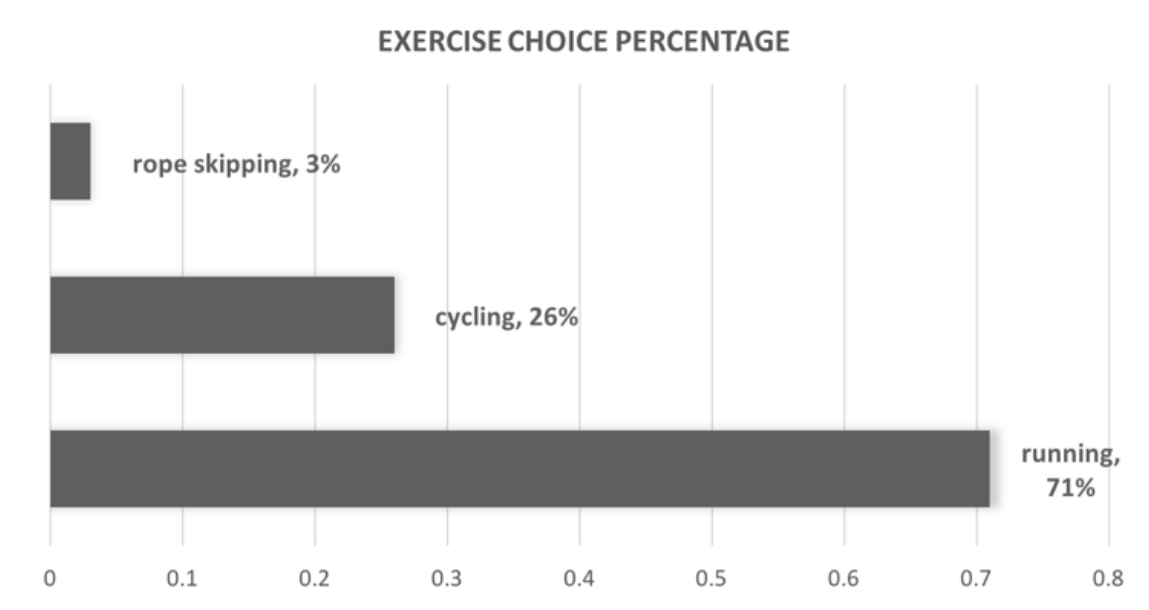

Figure 4.13: Choice of Exercise (from in-game data monitoring)

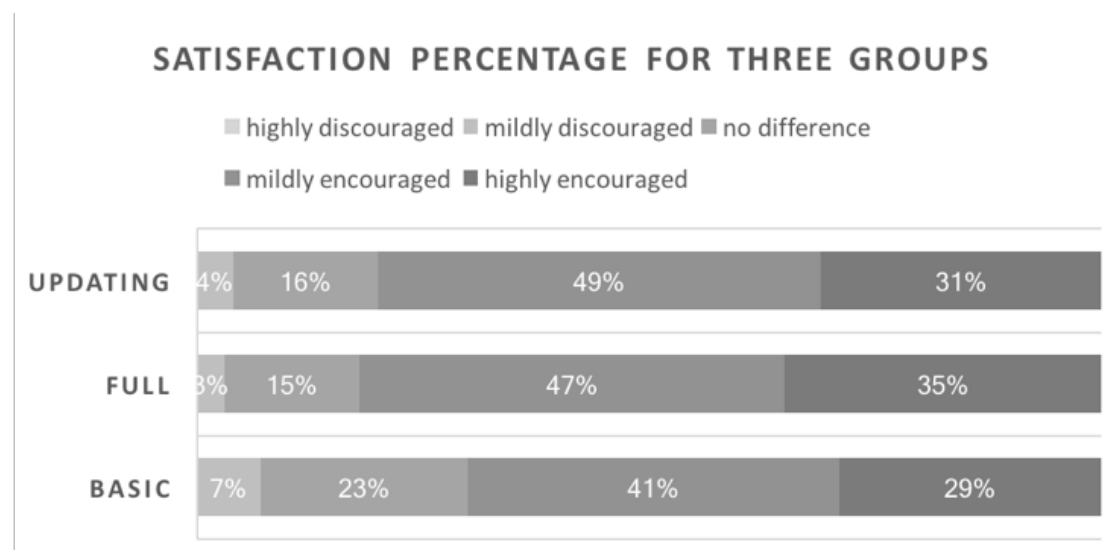

Figure 4.14: Level of Encouragement (asked after each completed workout session)

Figure 4.15 shows the average and standard deviations of the scores for question 1 to 3 . Please note that the subscript B represents the Basic Group, F represents the Full Group and U represents the Updating Group. The detailed Tukey-Kramer HSD post-hoc test results for significant factors are shown in (Zhao, 2017) and the pairwise comparison significance are also marked in Figure 4.15.

From the results, we can see that the Full Group and the Updating Group show significantly higher motivation and satisfaction compared to the Basic Group. This 
suggests that the number and types of features are the main factors affecting overall user motivation and satisfaction. No significant effect in preference over regular exercise, suggests that all participants, even in the Basic group, still prefer using the gamified exercise experience over regular exercise.

\subsubsection{Feature Importance Level}

Only the Full Group and the Updating Group were asked to answer the second section of the questionnaire regarding the different game features (the Basic Group had only received a basic version of the game without any features for evaluation). We used statements such as: "Leaderboard was important in my game experience." To evaluate the importance level of each separate game feature. The results are shown in Figure 4.16, in which feature 1 to 7 represent: 1: Customized bird color; 2: Customized background color; 3: Bird nets (in level 2); 4: Thunderstorm (in level 3); 5: Leaderboard; 6:

Achievements; 7: Challenge box.
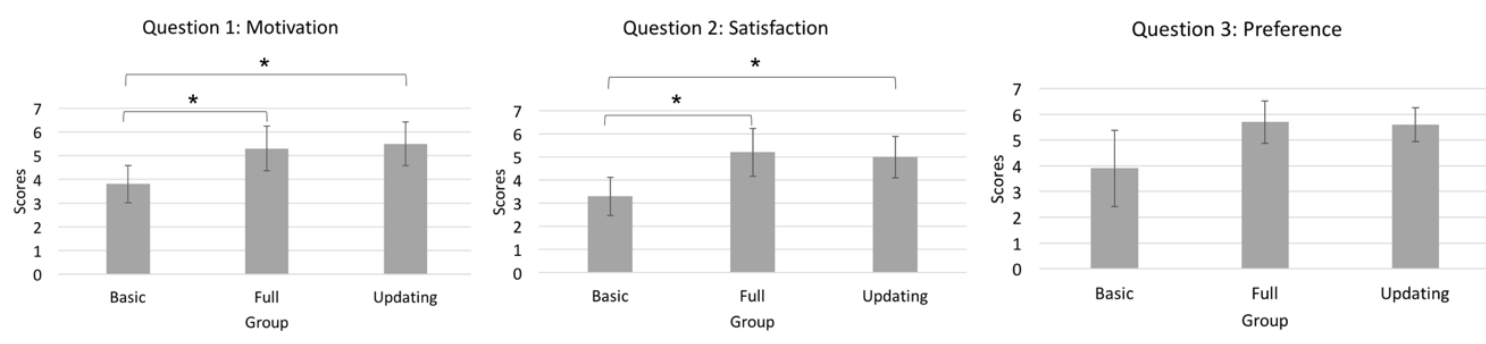

Figure 4.15: Result for Post-study Question 1, 2 and 3. From left to right: overall motivation, overall satisfaction, and overall preference over regular exercise

Results on the importance level of different game features show that social factors including Leaderboard and Achievements play important roles in-game experience. However, also being one of the social factors, the challenge box is considered less 
important. A possible reason leading to this result could be the lack of real-life relationships between our participants. We believe a closer relationship between users could strengthen the importance level of the challenge box. We did not add invitation feature to this study since we need to keep a consistent user group, but adding such ability in the future can potentially add to the social value. Also, it is found that the scores of Full Group are relatively higher than the Updating group in most of the game features except for customized bird color. This may be due to the customized bird color was the first updating feature that the Updating Group had received, so that it became more impressive compared to other features, although this is not conclusive.

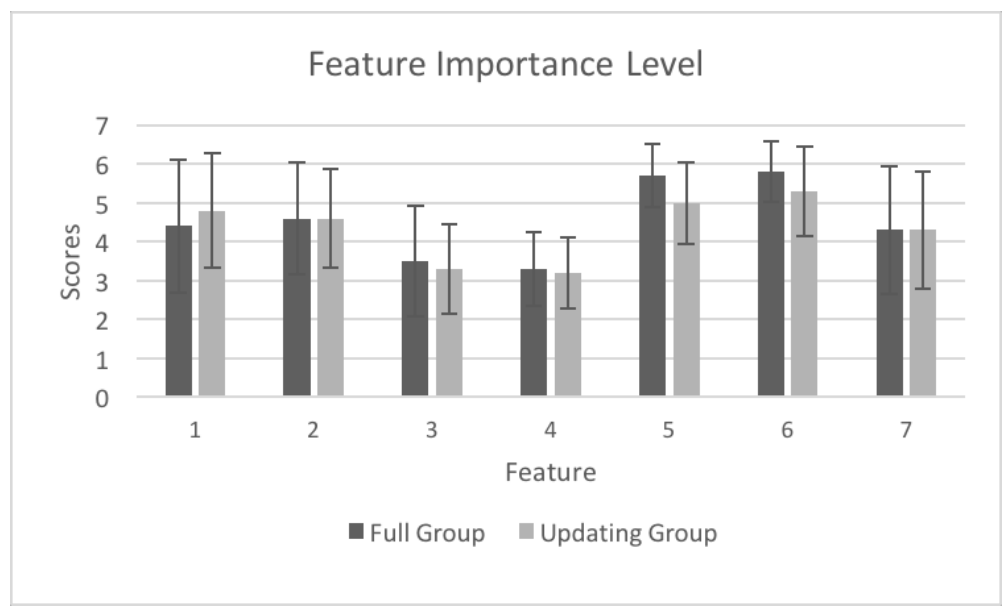

Figure 4.16: Impact of Different Features for the Full and the Updating Groups

\subsubsection{IMI Sub-scales}

A reliability analysis is conducted because we modified the original IMI scale to better fit the concept of the game. A Cronbach's alpha value of $0.878(\alpha=0.878)$ is obtained (a 
commonly accepted rule is that an alpha of 0.7 indicates acceptable reliability and 0.8 or higher indicates good reliability. (Tavakol, 2011))

Figure 4.17 summarizes the result of IMI sub-scales. We can see the number and diversity of game features could impact user motivation towards the gamified exercise system, since there are significant effects between the Basic Group and the Full Group, as well as between the Basic Group and the Updating Group. On the other hand, gradual updating does not take away from motivation, since there is no significant effect between the Full Group and the Updating Group. This indicates that by pushing out gradual updates, we can benefit from the advantages of the approach, namely increased engagement and retention, while not negatively impact motivation.

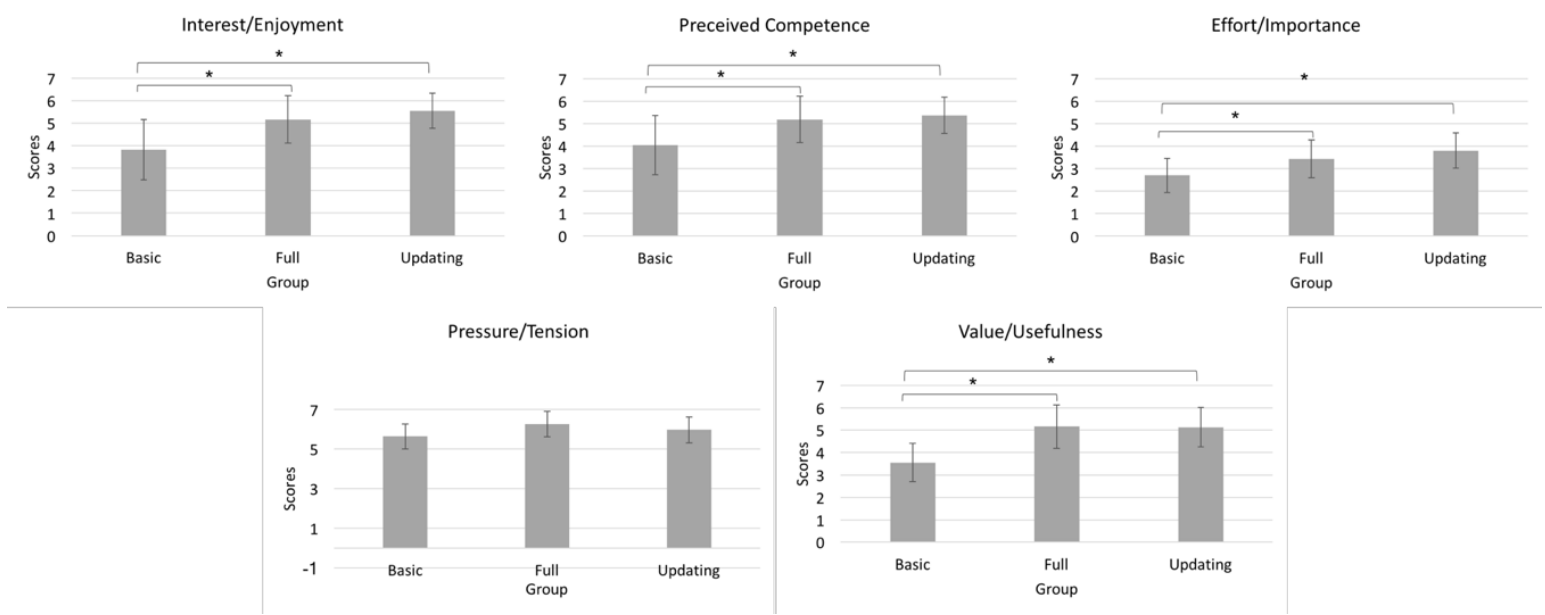

Figure 4.17: Average and Standard Deviation of IMI: Interest/Enjoyment, Perceived Competence, Effort/Importance, Pressure/Tension, and Value/Usefulness.

\subsubsection{Daily Active Users and Workout Sessions}

Figure 4.18 shows the change of active users of all three groups during the 70 days. We can see for active users, there is an overall descent trend that appears as time grows for all 
three groups, while the Basic Group declines the fastest. The waves of the Full Group were basically caused by weekends. The large variance in the Updating Group was caused by the regular updating plus the weekend. Figure 4.19 shows the accumulated activate users for three groups with a regression line. Figure 4.20 shows the total started workout sessions and complete sessions of all three groups, from which we noticed a basically same trend as the active users. The decline of the Basic Group is the fastest, and the Full Group is mostly affected by weekends when people have more time for exercise, as shown in Figure 4.21, the participants' overall completed sessions by the time of day. The Updating Group maintains a relatively higher value both in started and completed sessions, which indicates the gradually updating not only brings back users to check the updates but actually promotes engagement towards the actual behavior of exercise. Figure 4.22 shows the accumulated completed sessions with the regression line. Compared to Figure 4.19, we found the accumulated complete sessions are overall rising slower compared to active users. This indicates there are circumstances that participants only either open the application to check updates or play around with it, without actually accomplishing any exercise session. This made us realize that despite the elements we considered important regarding exercise experience, other features that not directly linked to gameplay may also affect user retention and the application's life cycle.

Users received notifications (through Apple Push Notification service) when updates were available for the application. It was observed that many users returned to the application to check the updates. This contributed to the number of completed workout sessions being increased. Since the Updating Group contains the most active users and completed sessions, we can conclude that the gradual releasing of updates can not only 
attract users to interact more with the application, but also motivate them to complete gamified exercise sessions. The total in-game workout time of the Basic Group was 439 minutes (4.3 minutes per session on average), the total time for the Full Group was 1073 minutes (an average 6.1 minutes per session), and for the Updating Group, the total was 1322 minutes (an average of 5.7 minutes per session). The reason that the average time for the Updating Group is slightly lower than the Full Group is that we released the second and third level at the beginning to the Full Group but in the middle of the study to the Updating Group, and higher levels are longer in duration. We can see that both in Figure 4.19 and Figure 4.21, at the start of the study, the Full Group accumulated more active users and completed sessions, but roughly after the second update, the accumulated numbers of the Updating Group exceeded the Full Group and kept a higher slope till the end of the study. We can conclude that the user's curiosity towards new features can make the game more sustainable over the long run, resulting in a relatively higher user retention rate.

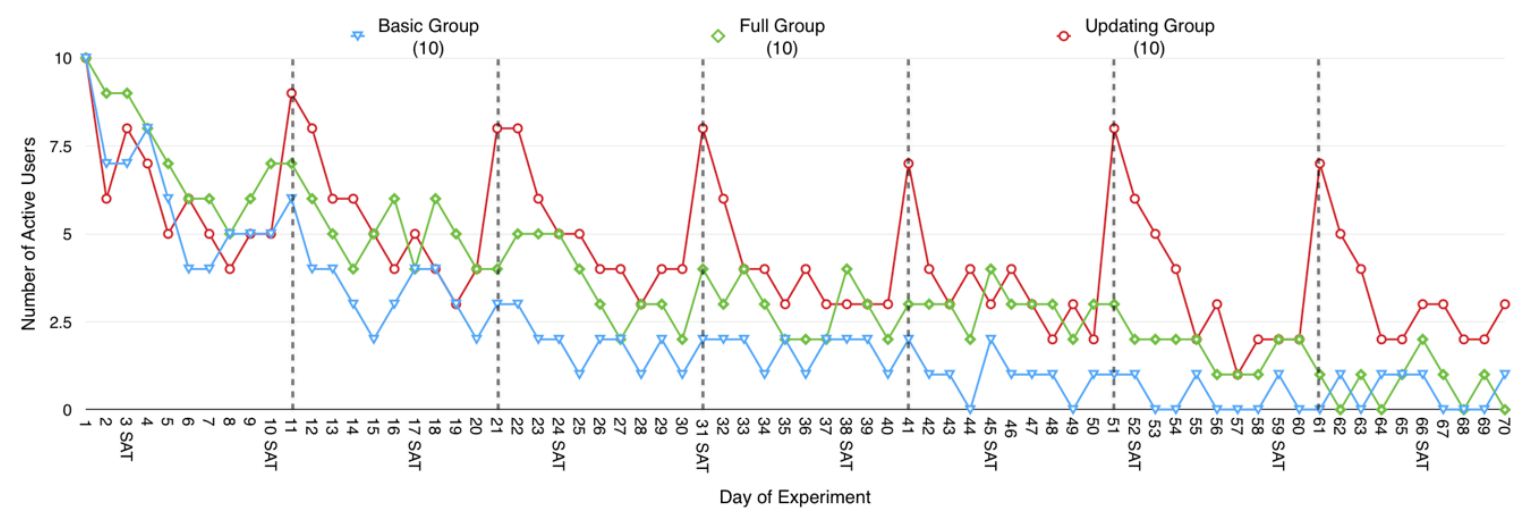

Figure 4.18: Number of Active Users per Day (dotted vertical lines indicate the application update days) 


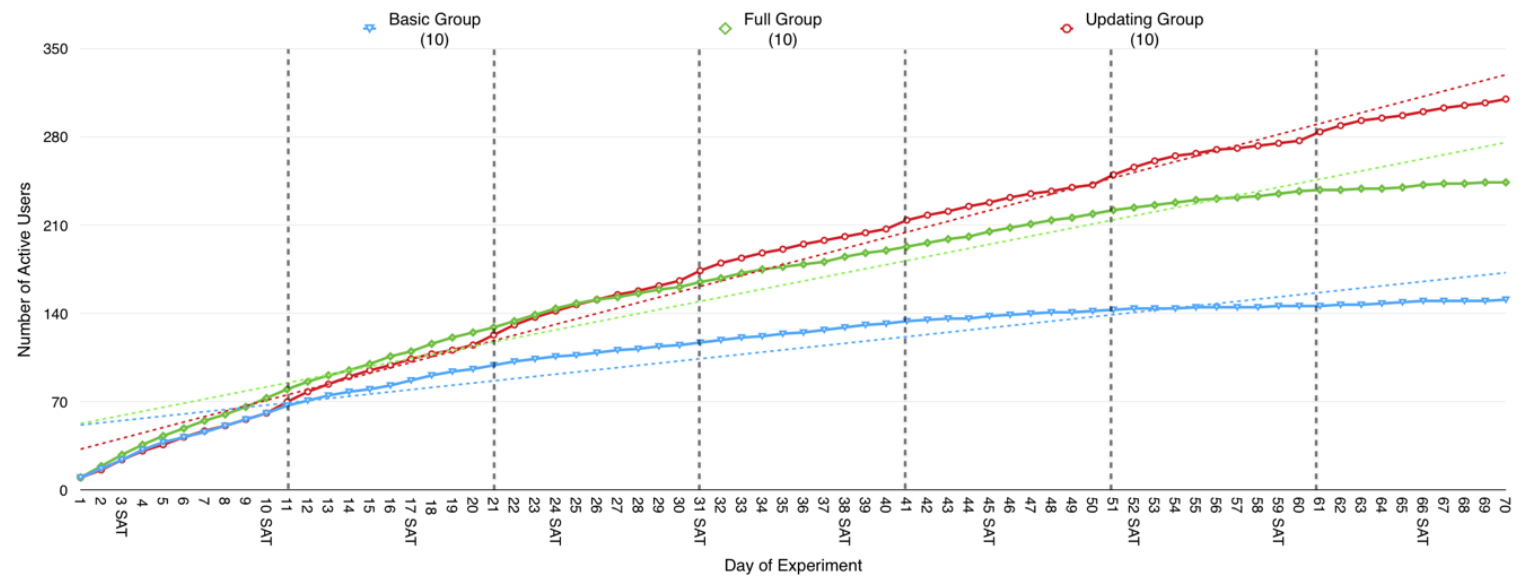

Figure 4.19: Accumulated Active Users for all 3 groups with the regression line

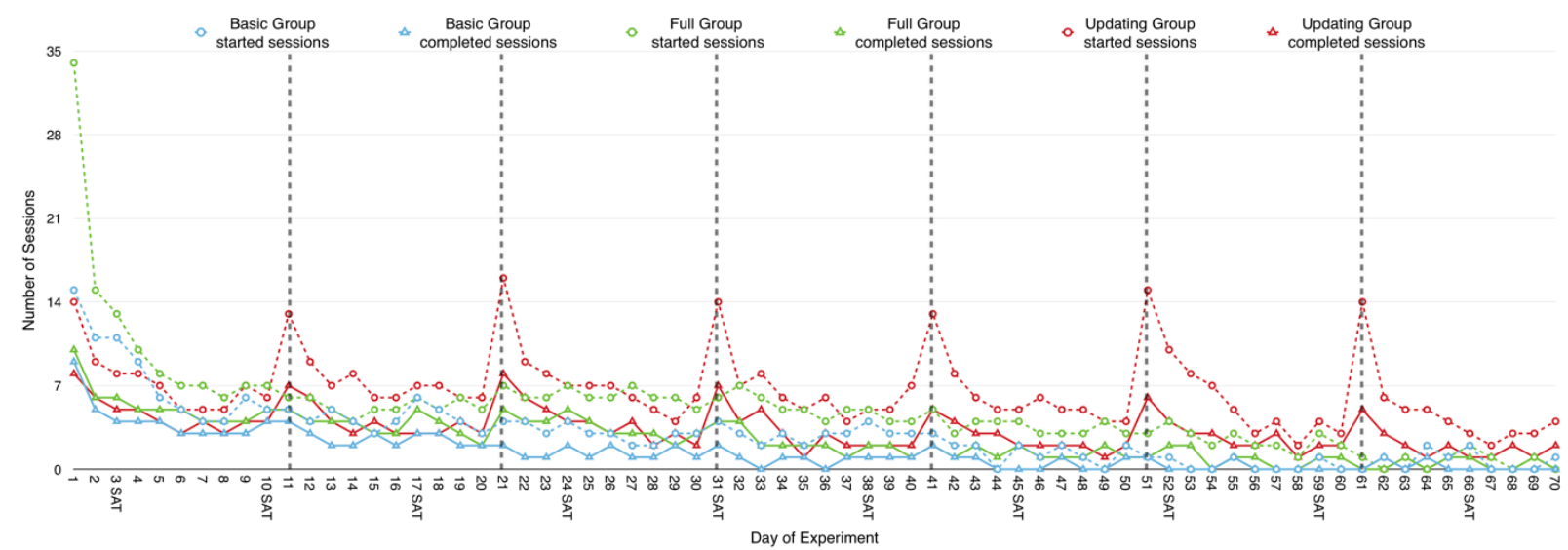

Figure 4.20: Number of Started and Completed Sessions per Day (dotted vertical lines indicate the application update days)

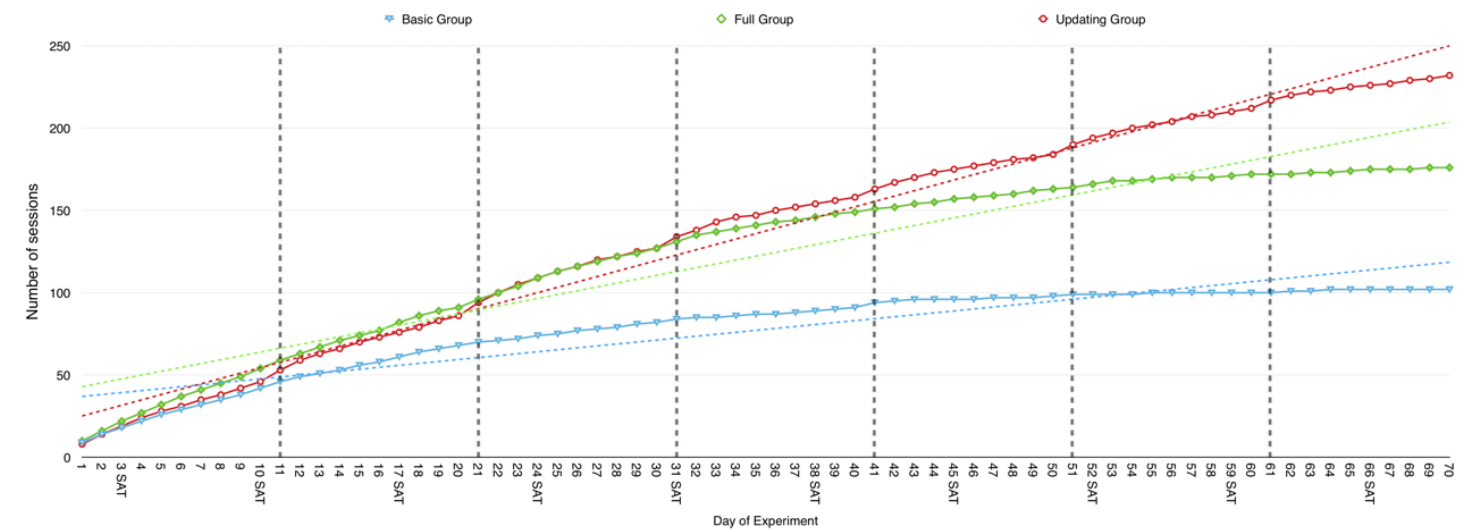

Figure 4.21: Accumulated Completed Sessions with the regression line 


\subsubsection{Discussion}

Overall, in this 70-day user study, the evaluation on motivation showed that more and diverse features could increase user motivation toward the gamified exercise system, and the gradual release of game features does not negatively affect user motivation. The analysis on game sustainability showed that while there was a decline in activity level in all groups, the gradual release of features improved the sustainability to a certain extent which verifies our hypothesis that various game features and the way they are released will influence user retention.

While the Basic and Full feature groups started to asymptotically level off within the 10week study (in weeks 5 and 9 respectively), this was not apparent in the Updating group (see Figure 4.21). This suggests that minor feature updates can have the effect of increasing sustainability over the long term. We further believe that a combination of minor and major updates will likely have an even longer effect on sustainability.

It can be seen in Figure 4.20 that sometimes the notification of new features only brings people back to check updates but without completing workouts. This increases the number of minutes of engagement with the game, not the number of actual exercises. One of the participants indicated that changes, such as customized color, did not create enough interest to complete a session. However, addition of the leaderboard significantly increased the motivation to complete sessions with improved results. Accordingly, we can see that different users were motivated by different features, and this can be considered as the main cause of some incomplete sessions. Player modeling techniques can determine the types of features and updates that can motivate different users. 
Therefore, customized feature updates may help improve workout completion rates, which is part of our future work.

The results of this study also show that participants in all groups preferred the gamified exercise experience over regular exercises; features related to social factors played a relatively more important role in this game experience, followed by customization features.

There are some limitations regarding this work. One limitation is with respect to participant characteristics. The participants consisted only of university students, which limits the generalizability of the results. Future research may replicate this study by recruiting participants of various age groups, gamer types (e.g.: gamer vs. non-gamers) and people with different exercise goals (e.g.: leisure vs. fitness). People of different ages or who have different exercise goals could have different motivations for using the system. It is important to understand what these goals are and continue to redesign and redevelop game features to keep players engaged in the game and exercise. Another limitation of this study was data collection. We used Google Analytics in this work, which contains less than $1.5 \%$ missing data. While this is considered acceptable by most standards (Blast, 2013), the accuracy of the results is reduced nonetheless. Thus, a future study could employ more accurate analytical tools/software for obtaining better results. 


\section{Chapter 5: Personalized Exergame (Research Phases 3 and 4)}

\subsection{Personalized Fitness Assistant System with Player Modeling}

\subsubsection{Overview}

Persuasive Games and gamified systems (Fogg, 1999) are effective approaches for motivating human behavior. While recent years have seen an increase in persuasive applications designed for promoting more active lifestyles (Consolvo, 2009), studies have suggested that the "one-size-fits-all" approach does not work for such persuasive applications (Orji, 2013). There is an increasing demand for personalization as a means of tailoring an experience to individual needs and interests (Orji, 2017). This is particularly the case for persuasive and recommender systems such as those in marketing, education, and health (Orji, 2014). Among different personalization solutions, player modeling in games that aims at understanding players to enhance the game experience has been a core research topic within the Artificial Intelligence (AI) field (Charles, 2013). It aims to describe a game player's traits and preferences, as well as the players' cognitive, affective and behavioral patterns (Yannakakis, 2013) within well-defined structures that allow designers to tailor game content or goals automatically to suit the needs or preferences of an individual player.

Meanwhile, Gamification, the idea of utilizing game thinking and game mechanics in non-game contexts (Deterding, 2011), has rapidly emerged over the past years, especially in the area of exercise and fitness, as a tool to promote health and active lifestyle (Wind, 2001). It has the potential of helping users achieve their fitness goals and increase engagement and pleasure by adding game features to physical activity. 
However, most existing work on gamified and persuasive games in health and wellness are limited due to the use of a one-size-fits-all approach, which has been shown to be suboptimal (Rimer, 2006). Initial attempts at personalization are mostly limited to a small set of static parameters about the user (such as age, gender, and occupation) which makes their "recommendations" static, less effective and personalized. Also, their effectiveness at promoting the desired behavior was mostly evaluated based on a single point of use and feedback (short-term). To address this gap, the study presented in this paper aims to look at the long-term effectiveness of a personalized gamified fitness assistant system that gradually establishes an individual player model (for each unique user) over a relatively long period (60 days).

To address the shortcomings, our research at this phase aims to answer the following questions (RQ3 and RQ4):

1. Is it feasible to provide participants with personalized activity recommendations by using continuous player modeling?

2. Can player modeling and gamification techniques improve user engagement/experience towards fitness activities over time?

Specifically, we present a gamified 24/7 personal fitness assistant system which 1) tracks, recognizes and predicts user's daily activity pattern dynamically, 2) provides personalized recommendations on certain types of exercise (taking into consideration the proper time, location, the type of exercise and intensity, etc.,) and 3) generates personalized gamified experience for individual user. 
Forty-eight participants were recruited for this study ( 40 were recruited as main subjects and eight as backups). We divided our participants into four groups: The Full group received the application with both gamified features and personalized recommendation, the Personalized group received only personalized recommendation but no gamified features, the Gamified group received non-personalized recommendation with gamified features added, and the Control group received non-personalized recommendations and non-gamified features. Our gamified application is a conversation-based app, in which the user talked to the "future him/herself", who will guide the user through daily activities which are generated by the recommendation system, in a gamified structure. The behavioral patterns of all four groups were monitored by tracking participants' activities as well as collecting their subjective experiences through questionnaires.

The results of the study running for 60 days shows that the idea of generating personalized exercise recommendations using player modeling is feasible. Above all, it shows that personalizing recommendations using player modeling and gamification improve participants' engagement and motivation towards fitness activities over time.

Our work offers three major contributions: 1) it presents a gamified fitness assistant system that uses player modeling to recommend personalized activities based on various personal parameters, 2) it shows the effectiveness of combined gamification and player modeling for personalization through a 60 -day long-term study with 40 participants, and 3) we propose and show the effectiveness of continuous activity tracking and player modeling vs. more common static and one-time modeling. 


\subsubsection{Methodology}

\subsubsection{System Design}

A gamified 24/7 fitness assistant system was designed and implemented for this study. The system architecture is shown in Figure 5.1 below.

The system contains mainly three components: a player model, a recommendation engine, and a game generator. A Wear OS (formerly Android Wear) application was implemented as the user interface which tracks user's activities and provides gamified fitness recommendations. Pre-study questionnaires were conducted to collect user's demographical information, as well as to determine their gamer and exerciser types.

The player model consists of four sub-models: 1) an activity recognition model, 2) a general model, 3) an exerciser type model, and 4) a player type model. Each of the submodels is mainly in charge of generating one part of the recommendation as shown in Table 5.1 below.

Table 5.1: Roles of each sub-model

\begin{tabular}{|c|c|}
\hline Sub-Model & Role \\
\hline Activity recognition model & Time/location \\
\hline General model & Intensity/duration \\
\hline Exerciser type model & Exercise type \\
\hline Player type model & Game elements \\
\hline
\end{tabular}

Although each sub-model is designed for generating one particular part of the recommendation, they are still connected to each other for creating a more reliable overall recommendation. For example, the Exerciser type model is built for each 
individual user, for recommending different types of activities based on their personalities, but it also relies on the General model, which is built based on the user's fitness and health condition, for excluding those activities that may be suitable for their personality type, but not for their health condition. We referred to the theoretical foundations from the Global Recommendations on Physical Activity for Health (GRPAH) (WHO, 2010) to determine proper exercise recommendations. The GRPAH is an accepted tool, approved by the World Health Organization (WHO) for recommending appropriate exercise type, duration, and intensity. The player type sub-model is not connecting to other sub-models, because it only controls the game elements but not the activity recommendations.

The Recommendation Engine is a decision tree-based module that uses all the information generated from the player model to create personalized recommendations for each individual user. It could either extend an existing activity (for example by recommending a longer exercise time, a longer running path, or appropriate intensity), or recommend some activities on the user's idle time, or just recommend a different type of activity.

The Game Generator is responsible for adding game elements to the recommendations to gamify the activity suggestions generated by the recommendation engine. The type of game elements to be added is determined by the Hexad player type model. Details of the game and activity recommendation are provided in section 5.1.3.2. 


\subsubsection{Application Design and Implementation}

A WearOS application was developed for this study. The application is a conversationbased game whereby all the interaction happens in the form of a conversation between the user and the "future him/herself". The game is based on a story that one day user received a message from the future him/herself in 20 years, telling him/her that the world is about to end in that future world, but only the user can save it by completing a series of tasks. Then the future self will guide the user through daily activities which are generated by the recommendation system, in a gamified structure. The main user interface and the application icons are shown in Figure 5.2 below.

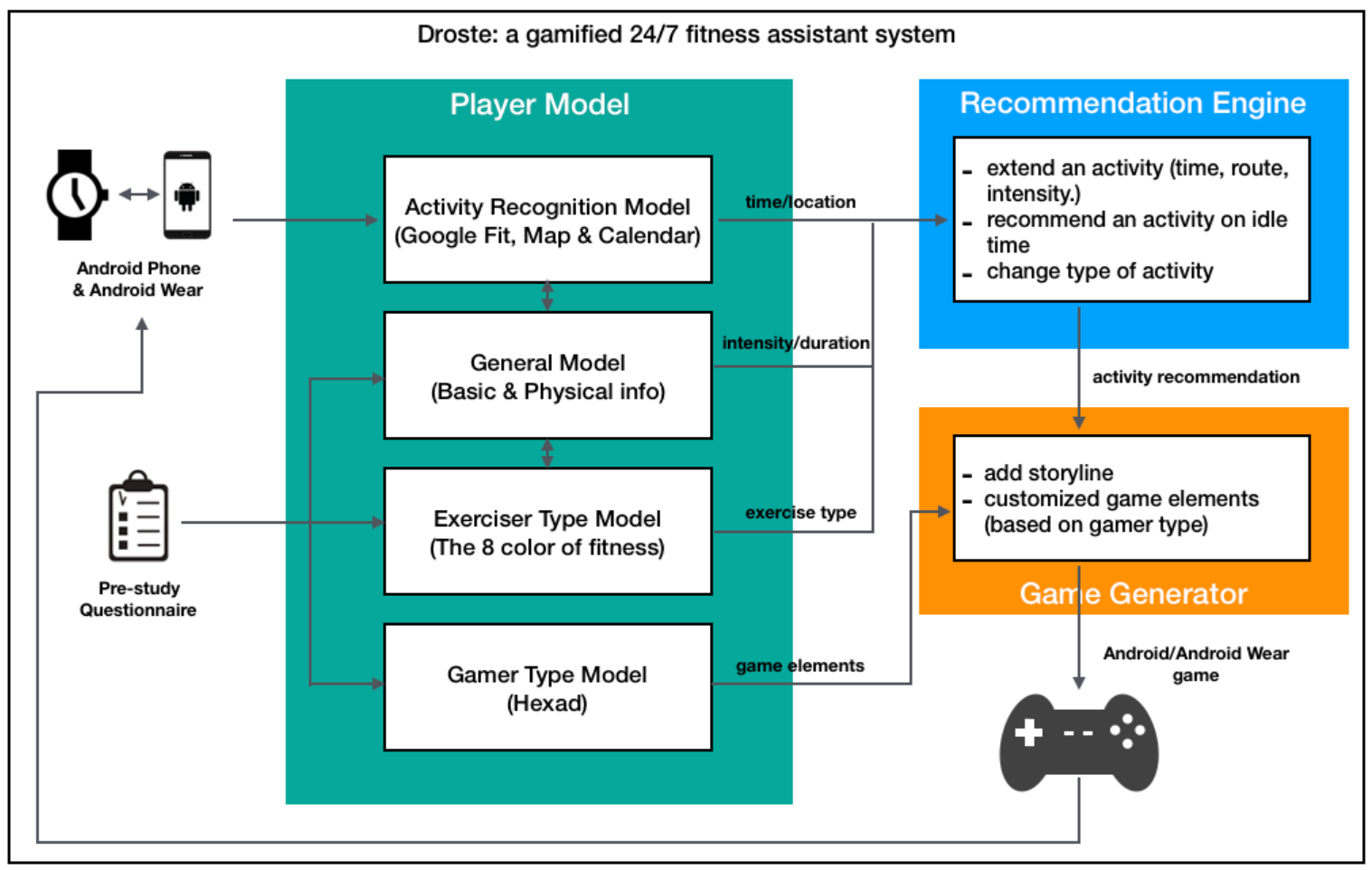

Figure 5.1: System architecture 

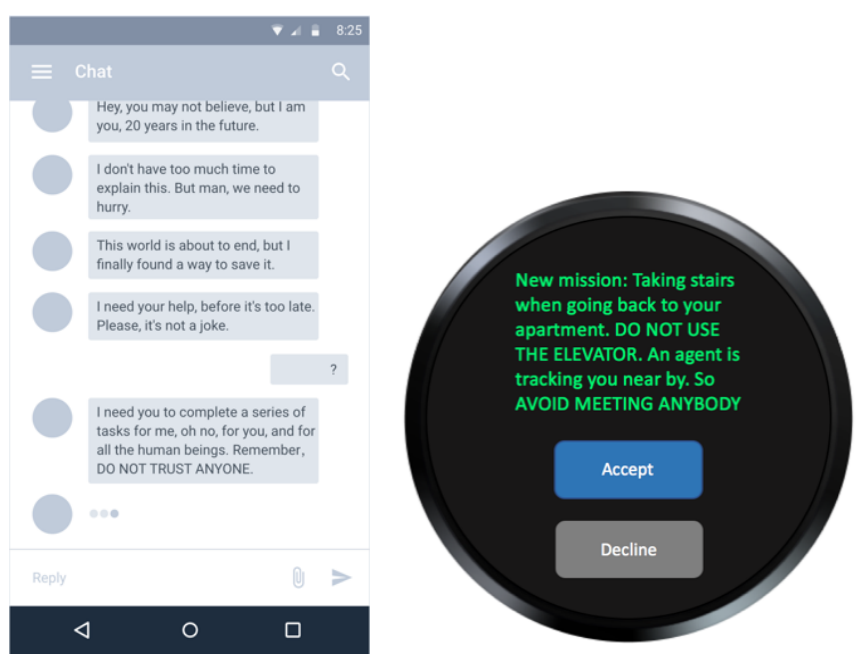

Figure 5.2: Example of application interface and icon. Left: Android UI, right: Wear OS UI

The application tracks the user's daily activity through Android ActivityRecognition (ActivityRecognition, 2018) and Google Fit Application Programming Interface (API) (GoogleFit, 2018) which allows up to six user activities to be recognized in real-time: in vehicle, on foot, running, walking, on bicycle, and standing still.

The Google Fit API provides encapsulated daily activity-related data such as calorie consumption, daily steps, as well as heart rate history (if applicable) tracked by both phone and watch sensors. All these activity data, along with their time stamps and location information, were used as input features to train a daily activity model for each individual user, by which possible exercise time and location were predicted. As shown in Figure 5.2, the application is a conversation-based game. We used Wit.ai (Wit, 2018) to generate storylines and to build a bot that can talk to participants. Wit.ai is a tool that uses NLP (Natural Language Processing) to understand human language, and we used its /message API to create a Chabot, which aims to understand user's intents and lead participants to designed storylines. Moreover, we also included a weather assistant in the 
system (through Weather.com API (Weather, 2018)) to help participants in planning activities around the weather. MongoDB Atlas (Atlas, 2019), which is a cloud-based database service by MongoDB (https://www.mongodb.com/cloud/atlas), was used as the database to store the modelling data. We then used it in Microsoft Azure Machine Learning Studio to further train the PA prediction model in research phase four.

When designing the game features, we employed the Hexad player types and Game Design Elements guide (Nacke, 2014). It suggested which game design elements are preferred by each player type, and we implemented one element for each type of user in this study, for personalized game experience (in addition to the game storyline). The game elements added for each player type are shown in Table 5.2 below, and Figure 5.3 shows some screenshots of example game elements for different player types.

Table 5.2: Different game elements added for each type of player

\begin{tabular}{c|c} 
Player Types & Game Elements \\
\hline Achiever & Challenge \\
\hline Player & Points \\
\hline Socializer & Link to social network \\
\hline Philanthropist & Game experience sharing \\
\hline Disruptor & Hacking mode \\
\hline Free Spirit & $\begin{array}{c}\text { Theme color } \\
\text { customization }\end{array}$ \\
\hline
\end{tabular}

We used The 8 Colors of Fitness model (Brue, 2008) for suggesting different types of activities. This model is one of the few that use personality type as the basis of activity recommendation and is suggested by other researchers and practitioners (Taylor, 2017) (Bayne, 2012). 
As mentioned, the application is a Wear OS game that requires combining the use of both an Android phone and an Android watch to optimize its recognition accuracy and gamified experience. For activity recognition, our application used watch sensors for better accuracy. However, in many situations, participants may choose not to wear the watch. In those cases, when the watch was not connected, we used the built-in phone sensors instead, so that the game can run individually on the phone without the watch. The phone clearly offers more screen space and abilities such as typing messages.

\subsubsection{User Study Design}

\subsubsection{Participants and Group}

Forty-eight participants were recruited from both the local Ottawa area by posters and online through an Android Wear forum. Forty were recruited as main participants and eight as backups. The backup participants started and participated in the experiments as the main participants. Out of the 40 participants, 23 were males and 17 were females. Their average age was 26.93 years with a standard deviation of 6.07 years. We randomly divided our participants into four groups based on the versions of the app they received: Full (gamified and personalized), Gamified only, Personalized only, and the Control (neither personalization nor gamified, as a control group). The participants were randomly allocated to groups and well distributed with respect to exercise/player type, and physical activity level.

Note that the recommendations for the control group and the gamified group were created based on established exercise guidelines and were reasonable recommendations for the general population. Our choice for non-personalized groups follows closely the one-size- 
fits-all recommendations methods from the literature. Furthermore, the whole point of our study was to show the effectiveness of personalized recommendations. While we tried to offer a reasonable experience for non-personalized groups, the effectiveness of personalization, especially in terms of recommendation, was our hypothesis when other variables are held constant. Therefore, we did not use existing commercial applications for comparison in this study because we tried to avoid bringing in extra variables and possible confounding factors such as aesthetic and gameplay features that were not our focus.
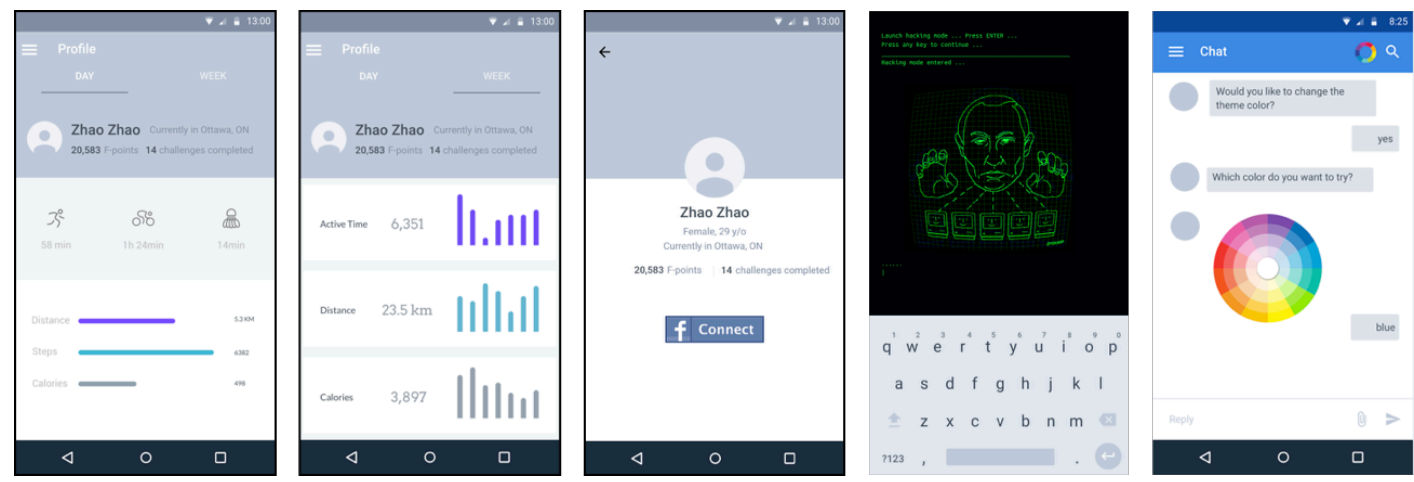

Figure 5.3: Example of game elements. From left to right: profile in daily view (including points and challenges), profile in weekly view (including points and challenges), connect to Facebook view, hacker mode view, theme color customization view
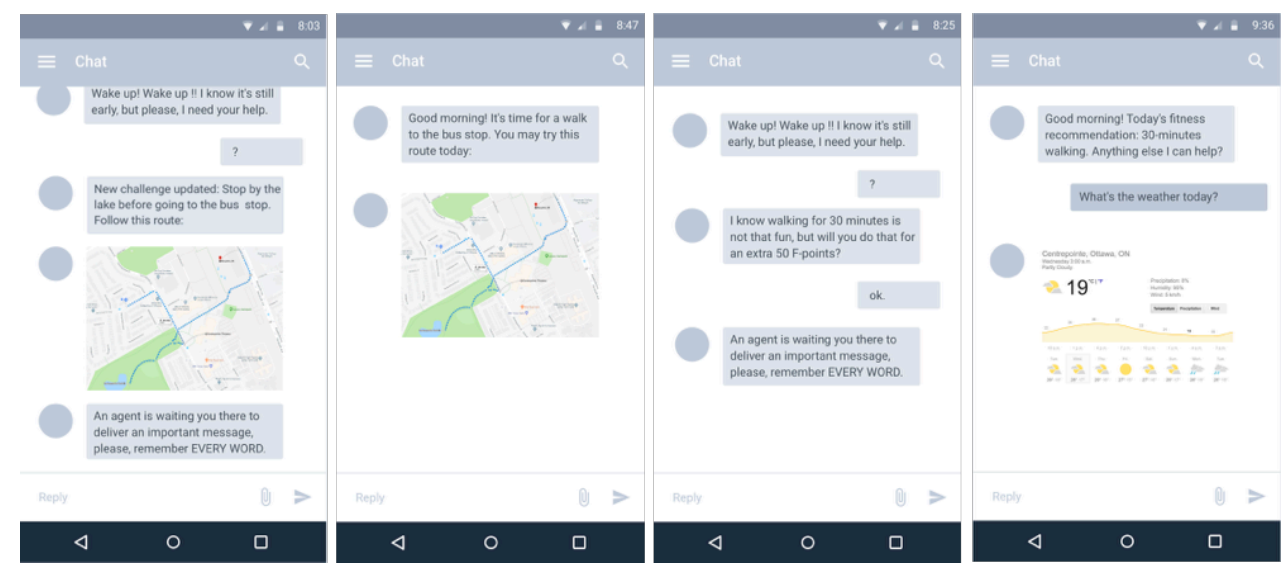

Figure 5.4: Example of recommendations for different groups. From left to right: Full, Personalized, Gamified, Control 
Figure 5.4 above shows an example of how recommending the same 30-minutes walking activity will look for the four study groups. The Full group received the recommendation through a gamified story (guided by the future self) with the game element of Challenge based on his/her player type of Achiever, and a personalized walking path. The Personalized group also received a personalized route without any game story or elements. The Gamified group received no personalized route but has the game story and the game element of Points (randomly assigned, because no player model is utilized for the Gamified group). The Control group received no personalization or gamification as a control group. In the screenshot of the Control group, we showed an example of how the weather assistant worked. Note that the example conversations were from screenshots and some context was not fully displayed.

To control the gamification level between groups, for the Gamified group, since there is no player model utilized, we randomly assigned a game element from Table 5.2 to each participant to make them at the same gamification level as the Full group.

We also limited the number of personalized recommendations to twice a day to eliminate the variability caused by frequent recommendations. The Gamified and the Control group (without personalization) received two messages per day at 9 am and $5 \mathrm{pm}$. We chose these two-time points because 9am is the time most of our participants were active. We did not send the notification earlier because we do not want to interrupt their sleep. We chose $5 \mathrm{pm}$ because most people close work at 5pm. The Full and the Personalized group received messages based on their individual player model. 
We did not set an ending date for this long-term study, instead, we set every 60 days as a study phase. At this time, we are at the end of phase 1, so the results presented in this paper are based on the date from the first 60 days. During the first 60 days, two of the 40 participants withdrew for different reasons, and two participants from the back-ups of the same study group were randomly selected to replace them.

\subsubsection{Procedure and Data Collection}

We asked participants to complete a pre-study questionnaire before providing them with the application. The questionnaire asked demographic questions including age, gender, height, weight, the number of hours they spend per week exercising, type of Android Wear owned, and the types and duration of video games (PC, console, and mobile) play. Two online questionnaires were provided and participants completed them, which provided us with the results to determine their player and exerciser type (Hexad, 2018) (8Colors, 2018). The application was distributed to participants through HockeyApp (Hockeyapp, 2018) after receiving participants' gamer and exerciser type results. Application features were selected based on the participant's player model.

For in-game data collection, we used Google Analytics API (GoogleAnalytics, 2018) to track participants' comprehensive in-app behavior data, including screen views and tapped events with an associated timestamp. We used Google Fit API to track user daily activity data, and a pop-up question asking participants if the recommendation they received that day was useful, this was pushed to participants every night at $9 \mathrm{pm}$

A post-study questionnaire was conducted at the end of the first phase of the study to comprehensively evaluate participants' experience during the first 60 days. First, we 
provided three general close-ended statements to measure participants' overall motivation, satisfaction, and preference with the in-game experience. Participants provided their responses on a 7-point Likert scale (from $1=$ strongly disagree to $7=$ strongly agree).

The statements were:

1) I find this kind of application motivating to exercise;

2) I was overall satisfied with this application;

3) I prefer using this type of application for exercise over regular exercise.

We adapted the Intrinsic Motivation Inventory (IMI) (IMI, 2016) measurement to assess participants' level of intrinsic motivation related to the game experience. Furthermore, we adapted the EMIC (European Microsoft Innovation Center) recommender system evaluation measurement (Knijnenburg, 2012) to evaluate the quality of our recommended activities. We also included Open-ended questions to obtain participants' comments and suggestions to improve the system. Participants received a $\$ 10$ honorarium gift card each to thank them for their participation in the study by the end of the first phase. Ethics approval was received from the related Ethics committee.

\subsubsection{Result and Analysis}

\subsubsection{General Information}

Out of the 40 participants, 23 were males and 17 were females. Their average age was 26.93 years with a standard deviation of 6.07 years. Their self-reported average hours of exercise per week prior to the study was 4.16 hours with a standard deviation of 2.96 
hours, while the average hours per week spent on playing video games (including pc, console, and mobile games) was 5.44 hours with a standard deviation of 4.13 . The selfreported average active hours raised to 4.58 after the study.

Participants were able to interact with the application through their Android phones or watches. Data shows that participants read $53 \%$ of messages on their phones and $47 \%$ on their watches. They tapped $36 \%$ of prompted choices on their phones and $64 \%$ on their watches. Results suggested that smartwatches were not only effective and more accurate for tracking activity data, but also feasible for some simple interactions such as reading messages and Tapping a choice from prompts. Users tended to interact with watches independently when completing simple tasks and switched to phones when different interactions were necessary (typing messages, etc.)

\subsubsection{Case Studies}

Below two case studies are presented as examples to show how our system recommends activities to different users in a typical week. Note that if there is any more information or any change the system found during the week, the recommendations adjust accordingly.

\section{Case Study A}

Participant A was female, 26y/o, student, 5'8', 135lb, 20.5 BMI (normal weight), no serious health issues, and currently taking no medications. Player type: Free spirit, Fitness color: White. The proposed system detected Participant A takes the bus to university every Monday, Tuesday and Thursday, and mostly stays at home for the rest of the week. She attends a group cycling class once a week on Friday evening for half-hour. 
According to GRPAH, Adults aged 18-64 were encouraged to do 300 minutes of moderate-intensity aerobic physical activity throughout the week for good health benefits (He, 2014). People with Exerciser type of White prefer hiking, running, yoga, cardio and gym strength training. When accessing her calendar the system found she had two dinner reservations on Thursday and Saturday night both at $6 \mathrm{pm}$ for the coming week. The proposed system generated the following recommendations:

Extend the walking distance to the bus stop on every school day (both morning and afternoon, overall 45 minutes of walk per each school day); 30 minutes' walk for nonschool days after dinner; A one-hour home Yoga session on Tuesday $7 \mathrm{pm}$ when the user is generally not active; A hiking morning on Saturday in a nearby park. The player type of Free spirit was assigned the game feature of 'theme color customization'. So, the reward of completing recommended activities for Participant A was to unlock different theme colors.

\section{Case Study B}

Participant B was male, 35y/o, software developer, 5'11', 1651b, 23.0 BMI (normal weight), no serious health issues, and currently taking an over-the-counter pain reliever for his back pain. Player type: Achiever, Fitness color: Red. Our system detected Participant B drives to work every Monday, Tuesday, Thursday, and Friday (15 minutes' drive). On Wednesday, he works from home. He plays basketball every Wednesday night from $8 \mathrm{pm}$ to $9 \mathrm{pm}$ and every Saturday morning from 9am to $11 \mathrm{am}$. Exerciser type of Reds like Basketball, tennis, racquetball, in-line skating, Frisbee, mountain biking, soccer, 
skiing. The proposed system found Participant B was almost as active as GRPAH recommended, but the type of activities he did was limited to basketball.

The system recommended a one-hour tennis/racquetball session on Wednesday night instead of basketball, daily 15 minutes' walk after work and a 60-minutes' walk (in a nearby park) on Sunday morning. The player type of Achiever was assigned the game feature of challenge. So, the system provided recommendations to Player B in the challenge style.

\subsubsection{Overall Motivation and Satisfaction}

A one-way between-groups analysis of variances (ANOVA) and post-hoc Tukey-Kramer HSD test (Jaccard, 1984) was conducted to analyze the main effects between four groups. The alpha was set at 0.05 for all statistical tests.

Figure 6.4 shows the average and standard deviations of the scores for the first three general questions assessing participant motivation, satisfaction, and game preference. An asterisk indicates there were significant results found between groups.

The results show there were statistically significant differences between groups was determined by one-way ANOVA for overall motivation $(\mathrm{F}(3,36)=22.49, \mathrm{p}<0.0001)$, satisfaction $(F(3,36)=22.12, p<0.0001)$, and preference $(F(3,36)=15.0, p<0.0001)$.

Post-hoc comparisons using the Tukey HSD test indicated that for all three questions, the mean score for the Full, Personalized, and Gamified group was significantly different from the Control group, respectively. This means that in general, gamification and personalization both have a positive effect on user motivation, satisfaction, and 
preference, as seen between-group (Full, Control) (Personalized, Control) and (Gamified, Control). Moreover, for motivation, the mean score for the Full group was significantly different from the Personalized group. The pairwise comparison significance is also marked in Figure 6.4. This significance implies that Gamification can also add more motivation to a personalized fitness recommendation system, as seen between the Full group and the Personalized group in motivation.

\subsubsection{IMI Sub-scales}

The IMI scale was adapted to the current game context. Figure 5.5 shows the average and standard deviations of the scores for each IMI sub-scale questions. Results indicate that there were statistically significant differences between groups as determined by a oneway ANOVA for interest/enjoyment $(F(3,36)=24.24, p<0.0001)$, perceived competence $(\mathrm{F}(3,36)=4.60, \mathrm{p}<0.01)$, effort/importance $(\mathrm{F}(3,36)=8.01, \mathrm{p}=0.001)$, and value/usefulness $(F(3,36)=15.90, p<0.0001)$.

The Tukey-Kramer HSD test results indicated that for interest/enjoyment, the mean score for the Full, Personalized, and Gamified group was significantly different from the Control group. Moreover, the pairwise comparison result shows the mean score for the Full group was significantly different from the Personalized group. For perceived competence, significant differences were found between the Full group and the Personalized group, and between the Full group and the Control group. For Effort/importance, significant differences were found between the Full group and the Gamified group, the Full group and the Control group, the Personalized group and the Gamified group, as well as between the Personalized group and the Control group. For 
Value/usefulness, significant differences were also found between the Full group and the Gamified group, the Full group and the Control group, the Personalized group, and the Gamified group, as well as between the Personalized group and the Control group. The pairwise comparison significance is also marked in Figure 5.5.

The IMI results indicate that gamifying increases players' interest in and enjoyment of the personalized recommendation system, (significant between the Full group and the Personalized group in interest/enjoyment). Personalization contributes more towards promoting effort/importance as well as value/usefulness compared to gamification (significant between the Personalized group and the Gamified group).

\subsubsection{EMIC Recommender System Evaluation}

Figure 5.6 shows the average and standard deviations of the scores for each EMIC subscale questions. The results show there were statistically significant differences between groups, determined by one-way ANOVA for perceived recommendation quality $(F(3,36)=108.77, p<0.0001)$, perceived system effectiveness $(F(3,36)=26.52, p<0.0001)$, and system-specific privacy concern $(\mathrm{F}(3,36)=58.37, \mathrm{p}<0.0001)$.

The Tukey-Kramer HSD test results indicated that for both perceived recommendation quality and perceived system effectiveness, the mean scores for Full and Personalized groups were significantly different than Gamified and Control groups that were not personalized. For system-specific privacy concerns, the mean scores for the Full group and the Personalized group were also significantly different than the Gamified group and the Control group because for non-personalized groups we did not ask to access users' personal data. Moreover, a significant difference was also found between the Full group 
and the Personalized group. The pairwise comparison significance is also marked in Figure 5.6.

The results suggested that our system is effective at providing daily fitness recommendations to participants (comparing the Full group and the Gamified group, and the Personalized group and the Control group) with respect to both perceived recommendation quality and perceived system effectiveness. We also found that, as expected, participants were concerned about privacy when the system has a player model and asks for more permissions to access their personal data (comparing the Full/Personalized and Gamified/Control groups). On the other hand, gamification reduced some of the concerns (significant difference found between the Full and the Personalized groups). Privacy concerns are important yet beyond the scope of this work. Still, we believe the noticed effect of gamification can be of value in future research and design.
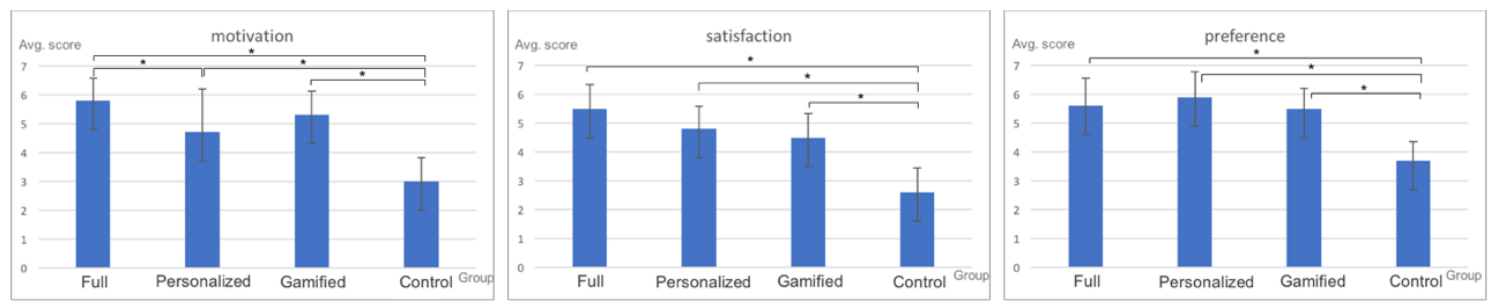

Figure 5.5: Results for post-study questions 1, 2 and 3. From left to right: overall motivation, overall satisfaction, and overall preference over regular exercise 


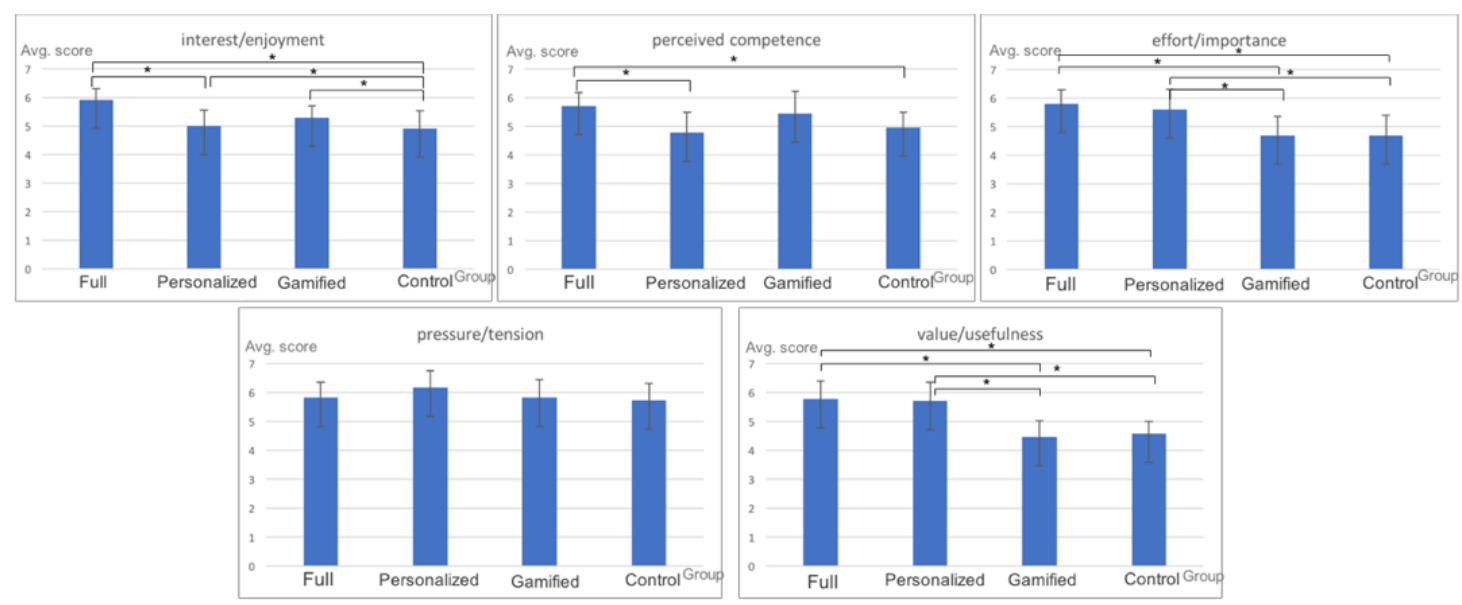

Figure 5.6: Average and Standard deviation of IMI: Interest/Enjoyment, Perceived Competence, Effort/Importance, Pressure/Tension and Value/Usefulness.

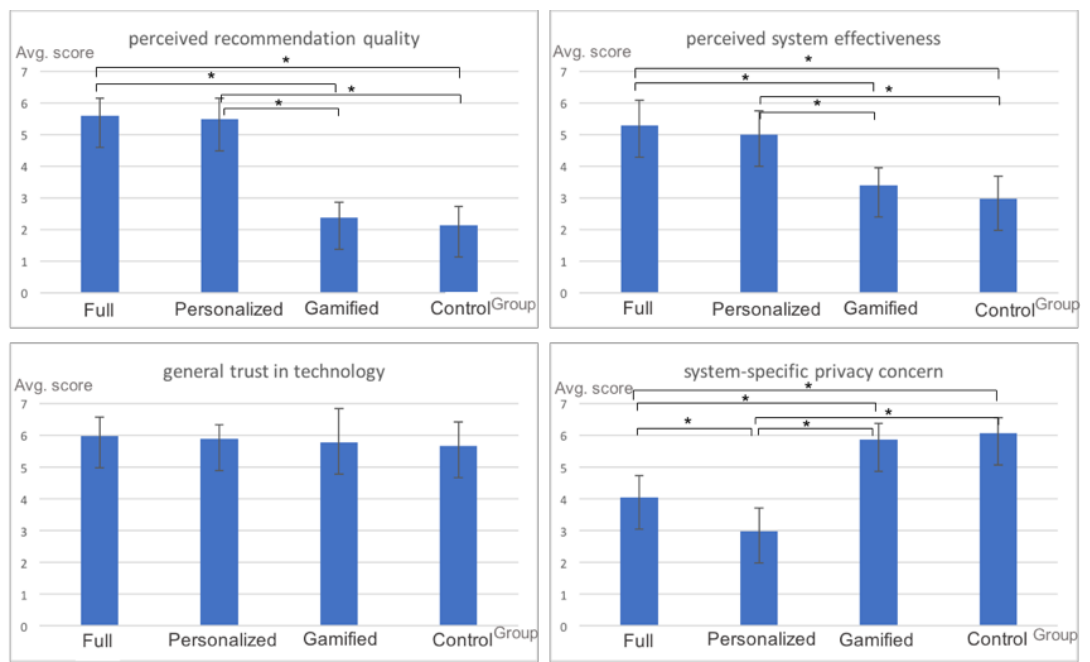

Figure 5.7: Average and Standard deviation of EMIC: perceived recommendation quality, perceived system effectiveness, general trust in technology, system-specific privacy concern.

\subsubsection{Daily Statistical Data}

As mentioned before, Google Analytics API was utilized to track users' comprehensive in-app behavior data, and Google Fit API was used to track user daily activity data including steps, calorie consumption, etc. Figure 5.8 below shows some daily statistical data: the change of active users of all four groups during the 60 days of study (top-left); the change of daily total number of conversation user send to the system (top-right); the 
daily average active calorie consumption excluding basal metabolism (bottom-left); and the daily change of the number of useful recommendations (bottom-right).

From Figure 5.8 we can see for daily active users (a) and daily conversations (b), there is an overall descent in trends appear as time increases for all four groups. Among them, the Full group maintains a relatively higher value compared to the other three groups, and participants in the Full, Personalized and Gamified group interacted with the system more than the Control group, see Figure 5.8b. With respect to the daily active use and daily conversations Figures 5.8a and b, when comparing the Personalized group and the Gamified group, we can see the value of the Gamified group were higher than the Personalized group in the early phase of the study but were surpassed by the Personalized group in the late phase of the experiment (around 35 to 40 days). These results indicate that both personalization and gamification could have a positive impact in promoting user engagement with the system, however, while gamification could bring more interactions in short term (within a month), personalization could lead to a more sustained engagement (over a longer time). 


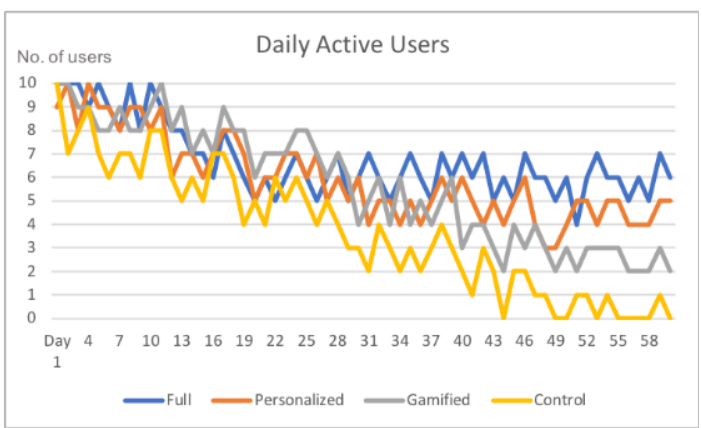

(a) Daily active users

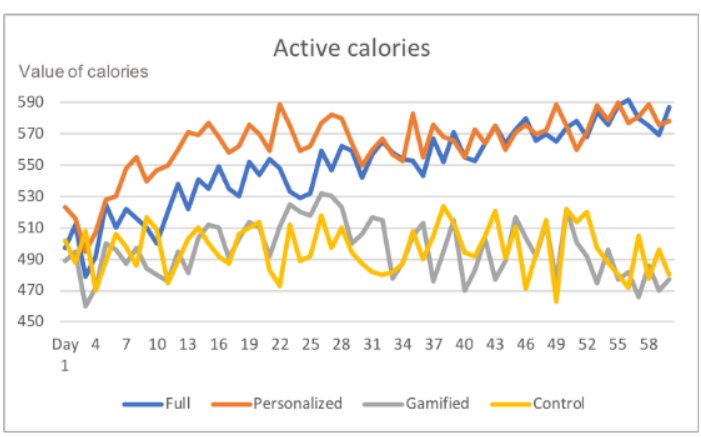

(c) Daily average active calories

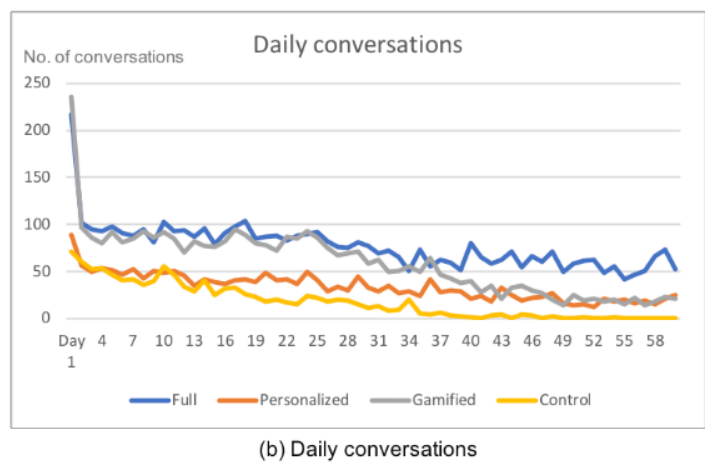

(b) Daily conversations

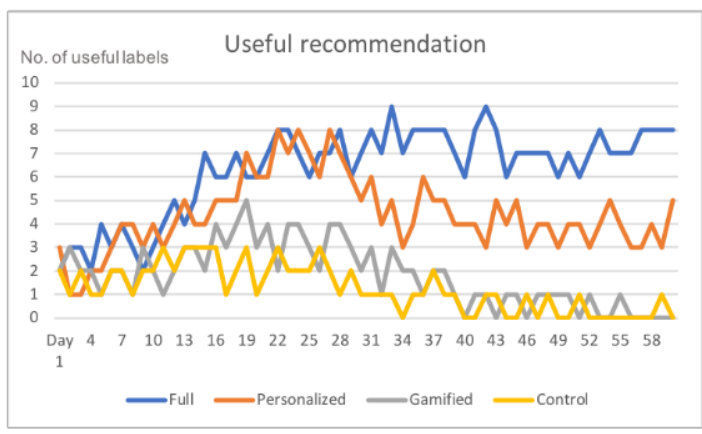

(d) Daily useful recommendation

Figure 5.8: Daily Statistical Data

For active calories, Figure 5.8c, we can see slight ascent trends for both the Full group and the Personalized group, and flat trends for the Gamified group and the Control group. The Full group started with a lower average calorie consumption compared to the Personalized group but shows in almost equal value in the end (higher growth rate). These results indicate that personalization could have a positive impact on promoting actual physical activity, which exclusive gamification could not. However, adding gamification elements to a personalized fitness recommendation system could result in a higher increase rate in daily physical activity. For useful recommendations Figure 5.8d, the value of the Full group increased in the first half of the study and then kept flat, the Personalized group also increased first, followed by a decrease, then kept flat. Note that 
the active calorie measures the calorie burnt during fitness activities. Basal metabolic was excluded.

The increase in the Full group and the Personalized group can be attributed to the continuously updating player model that will improve recommendations over time. The Gamified group and the Control group (without player model) showed descending trends approaching zero. The results suggest that our system is able to generate useful fitness recommendations by using the player model, and users considered the recommendation more useful when gamification elements were added.

\subsubsection{Qualitative Results}

Open-ended questions were asked at the end of the post-study questionnaire. We received many comments/suggestions on how to improve our system, which mainly focused on five aspects, as shown in Figure 5.9. As shown the customized storyline was the most requested feature, followed by multiplayer mode, more quality recommendation, the feature for setting and tracking fitness goals, as well as adding more location-based features. This feedbacks laid the foundation for planning our future work in this research.

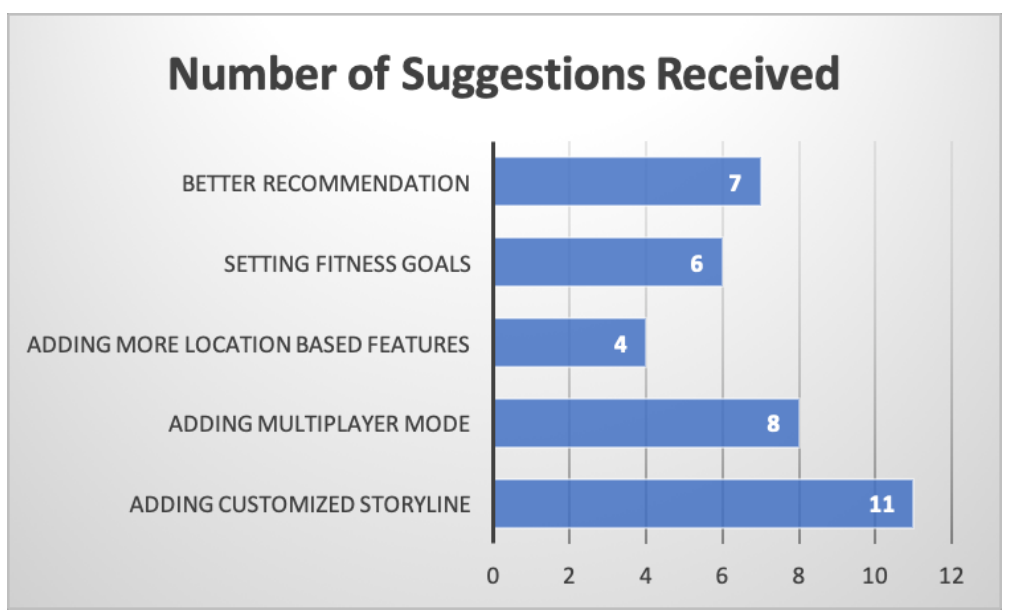

Figure 5.9: Number of Main Suggestions Received from Open-ended Questions 


\subsubsection{Discussion}

Overall in this 60-day user study, the hypotheses were verified that 1) it is feasible to generate personalized exercise recommendations with player modeling, and 2) the combination of player modeling and gamification could enhance user engagement with the system, as well as promote actual physical activity.

More specifically, gamification was found to promote engagement but in the short term, as can be seen, the gamification group has better results early on. As the experiment moved on, the trend changed, and the personalized group improved. This can be attributed to the player modeling that requires time to reach a minimum level of precision in reflecting the player's characteristics before it could offer reasonable recommendations. After that, the player modeling helped sustain the activity level in the long term. This suggests that activity recommendations based on player modeling can be an effective and promising approach of creating personalized fitness experiences over longer periods while gamification can help attract the users and create the initial interest.

Our findings confirm previous work suggesting the value of gamification and player modeling, extend them to the area of fitness assistants, and provide new insights on the value of continuous activity tracking and player modeling as opposed to the more common static and one-time modeling. The presented results have direct implications for the design of fitness assistants and potentially other recommender systems. 
Gamification is good, but not enough!

Previous work by authors and other researchers have shown the potential value of gamification to increase engagement, but they also have highlighted the issue of retention. Players tend to leave the game once it is well experienced. While adding new features can be a reasonable way of keeping users engaged, it is hard and costly to implement due to constant design and upgrade. The ability to understand users and their dynamic life and provide gameplay features that match the users' activities can be a way to introduce change and novelty while keeping the development cost under control.

\section{Player Modeling: Personalization vs Categorization}

The idea of categorizing users to provide them with customized service is appealing but ignores the individual differences which are often significant. The availability of personal data, as a result of various methods of collecting information, suggests that the users can be understood as individuals and not members of a category. This true personalization allows a new level of customization that will potentially offer users a much more appealing and effective experience. Our results show the potential relevance of this idea to the field of fitness assistants. The more we understand the user, the more personalized our recommendations will be which will, in turn, result in more effective recommendations. Developing a comprehensive model that involves various user characteristics (from personality type to daily routines) can help understand the user properly. 


\section{Adaptive and Continuous Modeling}

While many games and other applications rely on a certain user model, in most cases this is done as a one-time static decision assigning the user to a certain group. Our study shows the value of not only having a more comprehensive personal model but also allowing it to evolve and adapt using on-going data from the user. This constantly tunes the model and makes the recommendations more effective. Using such adaptive and dynamic models can enhance the performance of such applications and we recommend the designers to consider it when possible.

\section{4/7 Recommendation}

Fitness and health are not limited to fitness centeres. Being active is a lifestyle and so the activity recommendations should not be limited to a particular time. In the absence of a dedicated personal trainer, an intelligent fitness assistant, equipped with a detailed player model, can offer $24 / 7$ recommendations for being active that take various user's contexts into account. Our results show the potential value of this approach which can be improved with more comprehensive personal data and a better database of activities and gameplay features. While our system provided all-day and continuous modeling and recommending, it is worth noting that the users did not wear the activity trackers during sleep and we did not track any sleeping activities. As such, while the system was able to perform non-stop, in practice it was paused during sleep times (night or day). 


\section{Limitations}

There were certain limitations in the proposed system and the performed study, some mentioned by the participants, that we believe were not critical enough to significantly affect the findings but are still worth noting and improving in the future work.

This study relied on a simple game that we designed ourselves with a simple story/gameplay. This may have negatively affected the players' attraction and engagement. The game could be designed through a more rigorous process, or we could somehow allow customization and choice or potentially use another existing game. There was also no multiplayer option which is ignoring the social aspects of gaming and active lifestyle and again could negatively affect user engagement. When designing different gamified features for different types of players, we assigned only one game element to each type of player. This may not be adequate for targeting individual users.

The 8-color-of-fitness system was used as a model to suggest activities. This was due to the fact that the research group did not find any other alternatives and needed to rely on a fairly acceptable method. This system is by no means ideal and has its own limitations. It can be replaced with any other method such as other models, an interactive trainer, or a trained expert system.

Android Activity Recognition API was used for activity tracking and predicting in this work. It is only able to recognize 6 simple physical activities. For more complex daily activity, it required manual labeling from users within the conversation. This may bring complexity to participants. Also, only Android Wear users were considered and limited each group to 10 members which may not be adequate enough. 


\subsection{Personalized Physical Activity Recommendation with Machine Learning}

\subsubsection{Overview}

The results of previous studies show the feasibility and effectiveness of using player modelling for fitness activity recommendation. However, the parameters for building the player model were selected intuitively based on our limited knowledge of the problem domain. Therefore, parameter selection - the process of selecting a subset of relevant parameters for use in model construction - can be optimized in building our player model.

The player model we used in previous research consists of four sub-models (activity recognition model, general model, exerciser type model, and player type model), which covered parameters of user's age, gender, weight, height, recognized daily activities, steps, active calories, walking/running distances, calendar events, location, as well as user's player type (Hexad model) and exerciser type (8colors model). From the existing literature, there were some potential parameters that could be added to our system for investigation, including users' lifestyle information such as sleeping habits, type of occupation, measures of stress, etc., (Sami, 2008).

On the other hand, as mentioned in the previous section, the 8-color-of-fitness system was used as a model to suggest activities. Thus, the recommendations of activity types that our system generated were mostly based on personalities. This was due to the fact that the research group did not find any other alternatives and needed to rely on a fairly acceptable method. Therefore, the method of mapping player model to the right type of activities for recommendation can be further investigated. 
In summary, the recommendation system, especially for recommending different types of physical activities, could be further optimized. Thus, the remaining research questions left for this study (RQ5-7)were:

- What parameters should exist in the player model?

- Which existing models and approaches are effective to recommend specific activities to different users?

- How the model could be used to recommend activity?

\subsubsection{Survey}

In order to answer these questions above, firstly, a survey was distributed to gather data regarding people's exercise preferences (potential parameters were selected based on our literature review). In addition to regular demographic and health information like age, gender, weight, height, etc., lifestyle information, as well as other potential parameters were also collected from our participants, for example, diet constraints, sleeping patterns, activity tracker owned, etc. Our survey consists of 30 items of variables. A brief list of survey items is shown in Table 5.3.

The detail of the survey items is shown in Appendix D. The survey was conducted online from Mar 10, 2019, until Apr 30, 2019. An invitation to participate in the survey was sent by e-mail from our research group to our colleagues and friends, as well as our previous research participants. We have collected a total of 178 responses. 
Table 5.3: List of Survey Items

\begin{tabular}{|c|c|c|c|}
\hline Basic info & Lifestyle info & Objective info & Other parameters \\
\hline Age & Get-up time & $\begin{array}{c}\text { Exercise importance } \\
\text { level }\end{array}$ & No. of children \\
\hline Gender & Bedtime & Purpose of exercise & Pets owned \\
\hline Height & Nutritional supplement & Amount of exercise & Favorite exercise music \\
\hline Weight & Exercise frequency & $\begin{array}{c}\text { Enjoyment level of } \\
\text { different exercises }\end{array}$ & Cell phone system \\
\hline Occupation & Exercise partner & & $\begin{array}{c}\text { Wearable activity } \\
\text { trackers owned }\end{array}$ \\
\hline $\begin{array}{c}\text { Medical limitation for } \\
\text { exercise }\end{array}$ & Exercise trainers & & $\begin{array}{c}\text { Favorite type of soft } \\
\text { drink }\end{array}$ \\
\hline Residence & Gym mate & & $\begin{array}{c}\text { Favorite types of video } \\
\text { game }\end{array}$ \\
\hline & Vegetarian & & Favorite sport brand \\
\hline & Daily commute methods & & 8-colors of fitness type \\
\hline & Typical daily routine & & \\
\hline
\end{tabular}

For classification purposes, we preprocessed the data by labeling the user's preference for each type of exercise. For each exercise (in question 31), we define -1: negative experience (not at all enjoyed), 0: never tried (N/A), and 1: positive experience (including slightly enjoyed, very enjoyed and extremely enjoyed). We trained a binary prediction model (with only non-zero data and using SVM) for each individual Physical Activity (PA). We chose Support Vector Machine (SVM), a supervised learning algorithm, because it is effective in high dimensional spaces and especially works well on small datasets. There are many other classification algorithms that can be used in this prediction model for comparison and optimization. The choice of the learning and prediction method was not one of our research questions and as such we chose only one possible option to develop our prototype. Improving this part of the system is one of the future direction of the research. We split our data into $80 \%$ training data and $20 \%$ testing data. For those physical activities with testing accuracy $(\mathrm{TP}+\mathrm{TN}) /$ total sample size $>=70 \%$, 
we conducted permutation feature importance experiment to compute feature importance scores, as explained in the following paragraph. Because our dataset is relatively small so we chose the accuracy bigger than $70 \%$ to be an acceptable prediction. Note that directories of different types of sports and exercises (BBC, 2019) are used in this research as potential physical activities for the recommendation.

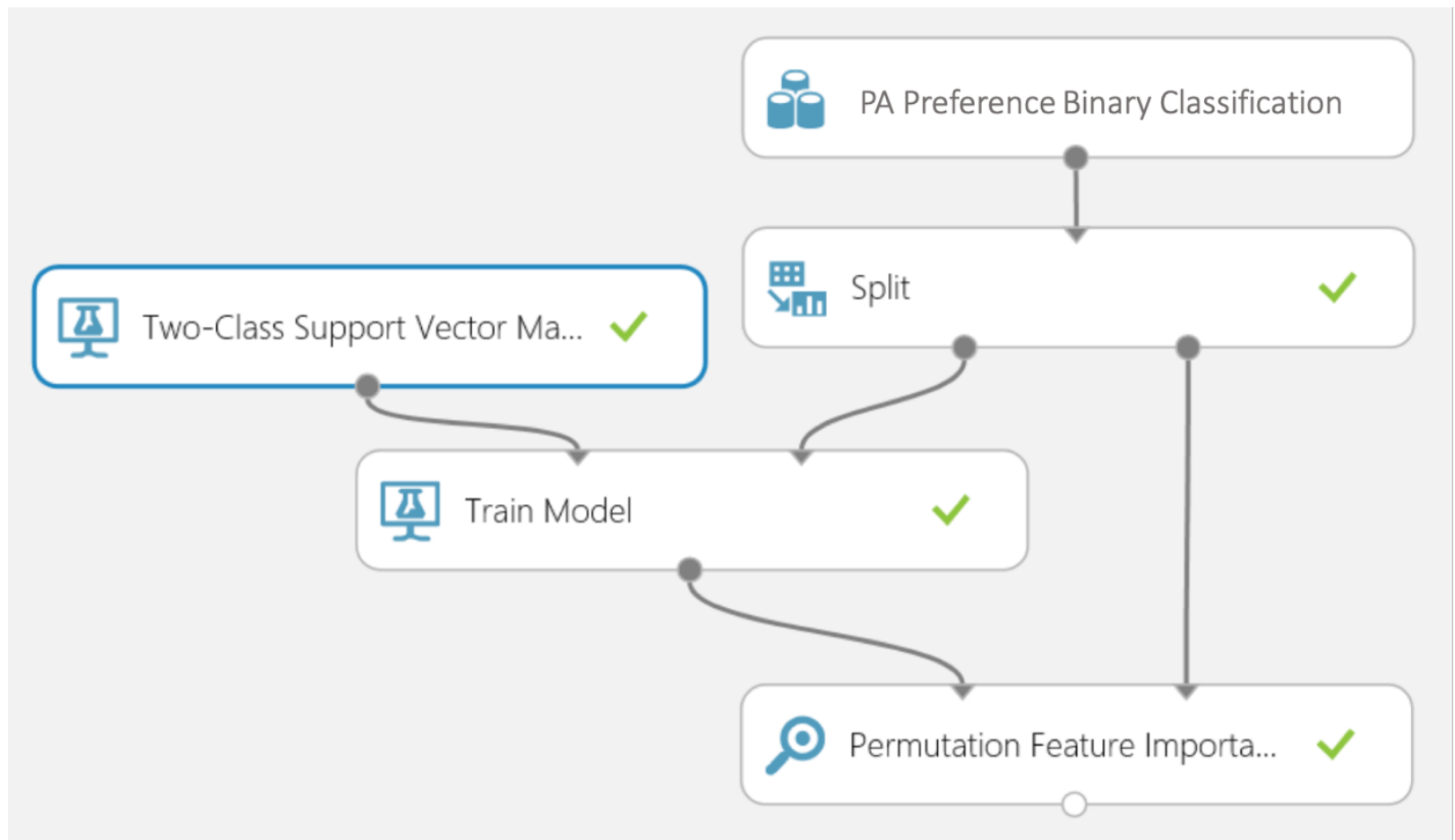

Figure 5.9: Overall Workflow of the Feature Importance Experiment in Azure

The experiment of evaluating which features were more important was done in Microsoft Azure Machine Learning Studio. For each individual Physical Activity, we conducted the following experiment:

- $\quad$ Add the PA Preference Binary Classification to the experiment.

- $\quad$ Add a Split module to create a training and test datasets.

- Add a Two-Class Support Vector Machine module to initialize the SVM classifier. 
- Add a Train Model module to train the classifier, and connect the SVM module to the left input port and the training dataset to the right input port.

- Add a Permutation Feature Importance module and connect the trained model and the test dataset to the left and right input ports respectively. Set the Metric for measuring performance property to Classification - Accuracy.

Then we conducted the experiment in Azure, click on the output port of Permutation Feature Importance, and select visualize to inspect the output results of the module. The following figure 5.10 shows an example of the list of features sorted in descending order of their permutation importance scores.

We conducted the experiment for each individual physical activity, then computed the average importance score for each feature. Note that for different physical activities, the corresponding rank of the important features varied obviously, but the overall goal of this experiment is to find the important features for building a player model in general, so we used the average score here. The top 10 features with relatively higher average feature importance score were selected as the features for the final classification model. 
Feature
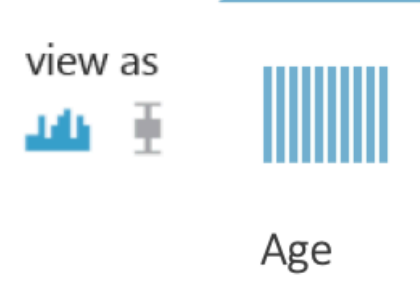

Gender

Partner

Video game

Getup time

Occupation

Residence

Wearable Trackers

Importance

Children

Trainer

Pets
Score

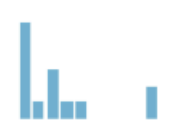

0.038462

0.034837

0.029738

0.027364

0.021753

0.018464

0.013746

0.009364

0.008263

0.006234

0.003347

0.001832

Figure 5.10: Example of Feature Permutation Importance Scores (Basketball)

\subsubsection{Application Redesign and Implementation}

\subsubsection{Recommendation System Design}

The overall recommendation process of the system is outlined in Figure 5.11. 
For those PA with accuracy $>=70 \%$, we directly use the prediction model to decide whether to recommend a certain type of physical activity to a new user or not (see the left branch).

For other types of PA, we employed a collaborative filtering method with K-means clustering (Wagstaff, 2001). Unsupervised cluster analysis is performed using the survey data (for the only PA with accuracy $<70 \%$ ) (see the right branch). The $\mathrm{K}$ value of 4 was generated with elbow method (to define clusters such that the total within-cluster sum of square (WSS) is minimized. (Kodinariya, 2013)) The K value was calculated using fviz_nbclust() function in factoextra R package (Kassambara, 2017). For each cluster, we found their top-5 (out of 13) popular PA.

We aimed to recommend 10 PA to each individual user. Set R in figure 5.11 represents the result of the final 10 PA for recommendation, Set A represented those PA with positive results from the binary classification model, Set B represented the top five popular PA of the cluster which the target user belongs to, and Set $\mathrm{C}$ included five randomly selected PA (excluded those PA in Set B), as an alternate set in case the number of item in Set A is less than five. At this stage, the PA in Set R was recommended to our users in a randomized order, because we did not consider the weight of different PA in the prediction model. 


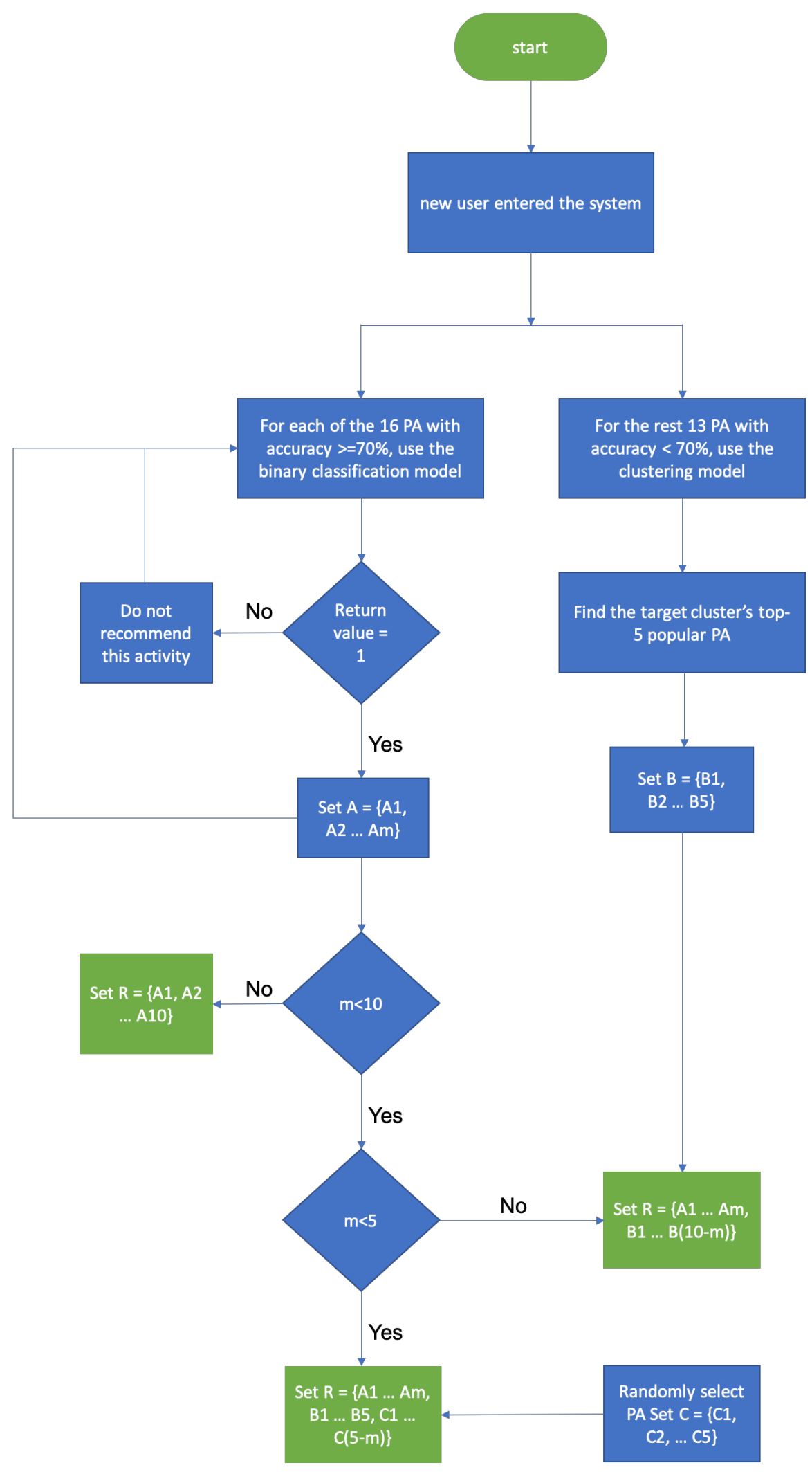

Figure 5.11: Overall Recommendation Flowchart 


\subsubsection{Model Updates}

Furthermore, the PA prediction model in this proposed work kept updating itself. Thus, when any new user data entered the system, it will be added to the dataset to re-train the prediction model. For instance, if a PA was recommended to a user, but the user provided negative feedback to the recommendation afterward, the system used this feedback to label the corresponding PA, and the prediction model was re-trained to include this data. Ideally, as more users entered the system, the accuracy of the prediction model, and the quality of the clustering improved. In order to achieve this, we have added a feedback system into the original application. Users could provide feedback on any of the recommendations generated by the system, by either tap "I liked it" or "I didn't like it" buttons, or leave any specific comments in the comment box.

\subsubsection{Result and Analysis}

Overall, we have collected 178 survey responses. Out of the 178 participants, 95 were males and 83 were females, with $17.4 \%$ age between 18 to $24,34.8 \%$ age between 25 to $34,26.4 \%$ age between 35 to $44,12.9 \%$ age between 45 to $54,6.2 \%$ age between 55 to 64 , and $2.2 \%$ age between 65 to 74 . This study covered 29 different types of physical activities (PA). Our player model consisted of 30 features (29 questions from the survey and an additional BMI calculated from height and weight).

\subsubsection{Feature Importance}

There were 16 out of 29 types of PA resulted in a prediction accuracy $>=70 \%$, the remaining 13 types of exercises resulted in relatively lower accuracy, as summarized in 
Table 5.4 below. As an example, if we look at the PA basketball, in our 178 survey responses, 121 of them have either positive experience (1) or negative experience $(-1)$ for basketball. The rest 57 replied N/A (0) because they never actually tried it. So, as we can see in table 5.4 , the response rate for basketball is $121 / 178=68 \%$. For those 121 valid data, we use $80 \%$ of it (97) for training the model and the rest $20 \%$ (24) for testing the model (as described in section 5.2.2). We compared the predictive result with the actual label ( 1 or -1$)$, then calculate the accuracy. (For the 24 testing data, we predict 20 of them correctly and 4 of them wrong, so the accuracy is $20 / 24=83 \%$ ).

Table 5.4: Accuracy for the Binary Classification Prediction Model

\begin{tabular}{|c|c|c|c|}
\hline & Type of exercise & $\begin{array}{c}\text { Response rate (non- } \\
0 \text { value) }\end{array}$ & Accuracy \\
\hline \multirow[t]{16}{*}{ Accuracy $>=70 \%$} & Walking or jogging & $100 \%$ & $81 \%$ \\
\hline & Running & $100 \%$ & $74 \%$ \\
\hline & Hiking & $97 \%$ & $80 \%$ \\
\hline & Swimming & $85 \%$ & $73 \%$ \\
\hline & Riding a bike & $86 \%$ & $76 \%$ \\
\hline & Dancing & $74 \%$ & $85 \%$ \\
\hline & Skiing & $58 \%$ & $72 \%$ \\
\hline & Ice-skating & $69 \%$ & $79 \%$ \\
\hline & Golf & $32 \%$ & $81 \%$ \\
\hline & Soccer & $41 \%$ & $78 \%$ \\
\hline & Hockey & $13 \%$ & $74 \%$ \\
\hline & Basketball & $68 \%$ & $83 \%$ \\
\hline & Horse-riding & $21 \%$ & $73 \%$ \\
\hline & Snooker or billiards & $89 \%$ & $78 \%$ \\
\hline & Yoga & $42 \%$ & $81 \%$ \\
\hline & Conditioning exercise & $37 \%$ & $71 \%$ \\
\hline \multirow[t]{6}{*}{ Accuracy $<70 \%$} & Aerobics & $94 \%$ & $54 \%$ \\
\hline & Weight lifting & $33 \%$ & $66 \%$ \\
\hline & Pilates & $8 \%$ & $62 \%$ \\
\hline & Roller skating & $72 \%$ & $68 \%$ \\
\hline & Extreme sports & $9 \%$ & $48 \%$ \\
\hline & Martial arts. Boxing or wrestling & $4 \%$ & $46 \%$ \\
\hline
\end{tabular}




\begin{tabular}{|c|c|c|c|}
\hline & Tennis or badminton & $86 \%$ & $64 \%$ \\
\cline { 2 - 4 } & Bowling & $63 \%$ & $64 \%$ \\
\cline { 2 - 4 } & Football & $20 \%$ & $57 \%$ \\
\hline Rugby & $37 \%$ & $55 \%$ \\
\hline Volleyball & $56 \%$ & $62 \%$ \\
\cline { 2 - 4 } & Fishing & $43 \%$ & $68 \%$ \\
\cline { 2 - 4 } & Sailing, wind-surfing or boating & $16 \%$ & $55 \%$ \\
\hline
\end{tabular}

The results of the average feature importance score were summarized in Table 5.5 below.

Figure 5.12 visualized the result in descending order. As we can see from the result, the most important parameters (top 10) in this prediction model were the user's age, purpose of exercise, if vegetarian or not, get up time, video game preference, if workout with a trainer or not, gender, BMI, favorite type of music when exercising, and favorite sport brand.

Table 5.5: Average feature importance score for all model parameters

\begin{tabular}{|c|c|}
\hline Feature & Average feature importance score \\
\hline Age & 0.033984 \\
\hline Gender & 0.027345 \\
\hline Weight & 0.003762 \\
\hline Height & 0.002397 \\
\hline BMI & 0.026455 \\
\hline Occupation & 0.015428 \\
\hline 8 Colors type & 0.006964 \\
\hline Get-up time & 0.029765 \\
\hline Bedtime & 0.017492 \\
\hline Nutritional supplement & 0.003148 \\
\hline Exercise importance & 0.004654 \\
\hline Exercise frequency & 0.005054 \\
\hline Why exercise & 0.032987 \\
\hline Exercise partner & 0.011794 \\
\hline Medical limitation & 0.000349 \\
\hline Amount of exercise & 0.000130 \\
\hline Trainer & 0.027671 \\
\hline
\end{tabular}




\begin{tabular}{|c|c|}
\hline Residence & 0.016504 \\
\hline Vegetarian & 0.030217 \\
\hline Children & 0.013856 \\
\hline Pets & 0.010479 \\
\hline Exercise music & 0.023982 \\
\hline Type of Phone & 0.008344 \\
\hline Wearable trackers & 0.013067 \\
\hline Soft drink & 0.000228 \\
\hline Video game & 0.028630 \\
\hline Gym mate & 0.013893 \\
\hline Sport brand & 0.018764 \\
\hline Transportation & 0.002905 \\
\hline Typical daily routine & 0.000031 \\
\hline
\end{tabular}

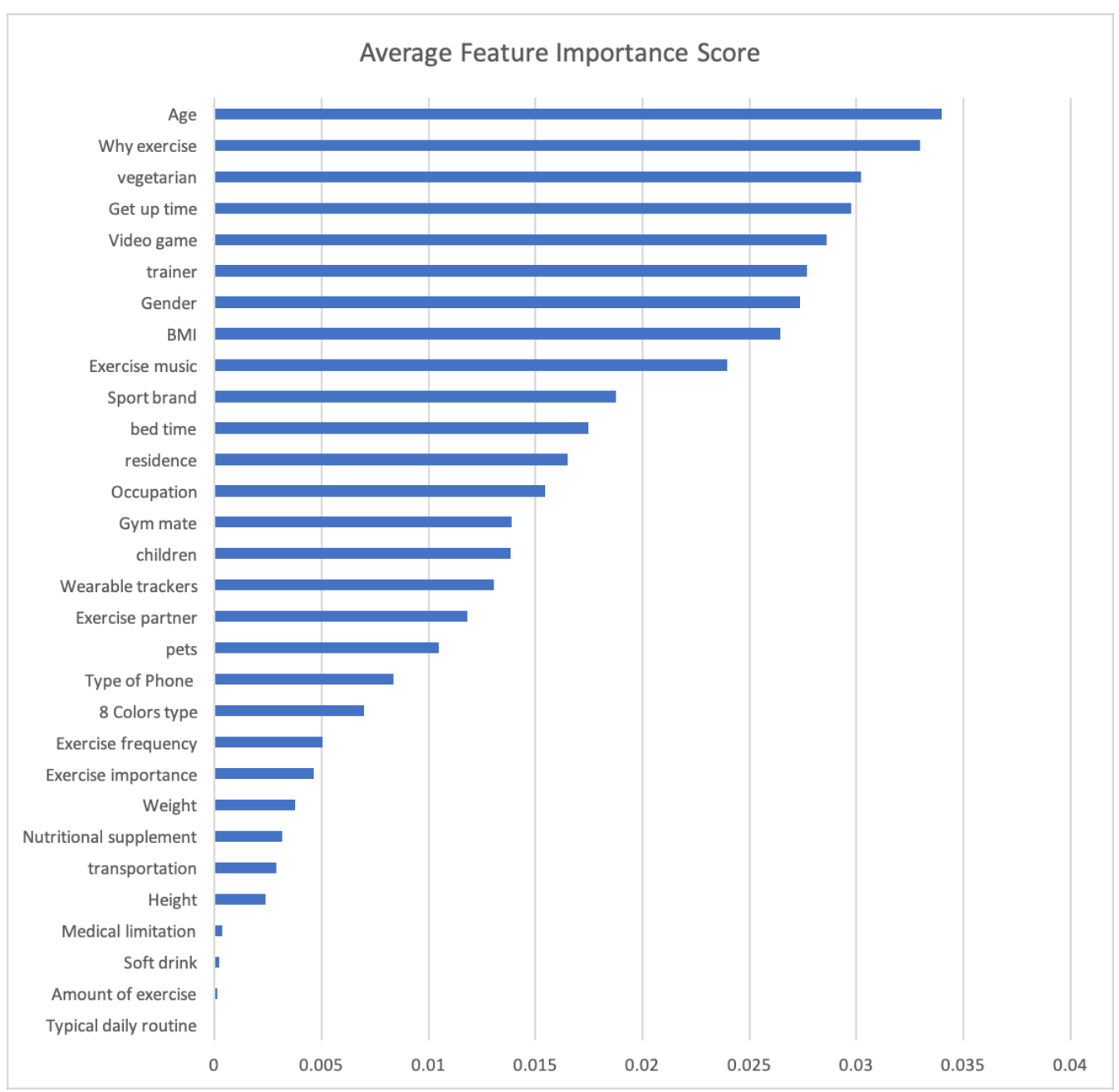

Figure 5.12: Average feature importance score for all model parameters (in in descending order) 


\subsubsection{Comparison with The 8-Color Model}

For those 29 different types of physical activities covered in this study, we did not find any significant correlations between the 8-colors of fitness results and our participants' physical activity preference. Thus, we believe that personality type has only a limited influence on people's preference toward physical activities. On the other hand, other parameters such as an individual's lifestyle, physical conditions, and social connections, may play more important roles in determining their exercise taste. Table 5.6 below shows an example (result of one of our participant UserID \#97) comparison between our prediction model and the 8-colors model.

Table 5.6: Example Comparison of Predictive Results between the 8 Color Model and our Prediction Model for User \#97

\begin{tabular}{|c|c|c|c|}
\hline $\begin{array}{c}\text { UserID } 97 \\
\text { (Reds) }\end{array}$ & $\begin{array}{l}\text { 8-colors model } \\
\text { prediction }\end{array}$ & Our prediction model & Actual label \\
\hline Running & -1 & 1 & 1 \\
\hline Walking & -1 & 1 & 1 \\
\hline Yoga & -1 & 1 & -1 \\
\hline Swimming & -1 & 1 & 1 \\
\hline Tennis & 1 & -1 & 1 \\
\hline Hiking & -1 & 1 & 1 \\
\hline Basketball & 1 & 1 & 1 \\
\hline Skating & 1 & -1 & -1 \\
\hline Skiing & 1 & -1 & -1 \\
\hline Soccer & 1 & -1 & 1 \\
\hline Dancing & -1 & 1 & 1 \\
\hline Cycling & -1 & -1 & -1 \\
\hline Pilates & -1 & 1 & 1 \\
\hline \multirow[t]{2}{*}{ Strength training } & -1 & -1 & 1 \\
\hline & $\begin{array}{c}5 \text { hit, } 9 \text { miss. Accuracy } \\
=35.7 \%\end{array}$ & $\begin{array}{c}10 \text { hit, } 4 \text { miss Accuracy } \\
=71.4 \%\end{array}$ & \\
\hline
\end{tabular}


The average accuracy of the 8 -colors model among our participants was $36.7 \%$ with a STDEV of $10.3 \%$, compared to the average accuracy of our prediction model was $70.5 \%$ with a STDEV of $8.7 \%$.

\subsubsection{Result of Pilot Study}

As described above, due to time constraints, we did not conduct another round of formal long-term study with the updated system yet. Instead, we invited five of our participants from the previous study (from the Full group) to try the new version of the system and provide feedback. A casual two-question survey was collected after their seven-day use of the new system. Participants provided their responses on a 7-point Likert scale (from 1 $=$ strongly disagree to $7=$ strongly agree). The statements were:

1) I found the recommendation quality of this new version of the application better than the old one;

2) I prefer using this new version of the application over the old one.

For question 1, the average score was 6.4 with a STDEV of 0.55 . Question 2 resulted in an average score of 6.2 with a STDEV of 0.84 . The sample size was too small for formal statistical analysis but it indicated a visible improvement of the new recommendation system.

\subsubsection{Discussion}

Overall, research phase 4 focused on the optimization of the proposed system. Firstly, a survey is carried out to gather data regarding people's exercise preferences. Then based on the survey results, a feature importance experiment was conducted to find out what 
parameters were more important in predicting users' preference for physical activities. Afterward, the recommendation system was re-designed and implemented based on the prediction model. We compared the proposed model to an existing model on the accuracy in predicting user's PA type preference.

This revised system is a relatively new approach in designing a recommendation system for physical activities. The result shows the feasibility of using the player model for personalizing PA as well as the potential of using machine learning in building the recommendation system for PA. Although the dataset we collected for this study was relatively small, the preliminary result shows considerable effect in optimizing the system.

The comparison between our proposed system and the 8-colors model shows that based on our result, it is not sufficient to generate PA recommendation only based on personality type. In fact, personality type has only limited influence on people's preference toward physical activities, while other parameters such as people's lifestyle, physical conditions, and social connections, may play more important roles in determining people's exercise taste.

Existing player type model, and gamified user type model (e.g. BrainHex, Hexad, etc.) were all built based on personality types, but when it applies to exergame, It can be argued that due to the uniqueness of it, our proposed comprehensive exergame player model, could be considered as a potential alternative to replace the traditional personality-based player models (e.g., the 8-colors model), especially in generating recommendation of PA.

There were certain limitations in this study, which include: 
- The binary classification doesn't show the detailed preference level; a multi-class classification may be used for better accuracy and the ranking of Set A.

- Most of the PA from the binary prediction model with lower accuracy were those with a lower response rate. This is due to the overall difference in popularity of the PA. The overall data was unbalanced and our data set is relatively small.

- For different physical activities, the corresponding rank of the important features varied obviously. To generate better recommendations, we could consider each PA separately regarding parameter selection.

- In this system, we did not look at the distance between PA (item-based recommendation method). With a hybrid recommendation method, we could also consider the distance between PA from different perspectives (cost, calorie consumption, in-door/outdoor activity, aerobic/non-aerobic, group/individual, summer/winter sport, hardness, intensity, flexibility, pleasure, and discipline, etc.) 


\section{Chapter 6: Conclusion}

\subsection{Summary of Findings and Contributions}

This thesis presented a four-phase research that aimed at gradually investigating the potential methods of keeping exergame users motivated and engaged longer in the system. Our research questions were:

RQ1: Is it feasible to use wearable activity trackers for gamification of exercise?

RQ2: Whether game features and their release system can improve engagement overtime or not?

RQ3: Is it feasible to provide users with personalized activity recommendations by using continuous player modeling?

RQ4: Can player modeling and gamification techniques improve user engagement/experience towards fitness activities over time?

RQ5: What parameters should exist in the player model?

RQ6: Which existing models and approaches are effective to recommend specific activities to different users?

RQ7: How the player model could be used to recommend activity?

Research phase 1 proposed the use of wearable devices in exergames, towards which a smartphone exergame was designed and implemented that communicates with wearable devices in real-time and receives user inputs accordingly. Combinations of three different 
exercises and two different types of wearable devices were selected as different gaming modes. We performed a detailed user study to evaluate the usability and outcomes of the system. The results show that the approach is motivating and engaging and that commercial wearable activity trackers can indeed be used for this purpose (RQ1). Users demonstrated high satisfaction towards the application and enjoyed the experience. Factors such as user preferences towards different types of exercise or devices were also investigated.

Research phase 2 extended the exergame (developed in phase 1) with a set of more diverse features to evaluate the motivational impacts and game sustainability of a wearable-based gamified exercise and fitness system. The results of this long-term (70day) user study show that engagement and game-play are highly linked, and the number of game features can impact user motivation towards the gamified exercise system. Moreover, consistent updates (gradual addition of new features) not only resulted in increased usage of the application but also had positive impacts on the actual amount of workout activity (RQ2).

Research phase 3 proposed a system for personalized fitness assistants using gamification and continuous player modeling and reported on a long-term study that investigates the effectiveness of our proposal system. The findings show that it is possible to provide personalized activity recommendations by continuously updating a player model based on activity tracking (RQ3). This study also shows the positive effect of this modeling and gamification on user engagement and overall activity (RQ4). 
Research phase 4 focused on the optimization of the proposed system. A feature importance experiment was conducted to find out what parameters were more important in predicting users' preference for physical activities (RQ5). Afterward, the recommendation system was re-designed and implemented. The proposed model resulted in better accuracy when comparing to an existing model in predicting users' preference toward physical activity types (RQ6-7).

Overall, this thesis proposed a new theoretical model which uses player modeling to promote the motivational effect of the gamified system, as well as to enhance the dynamic/adaptive gameplay experience. It also proposed a new architecture for automated personalized activity recommendation for exergames, whereby a player model for activity recommendation is developed and evaluated through a long-term study. The result shows the feasibility of applying player modeling and recommendation system to exergames, as well as how machine learning could be utilized to optimize the player model and the recommendation system.

\subsection{Directions for Future Research}

In general, this research indicated the feasibility and effectiveness of using different technologies (wearables, player modeling, machine learning, etc.) to promote user's motivation and engagement with exergames. These findings can be used to inform the design of personalized and gamified recommender systems in health and fitness, and potentially other applications.

This research open opportunities for future work especially in the area of exploring more gameplay features, adding personalized storyline and UI, multiplayer system, better 
activity recognition models (to recognize more types of daily activities), and evaluation with a larger and more diverse set of participants. Since one of the major limitations of this study was that our participants were mostly limited to college students, the applications developed during this research can be extended to target a broader group of ages, such as seniors or children.

The design phase of the applications could be optimized by following a more user-centric design process with formal usability studies. Furthermore, the player model built in this research work can be further improved to use or propose standardized formats for player model. This can allow data sharing among applications in the future. Finally, the machine learning process of this work can be further investigated from different perspectives such as performance, efficiency and scalability. 


\section{Appendices}

\section{Appendix A Intrinsic Motivation Inventory:}

Interest/Enjoyment was measured by seven sentences:

1) I enjoyed playing this game very much;

2) This game was fun to play;

3) I thought this was a boring game (reverse coded);

4) This game did not hold my attention at all (reverse coded);

5) I would describe this game as very interesting;

6) I thought this game was quite enjoyable;

7) While I was playing this game, I was thinking about how much I enjoyed it.

Perceived Competence was measured by six sentences:

1) I think I am pretty good at this game;

2) I think I did pretty well at this game, compared to other players;

3) After playing this game for a while, I felt pretty competent;

4) I am satisfied with my performance at this time;

5) I was pretty skilled at this game;

6) This was a game that I couldn't do very well (reverse coded).

Effort/Importance was measured by five sentences:

1) I put a lot of effort into this game;

2) I didn't try very hard to do well at this game (reverse coded);

3) I tried very hard at this game;

4) It was important to me to do well at this game.

5) I didn't put much energy into this game (reverse coded).

Pressure/Tension was measured by five sentences:

1) I did not feel nervous at all while doing this (reverse coded);

2) I felt very tense while doing this activity;

3) I was very relaxed in doing these (reverse coded);

4) I was anxious while working on this task;

5) I felt pressured while doing these.

Value/Usefulness was measured by 7 statements;

1) I believe this game could be of some value to me;

2) I think that playing this game is useful for keeping me active;

3) I think this is important to do because it can motivate me to engage in exercise;

4) I would be willing to lay it again because it has some value to me;

5) I think playing this game could help me to exercise more often;

6) I think playing this game could be beneficial to me;

7) I think this is an important game. 


\section{Appendix B EMIC (European Microsoft Innovation Center) recommender system evaluation measurement:}

Perceived recommendation quality was measured by seven sentences:

1) I like the activities recommended by the system;

2) The recommended activities were well-chosen;

3) The recommended activities fitted my preference;

4) The recommended activities were relevant;

5) The system recommended too many bad activities (reverse coded);

6) I didn't like any of the recommended activities (reverse coded);

7) The activities I selected were "the best among the worst" (reverse coded).

Perceived system effectiveness was measured by six sentences:

1) I would recommend the system to others;

2) The system is useless (reverse coded);

3) The system makes me more aware of my choice options;

4) I make better choices with the system;

5) I can find better items without the help of the system (reverse coded);

6) I can find better items using the recommender system.

General trust in technology was measured by four sentences:

1) Technology never works (reverse coded);

2) I'm less confident when I use technology (reverse coded);

3) The usefulness of technology is highly overrated (reverse coded);

4) Technology may cause harm to people (reverse coded).

System-specific privacy concern was measured by four sentences:

1) I'm afraid the system disclosed private information about me (reverse coded);

2) The system invades my privacy (reverse coded);

3) I feel confident that the system respects my privacy;

4) I'm uncomfortable providing private data to the system (reverse coded). 


\section{Appendix C The 8 color of fitness activity suggestions}

\begin{tabular}{|c|c|}
\hline Blues & Rowing, running, walking, yoga, interval training \\
\hline Golds & Swimming, Pilates, yoga, tennis, one-on-one personal training, hiking \\
\hline Reds & Basketball, tennis, racquetball, in-line skating, Frisbee, mountain biking, soccer, skiing \\
\hline Greens & Hiking, orienteering, backpacking, mountain or road cycling, kayaking, rock climbing, windsurfing \\
\hline Silvers & Tai chi, bicycling or jogging with a group, Zumba, Nia, yoga, group cycling \\
\hline Saffrons & Salsa, belly, ballroom, jazz, folk \\
\hline Whites & Hiking, running, yoga, cardio, gym strength training \\
\hline Purples & Lap swimming, cardio and strength training, running, cycling \\
\hline
\end{tabular}




\section{Appendix D Survey items for research phase 4}

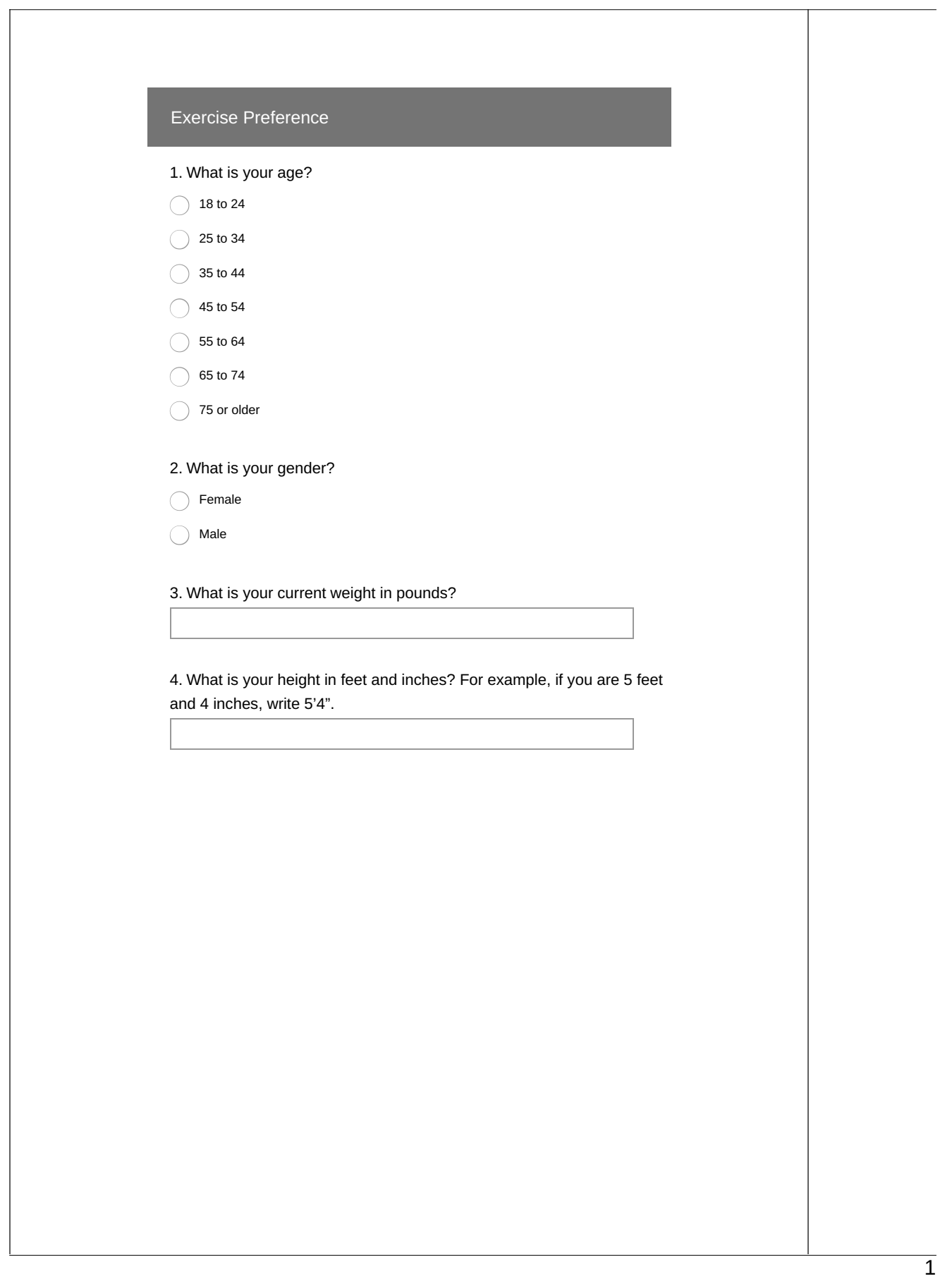




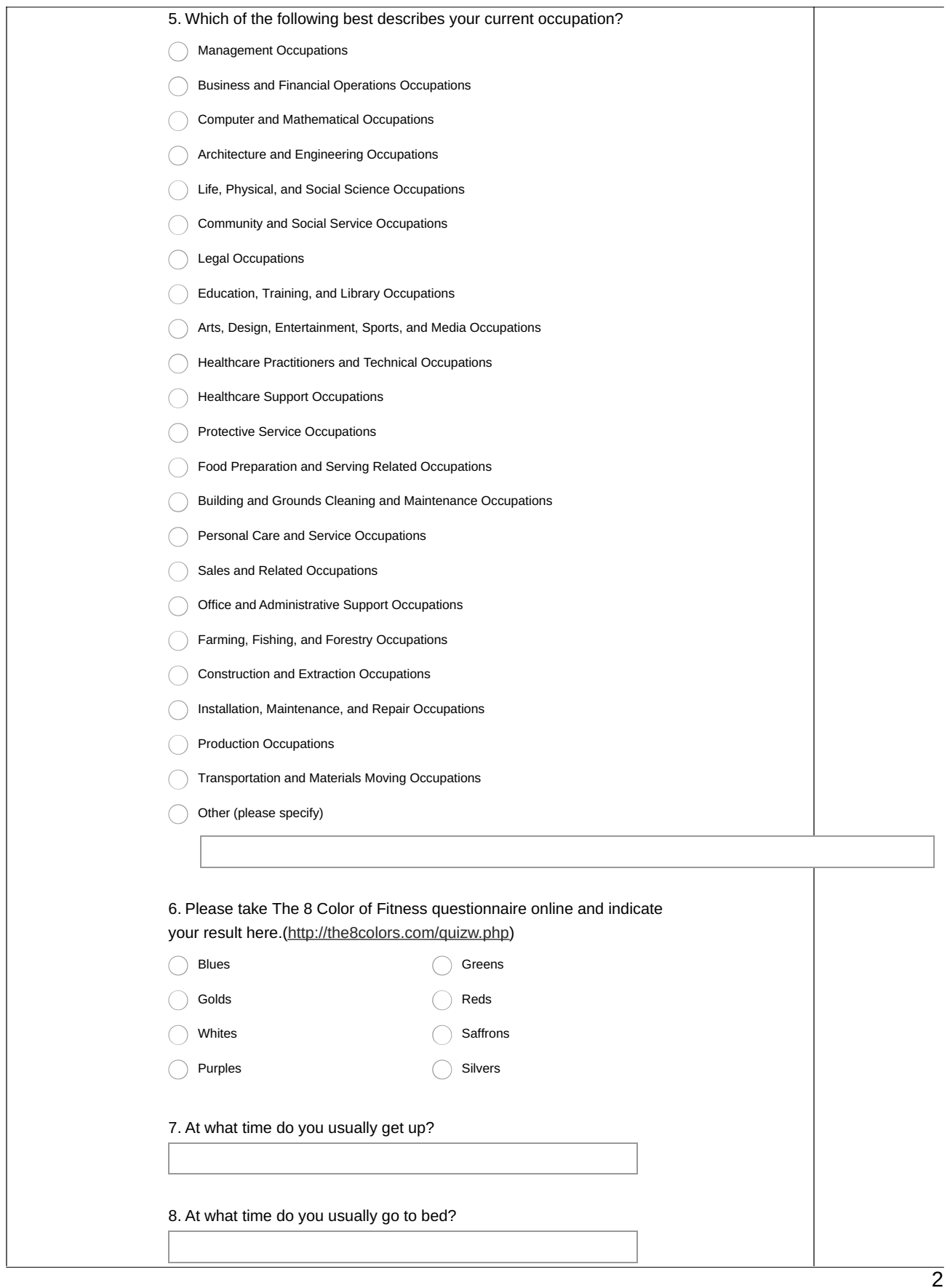




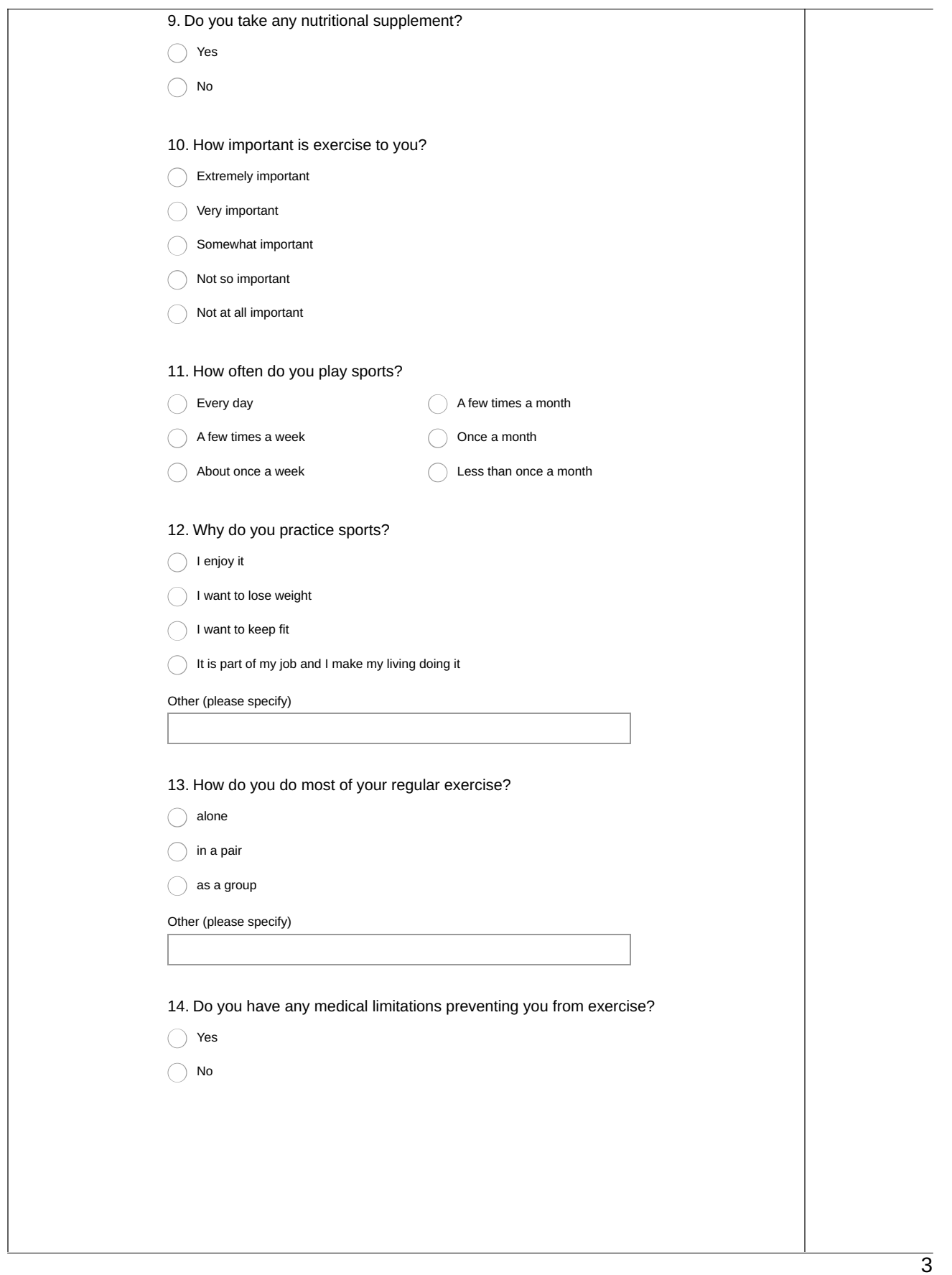




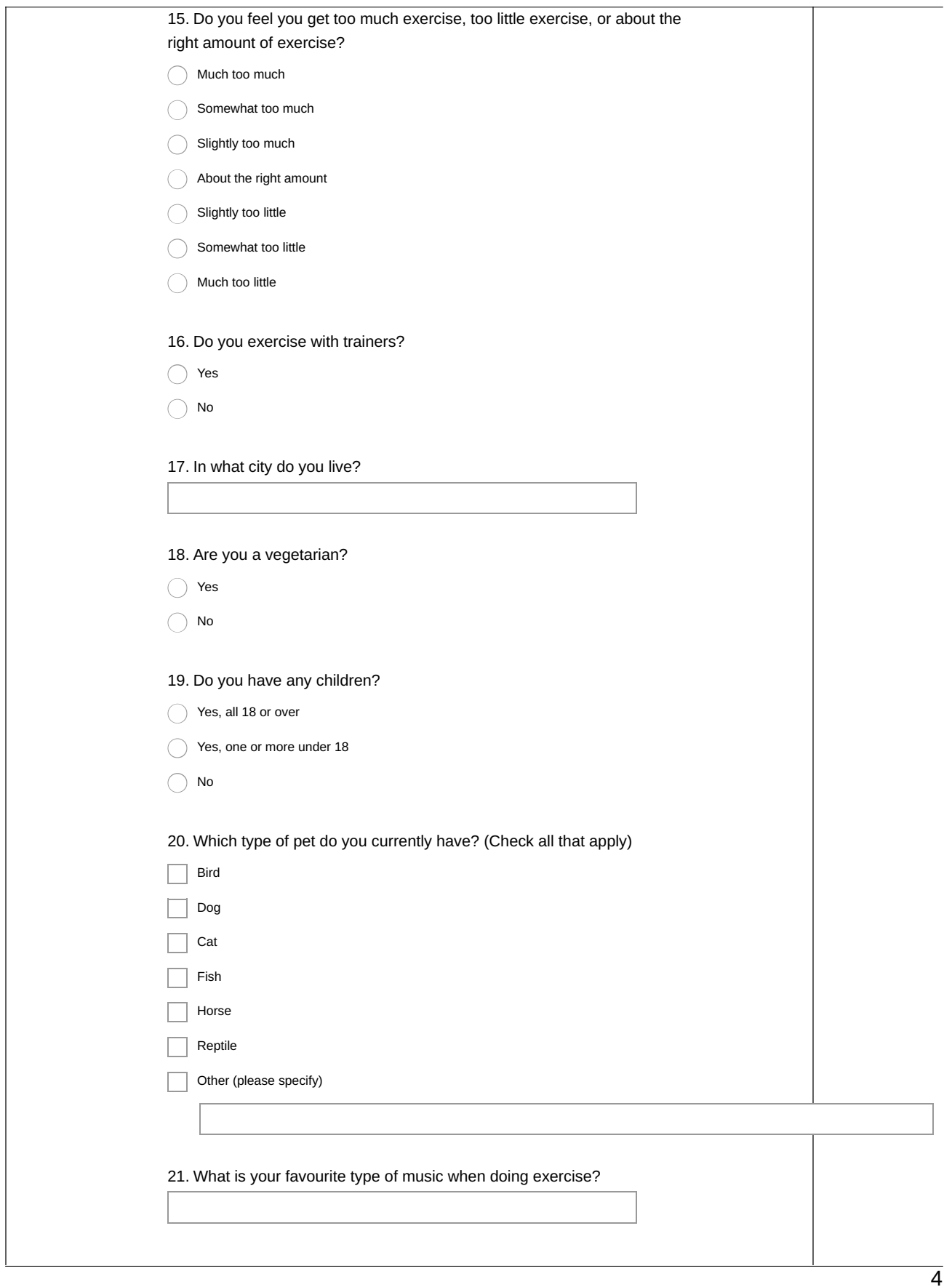




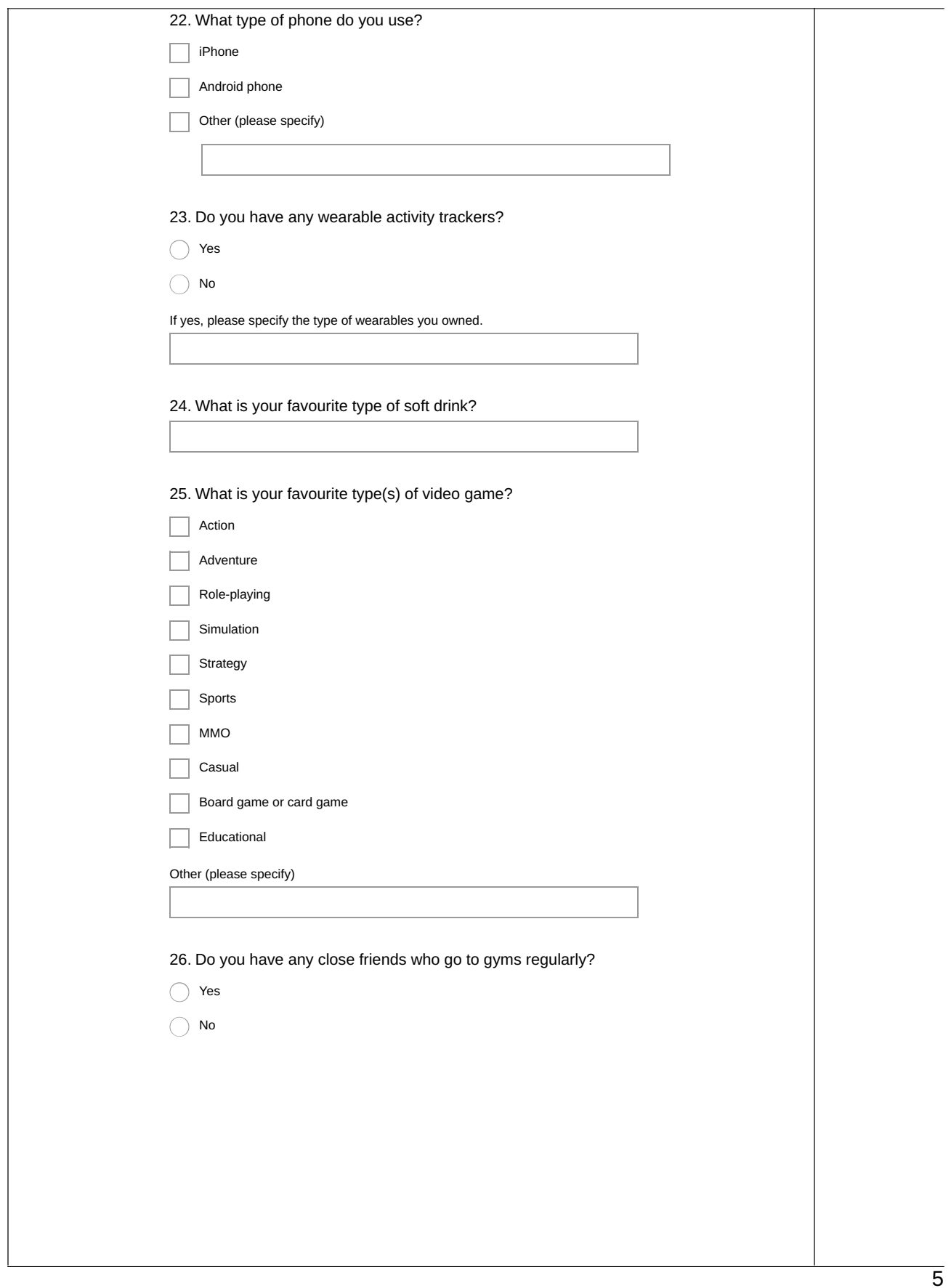

118 


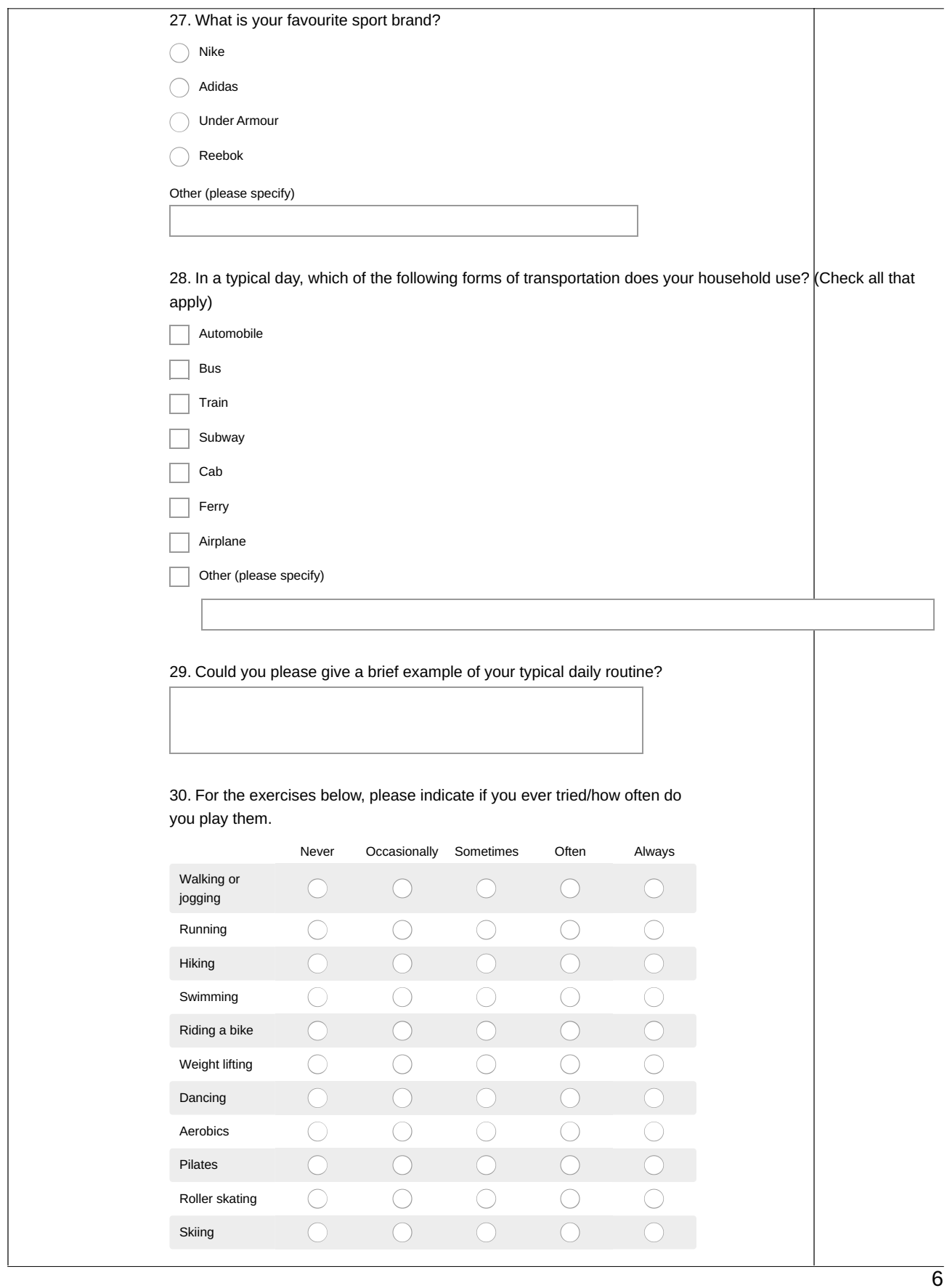




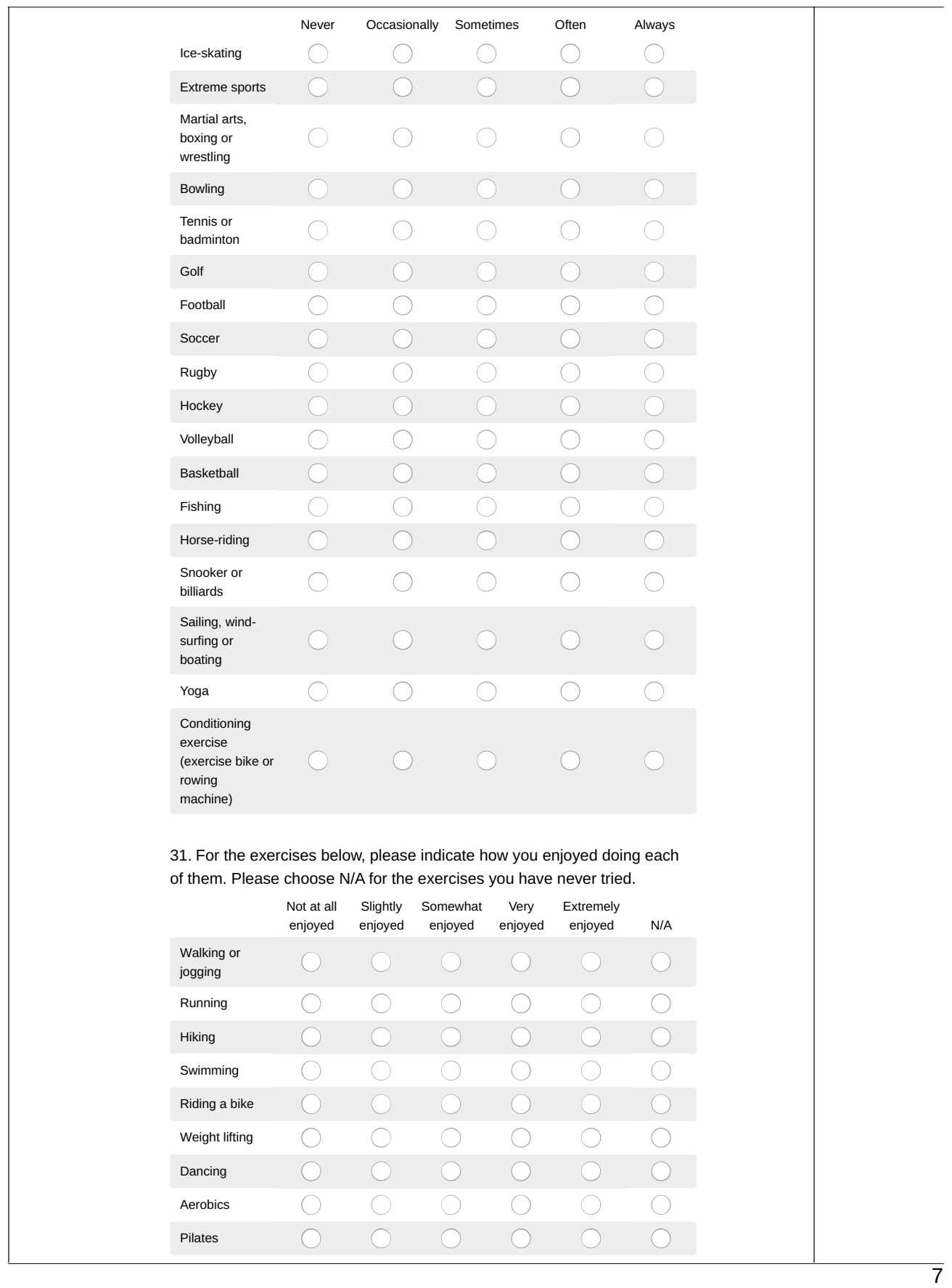




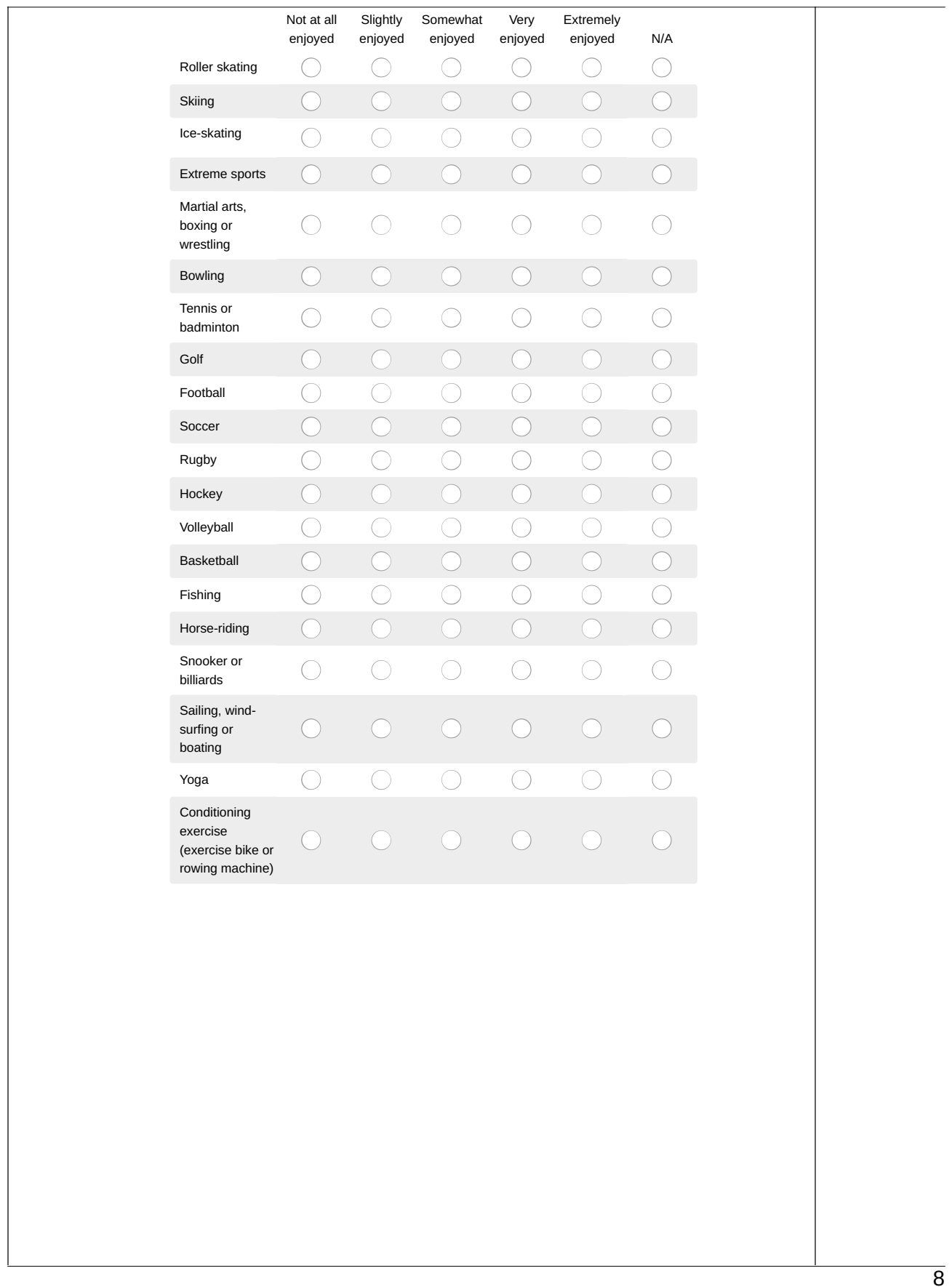




\section{References}

1. (8colors, 2018): 8 Colors Quiz. (2018). Retrieved from http://the8colors.com/quiz.php.

2. (Activityrecognition, 2018): ActivityRecognitionClient | Google APIs for Android | Google Developers. (2018). Retrieved from https://developers.google.com/android/reference/com/google/android/gms/location/ActivityRecognitio nClient.

3. (Aloha, 2013): Ahola, R., Pyky, R., Jämsä, T., Mäntysaari, M., Koskimäki, H., Ikäheimo, T. M., ... \& Korpelainen, R. (2013). Gamified physical activation of young men-a Multidisciplinary PopulationBased Randomized Controlled Trial (MOPO study). BMC public health, 13(1), 32.

4. (Althoff, 2016): Althoff, T., White, R. W., \& Horvitz, E. (2016). Influence of Pokémon Go on physical activity: study and implications. Journal of medical Internet research, 18(12), e315.

5. (Analytics, 2016): Google.com. 2016. Google Analytics - Mobile, Premium and Free Website Analytics - Google. Retrieved September 52016 from https:/www.google.com/analytics

6. (Analytics, 2018): Google Analytics | Google Developers. (2018). Retrieved from https://developers.google.com/analytics/.

7. (Ashe, 2015): Ashe, M. C., Winters, M., Hoppmann, C. A., Dawes, M. G., Gardiner, P. A., Giangregorio, L. M., ... \& Singer, J. (2015). "Not just another walking program": Everyday Activity Supports You (EASY) model — a randomized pilot study for a parallel randomized controlled trial. Pilot and feasibility studies, 1(1), 4.

8. (Bartle, 1996): Bartle, R. (1996). Hearts, clubs, diamonds, spades: Players who suit MUDs. Journal of MUD research, 1(1), 19.

9. (Bateman, 2005): Bateman, C., \& Boon, R. (2005). 21st Century Game Design (Game Development Series). Charles River Media, Inc..

10. (Bateman, 2011): Bateman, C., Lowenhaupt, R., \& Nacke, L. (2011, September). Player typology in theory and practice. In DiGRA Conference.

11. (Bauman, 2006): Bauman, A., Smith, B. J., Maibach, E. W., \& Reger-Nash, B. (2006). Evaluation of mass media campaigns for physical activity. Evaluation and Program Planning, 29(3), 312-322.

12. (Bayne, 2012): Bayne, R. (2012). The Counsellor's Guide to Personality: Understanding Preferences, Motives and Life Stories. Macmillan International Higher Education.

13. (Biddiss, 2010): Biddiss, E., \& Irwin, J. (2010). Active video games to promote physical activity in children and youth: a systematic review. Archives of pediatrics \& adolescent medicine, 164(7), 664672.

14. (Biddle, 2017): Biddle, S. J., Bengoechea, E. G., \& Wiesner, G. (2017). Sedentary behaviour and adiposity in youth: a systematic review of reviews and analysis of causality. International Journal of Behavioral Nutrition and Physical Activity, 14(1), 43.

15. (Blast, 2013): Blast Analytics \& Marketing. 2013. Can You Trust Your Google Analytics Data? $\begin{array}{llll}\text { Retrieved } & \text { September } & 5, & 2016\end{array}$ http://www.blastam.com/blog/can-you-trust-your-google-analytics-data

16. (Broekhuizen, 2010): Broekhuizen, K., van Poppel, M. N., Koppes, L. L., Brug, J., \& van Mechelen, W. (2010). A tailored lifestyle intervention to reduce the cardiovascular disease risk of individuals with Familial Hypercholesterolemia (FH): design of the PRO-FIT randomised controlled trial. BMC Public Health, 10(1), 69.

17. (Brue, 2008): Brue, S (2008). The 8 Colors of Fitness. Oakledge Press, Delray Beach, Florida, USA.

18. (Busch, 2016): Busch, M., Mattheiss, E. E., Hochleitner, W., Hochleitner, C., Lankes, M., Fröhlich, 
P., ... \& Tscheligi, M. (2016). Using Player Type Models for Personalized Game Design-An Empirical Investigation. IxD\&A, 28, 145-163.

19. (Campbell, 2008): Campbell, T., Ngo, B., \& Fogarty, J. (2008, November). Game design principles in everyday fitness applications. In Proceedings of the 2008 ACM conference on Computer supported cooperative work (pp. 249-252). ACM.

20. (Caro, 2018) Caro, K., Feng, Y., Day, T., Freed, E., Fox, B., \& Zhu, J. (2018, May). Understanding the Effect of Existing Positive Relationships on a Social Motion-based Game for Health. In Proceedings of the 12th EAI International Conference on Pervasive Computing Technologies for Healthcare (pp. 7787). ACM.

21. (Chandrashekar, 2014): Chandrashekar, G., \& Sahin, F. (2014). A survey on feature selection methods. Computers \& Electrical Engineering, 40(1), 16-28.

22. (Charles, 2005): Charles, D., Kerr, A., McNeill, M., McAlister, M., Black, M., Kcklich, J., ... \& Stringer, K. (2005, June). Player-centred game design: Player modelling and adaptive digital games. In Proceedings of the digital games research conference (Vol. 285, p. 00100).

23. (Chung, 2016): Chung, A. E., Skinner, A. C., Hasty, S. E., \& Perrin, E. M. (2017). Tweeting to health: a novel mHealth intervention using Fitbits and Twitter to foster healthy lifestyles. Clinical pediatrics, 56(1), 26-32.

24. (Coe, 1992): Coe, C. K. (1992). The MBTI: Potential uses and misuses in personnel administration. Public Personnel Management, 21(4), 511-522.

25. (Consolvo, 2009): Consolvo, S., Klasnja, P., McDonald, D. W., \& Landay, J. A. (2009, April). Goalsetting considerations for persuasive technologies that encourage physical activity. In Proceedings of the 4th international Conference on Persuasive Technology(p. 8). ACM.

26. (Coughlin, 2016): Coughlin, S. S., Whitehead, M., Sheats, J. Q., Mastromonico, J., \& Smith, S. (2016). A review of smartphone applications for promoting physical activity. Jacobs journal of community medicine, 2(1).

27. (Courneya, 1998): Courneya, K. S., \& Hellsten, L. A. M. (1998). Personality correlates of exercise behavior, motives, barriers and preferences: An application of the five-factor model. Personality and Individual differences, 24(5), 625-633.

28. (CPAG, 2018): Canadian Physical Activity Guide. (2018). Retrieved from http://csep.ca/CMFiles/Guidelines/CSEP_PAGuidelines_0-65plus_en.pdf

29. (Drachen, 2009): Drachen, A., Canossa, A., \& Yannakakis, G. N. (2009, September). Player modeling using self-organization in Tomb Raider: Underworld. In Computational Intelligence and Games, 2009. CIG 2009. IEEE Symposium on (pp. 1-8). IEEE.

30. (Evenson, 2015): Evenson, K. R., Goto, M. M., \& Furberg, R. D. (2015). Systematic review of the validity and reliability of consumer-wearable activity trackers. International Journal of Behavioral Nutrition and Physical Activity, 12(1), 159.

31. (Ferro, 2013): Ferro, L. S., Walz, S. P., \& Greuter, S. (2013, September). Towards personalised, gamified systems: an investigation into game design, personality and player typologies. In Proceedings of The 9th Australasian Conference on Interactive Entertainment: Matters of Life and Death (p. 7). ACM.

32. (Fit, 2018): Android APIs | Google Fit | Google Developers. (2018). Retrieved from https://developers.google.com/fit/android/.

33. (Garcin, 2016): Garcin, F., \& Faltings, B. (2016). "Recommender system for an online multimedia content provider.", U.S. Patent Application No. 14/733,304.

34. (Gobel, 2010): Göbel, S., Hardy, S., Wendel, V., Mehm, F., \& Steinmetz, R. (2010, October). Serious games for health: personalized exergames. In Proceedings of the 18th ACM international conference on Multimedia (pp. 1663-1666). ACM.

35. (Guo, 2017): Guo, X., Liu, J., \& Chen, Y. (2017, May). FitCoach: Virtual fitness coach empowered by wearable mobile devices. In INFOCOM 2017-IEEE Conference on Computer Communications, 
IEEE (pp. 1-9). IEEE.

36. (Hamari, 2013): Hamari, J., \& Koivisto, J. (2013, June). Social Motivations To Use Gamification: An Empirical Study Of Gamifying Exercise. In ECIS (Vol. 105).

37. (He, 2014): He, Q., Agu, E., Strong, D., \& Tulu, B. (2014, November). RecFit: a context-aware system for recommending physical activities. In Proceedings of the 1st Workshop on Mobile Medical Applications (pp. 34-39). ACM.

38. (Hexad, 2018): Gamified UK Gamification User Type HEXAD Test. (2018). Retrieved from https://gamified.uk/UserTypeTest2016/user-type-test.php.

39. (Hockeyapp, 2016): Hockeyapp.net. 2016. HockeyApp - The Platform for Your Apps. Retrieved August 5, 2016 from https://www.hockeyapp.net

40. (Hockeyapp, 2018): HockeyApp - The Platform for Your Apps. (2018). Retrieved from https://hockeyapp.net/.

41. (HSC, 2016): Health Status of Canadians. (2016). Retrieved from http://healthycanadians.gc.ca/publications/department-ministere/state-public-health-status-2016-etatsante-publique-statut/alt/pdf-eng.pdf

42. (Imi, 2016): Intrinsic Motivation Inventory. 2016. Self-Determination Theory. Retrieved from http://selfdeterminationtheory.org/intrinsic-motivation-inventory/.

43. (Jaccard, 1984): Jaccard, J., Becker, M. A., \& Wood, G. (1984). Pairwise multiple comparison procedures: A review. Psychological Bulletin, 96(3), 589.

44. (Jakicic, 2005): Jakicic, J. M., \& Otto, A. D. (2005). Physical activity considerations for the treatment and prevention of obesity-. The American journal of clinical nutrition, 82(1), 226S-229S.

45. (John, 1991): John, O. P., Donahue, E., \& Kentle, R. (1991). 'The “Big Five. Inventory-version $4 a$ and, 54 .

46. (Judge, 2002): Judge, T. A., Bono, J. E., Ilies, R., \& Gerhardt, M. W. (2002). Personality and leadership: a qualitative and quantitative review. Journal of applied psychology, 87(4), 765.

47. (Jung, 2009): Jung, Y., Li, K. J., Janissa, N. S., Gladys, W. L. C., \& Lee, K. M. (2009, December). Games for a better life: effects of playing Wii games on the well-being of seniors in a long-term care facility. In Proceedings of the Sixth Australasian Conference on Interactive Entertainment (p. 5). ACM.

48. (Kalton, 2003): Kalton, G., \& Flores-Cervantes, I. (2003). Weighting methods. Journal of Official Statistics, 19(2), 81.

49. (Kassambara, 2017): Kassambara, A., \& Mundt, F. (2017). Package 'factoextra'. Extract and visualize the results of multivariate data analyses, 76.

50. (Knijnenburg, 2012): Knijnenburg, B. P., Willemsen, M. C., Gantner, Z., Soncu, H., \& Newell, C. (2012). Explaining the user experience of recommender systems. User Modeling and User-Adapted Interaction, 22(4-5), 441-504.

51. (Kodinariya, 2013): Kodinariya, T. M., \& Makwana, P. R. (2013). Review on determining number of Cluster in K-Means Clustering. International Journal, 1(6), 90-95.

52. (Lakka, 2003): Lakka, T. A., Laaksonen, D. E., Lakka, H. M., Männikkö, N. I. K. O., Niskanen, L. K., Rauramma, R., \& Salonen, J. T. (2003). Sedentary lifestyle, poor cardiorespiratory fitness, and the metabolic syndrome. Medicine \& Science in Sports \& Exercise.

53. (Lara, 2013): Lara, O. D., \& Labrador, M. A. (2013). A survey on human activity recognition using wearable sensors. IEEE Communications Surveys and Tutorials, 15(3), 1192-1209.

54. (Macvean, 2013): Macvean, A., \& Robertson, J. (2013, April). Understanding exergame users' physical activity, motivation and behavior over time. In Proceedings of the SIGCHI Conference on Human Factors in Computing Systems (pp. 1251-1260). ACM.

55. (Marczewski, 2015): Marczewski, A. (2015). Even ninja monkeys like to play: Gamification, game 
thinking \& motivational design. Gamified UK.

56. (Matallaoui, 2017): Matallaoui, A., Hanner, N., \& Zarnekow, R. (2017). Introduction to gamification: Foundation and underlying theories. In Gamification (pp. 3-18). Springer, Cham.

57. (Mccrae, 1992): McCrae, R. R., \& John, O. P. (1992). An introduction to the five-factor model and its applications. Journal of personality, 60(2), 175-215.

58. (McGonial, 2011): McGonigal, J. (2011). Reality is broken: Why games make us better and how they can change the world. Penguin.

59. (Middelweerd, 2014): Middelweerd, A., Mollee, J. S., van der Wal, C. N., Brug, J., \& Te Velde, S. J. (2014). Apps to promote physical activity among adults: a review and content analysis. International journal of behavioral nutrition and physical activity, 11(1), 97.

60. (Mueller, 2011): Florian 'Floyd' Mueller, Firaz Peer, Stefan Agamanolis, and Jennifer Sheridan. 2011. Gamification and exertion. Proceedings of the Workshop on Gamification at the Conference on Human Factors in Computing Systems. ACM.

61. (Myers, 1977): Carlyn, M. (1977). An assessment of the Myers-Briggs type indicator. Journal of personality assessment, 41(5), 461-473.

62. (Nacke, 2011): Nacke, L. E., Bateman, C., \& Mandryk, R. L. (2011, October). BrainHex: preliminary results from a neurobiological gamer typology survey. In International Conference on Entertainment Computing (pp. 288-293). Springer, Berlin, Heidelberg.

63. (Nacke, 2014): Nacke, L. E., Bateman, C., \& Mandryk, R. L. (2014). BrainHex: A neurobiological gamer typology survey. Entertainment computing, 5(1), 55-62.

64. (Nacke, 2017): Nacke, L.E., and Deterding, S. (2017). Editorial : The maturing of gamification research. Computers in Human Behaviour. 450-454. ISSN 0747-5632

65. (Nicholson, 2015): Nicholson, S. (2015). A recipe for meaningful gamification. In Gamification in education and business (pp. 1-20). Springer International Publishing

66. (Orji, 2013): Orji, R., Mandryk, R. L., Vassileva, J., \& Gerling, K. M. (2013, April). Tailoring persuasive health games to gamer type. In Proceedings of the SIGCHI Conference on Human Factors in Computing Systems (pp. 2467-2476). ACM.

67. (Orji, 2014): Orji, R. (2014). Design for behaviour change: a model-driven approach for tailoring persuasive technologies (Doctoral dissertation, University of Saskatchewan).

68. (Orji, 2017): Orji, R., Mandryk, R. L., \& Vassileva, J. (2017). Improving the efficacy of games for change using personalization models. ACM Transactions on Computer-Human Interaction (TOCHI), 24(5), 32.

69. (Orji, 2018): Orji, R., Tondello, G. F., \& Nacke, L. E. (2018, April). Personalizing persuasive strategies in gameful systems to gamification user types. In Proceedings of the 2018 CHI Conference on Human Factors in Computing Systems (p. 435). ACM.

70. (Pedersen, 2010): Pedersen, C., Togelius, J., \& Yannakakis, G. N. (2010). Modeling player experience for content creation. IEEE Transactions on Computational Intelligence and AI in Games, 2(1), 54-67.

71. (Perron, 2011): Perron, R. M., Graham, C. A., Feldman, J. R., Moffett, R. A., \& Hall, E. E. (2011). Do exergames allow children to achieve physical activity intensity commensurate with national guidelines?. International journal of exercise science, 4(4), 257.

72. (Piwek, 2016): Piwek, L., Ellis, D. A., Andrews, S., \& Joinson, A. (2016). The rise of consumer health wearables: promises and barriers. PLoS Medicine, 13(2), e1001953.

73. (Positivegaming, 2016): Positive Gaming. 2016. The Benefits of Exergaming. Retrieved September 5, 2016 from http://www.positivegaming.com/positivegaming/benefits/exergaming-benefits

74. (Rhodes, 2006): Rhodes, R. E., \& Smith, N. E. I. (2006). Personality correlates of physical activity: a review and meta-analysis. British journal of sports medicine, 40(12), 958-965.

75. (Saelens, 2003): Saelens, B. E., Sallis, J. F., \& Frank, L. D. (2003). Environmental correlates of walking and cycling: findings from the transportation, urban design, and planning literatures. Annals of 
behavioral medicine, 25(2), 80-91.

76. (Anderson, 1985): Anderson, J. R., Boyle, C. F., \& Reiser, B. J. (1985). Intelligent tutoring systems. Science, 228(4698), 456-462.

77. (BBC, 2019):sports A-Z, (2019) retrieved from: https://www.bbc.com/sport/all-sports

78. (Begis, 2000): Begis, G. (2000). U.S. Patent No. 6,106,395. Washington, DC: U.S. Patent and Trademark Office.

79. (Charles, 2004): Charles, D., \& Black, M. (2004, November). Dynamic player modeling: A framework for player-centered digital games. In Proc. of the International Conference on Computer Games: Artificial Intelligence, Design and Education (pp. 29-35).

80. (Edwards, 2016): Edwards, E. A., Lumsden, J., Rivas, C., Steed, L., Edwards, L. A., Thiyagarajan, A., ... \& Taylor, S. (2016). Gamification for health promotion: systematic review of behaviour change techniques in smartphone apps. BMJ open, 6(10), e012447.

81. (Hunicke, 2005): Hunicke, R. (2005, June). The case for dynamic difficulty adjustment in games. In Proceedings of the 2005 ACM SIGCHI International Conference on Advances in computer entertainment technology (pp. 429-433). ACM.

82. (Johnson, 2016): Johnson, D., Deterding, S., Kuhn, K. A., Staneva, A., Stoyanov, S., \& Hides, L. (2016). Gamification for health and wellbeing: A systematic review of the literature. Internet interventions, 6, 89-106.

83. (Lister, 2014): Lister, C., West, J. H., Cannon, B., Sax, T., \& Brodegard, D. (2014). Just a fad? Gamification in health and fitness apps. JMIR serious games, 2(2), e9.

84. (Rabbi, 2015): Rabbi, M., Aung, M. H., Zhang, M., \& Choudhury, T. (2015, September). MyBehavior: automatic personalized health feedback from user behaviors and preferences using smartphones. In Proceedings of the 2015 ACM International Joint Conference on Pervasive and Ubiquitous Computing (pp. 707-718). ACM.

85. (Sami, 2008): Sami, A., Nagatomi, R., Terabe, M., \& Hashimoto, K. (2008, July). Design of Physical Activity Recommendation System. In IADIS European Conf. Data Mining (pp. 148-152).

86. (Schwenk, 2014): Schwenk, M., Grewal, G. S., Honarvar, B., Schwenk, S., Mohler, J., Khalsa, D. S., \& Najafi, B. (2014). Interactive balance training integrating sensor-based visual feedback of movement performance: a pilot study in older adults. Journal of neuroengineering and rehabilitation, 11(1), 164.

87. (Sinclair, 2007): Sinclair, J., Hingston, P., \& Masek, M. (2007, December). Considerations for the design of exergames. In Proceedings of the 5th international conference on Computer graphics and interactive techniques in Australia and Southeast Asia (pp. 289-295). ACM.

88. (Smeddinck, 2015): Smeddinck, J. D., Herrlich, M., \& Malaka, R. (2015, April). Exergames for physiotherapy and rehabilitation: a medium-term situated study of motivational aspects and impact on functional reach. In Proceedings of the 33rd Annual ACM Conference on Human Factors in Computing Systems (pp. 4143-4146). ACM.

89. (Sullivan, 1994): Sullivan, J., Sullivan, J. W., \& Tyler, S. W. (1994). Intelligent user interfaces.

90. (Tavakol, 2011): Tavakol, M., \& Dennick, R. (2011). Making sense of Cronbach's alpha. International journal of medical education, 2, 53.

91. (Taylor, 2017): Taylor, K. (2017). The Relationship Between Personality Types and Exercise Preferences and Behaviors.

92. (Tondello, 2016): Tondello, G. F., Wehbe, R. R., Diamond, L., Busch, M., Marczewski, A., \& Nacke, L. E. (2016, October). The gamification user types hexad scale. In Proceedings of the 2016 annual symposium on computer-human interaction in play (pp. 229-243). ACM.

93. (Toscos, 2011): Toscos, T., Consolvo, S., McDonald, D.W. (2011) Barriers to Physical Activity: A Study of Self-revelation in an Online Community. Journal of Medical Systems, 35(5): $1225-1242$. 
94. (Tregillus, 2016): Tregillus, S., \& Folmer, E. (2016, May). Vr-step: Walking-in-place using inertial sensing for hands free navigation in mobile vr environments. In Proceedings of the $2016 \mathrm{CHI}$ Conference on Human Factors in Computing Systems (pp. 1250-1255). ACM.

95. (Tremblay, 2010) Tremblay, M. S., Colley, R. C., Saunders, T. J., Healy, G. N., \& Owen, N. (2010). Physiological and health implications of a sedentary lifestyle. Applied physiology, nutrition, and metabolism, 35(6), 725-740.

96. (Vaughan, 2016): Vaughan, N., Gabrys, B., \& Dubey, V. N. (2016). An overview of self-adaptive technologies within virtual reality training. Computer Science Review, 22, 65-87.

97. (Wagstaffm 2001): Wagstaff, K., Cardie, C., Rogers, S., \& Schrödl, S. (2001, June). Constrained kmeans clustering with background knowledge. In Icml (Vol. 1, pp. 577-584).

98. (Wang, 2006): Wang, J., De Vries, A. P., \& Reinders, M. J. (2006, August). Unifying user-based and item-based collaborative filtering approaches by similarity fusion. In Proceedings of the 29th annual international ACM SIGIR conference on Research and development in information retrieval (pp. 501508). ACM.

99. (Wang, 2015): Wang, J. B., Cadmus-Bertram, L. A., Natarajan, L., White, M. M., Madanat, H., Nichols, J. F., ... \& Pierce, J. P. (2015). Wearable sensor/device (Fitbit One) and SMS text-messaging prompts to increase physical activity in overweight and obese adults: a randomized controlled trial. Telemedicine and e-Health, 21(10), 782-792.

100.(Washington, 2014): Washington, W. D., Banna, K. M., \& Gibson, A. L. (2014). Preliminary efficacy of prize-based contingency management to increase activity levels in healthy adults. Journal of Applied Behavior Analysis, 47(2), 231-245.

101.(Weather, 2018): API | Weather Underground. (2018). Retrieved from https://www.wunderground.com/weather/api/.

102.(Whitehead, 2010): Whitehead, A., Johnston, H., Nixon, N., \& Welch, J. (2010, July). Exergame effectiveness: what the numbers can tell us. In Proceedings of the 5th ACM SIGGRAPH Symposium on Video Games (pp. 55-62). ACM.

103.(Who, 2010): World Health Organization (2010). Global Recommendations on Physical Activity for Health. World Health Organization.

104.(Wiggins, 1992): Wiggins, J. S., \& Pincus, A. L. (1992). Personality: Structure and assessment. Annual review of psychology, 43(1), 473-504.

105.(Wilmot, 2012): Wilmot, E. G., Edwardson, C. L., Achana, F. A., Davies, M. J., Gorely, T., Gray, L. J., ... \& Biddle, S. J. (2012). Sedentary time in adults and the association with diabetes, cardiovascular disease and death: systematic review and meta-analysis.

106.(Wit, 2018): Wit.ai. (2018). Retrieved from https://wit.ai/.

107.(Wylie, 2010): Wylie, J. 2010. Fitness gamification: concepts, characteristics, and applications. Retrieved from: http://www.justintwylie.com/wp-content/uploads/2014/12/JUSTIN-WYLIE-FitnessGamification.pdf

108.(Xu, 2012): Xu, Y., Poole, E. S., Miller, A. D., Eiriksdottir, E., Kestranek, D., Catrambone, R., \& Mynatt, E. D. (2012, February). This is not a one-horse race: understanding player types in multiplayer pervasive health games for youth. In Proceedings of the ACM 2012 conference on computer supported cooperative work(pp. 843-852). ACM.

109.(Yannakakis, 2013): Yannakakis, G. N., Spronck, P., Loiacono, D., \& André, E. (2013). Player modeling. In Dagstuhl Follow-Ups (Vol. 6). Schloss Dagstuhl-Leibniz-Zentrum fuer Informatik.

110.(Yee, 2006): Yee, N. (2006). Motivations for play in online games. CyberPsychology \& behavior, 9(6), 772-775.

111.(Zhao, 2016): Zhao, Z., Etemad, S. A., \& Arya, A. (2016). Gamification of exercise and fitness using wearable activity trackers. In Proceedings of the 10th International Symposium on Computer Science in Sports (ISCSS) (pp. 233-240). Springer, Cham. 
112.(Zhao, 2017): Zhao, Z., Arya, A., Whitehead, A., Chan, G., \& Etemad, S. A. (2017, May). Keeping Users Engaged through Feature Updates: A Long-Term Study of Using Wearable-Based Exergames. In $C H I$ (pp. 1053-1064).

113.(Zhao, 2018): Zhao, Z., Arya, A., Orji, R., \& Chan, G., "Long-term Study of a Personalized Fitness Recommender System using Gamification and Continuous Player Modeling", under revision for $A C M$ Transaction on CHI. 